
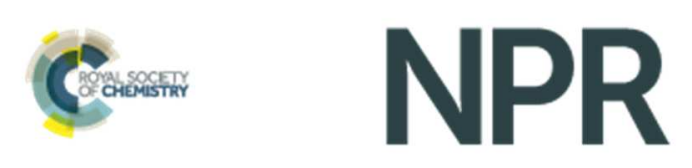

\title{
Recent advances and perspectives in the design and development of polymyxins
}

\begin{tabular}{|r|l|}
\hline Journal: & Natural Product Reports \\
\hline Manuscript ID & NP-REV-04-2017-000023.R1 \\
\hline Article Type: & Review Article \\
\hline Date Submitted by the Author: & n/a \\
\hline Complete List of Authors: & $\begin{array}{l}\text { Rabanal, Francesc; Universitat de Barcelona Facultat de Quimica, Organic } \\
\text { Chemistry Section, Dept of Inorganic and Organic Chemistry } \\
\text { Cajal, Yolanda; University of Barcelona, Department of Pharmaceutical } \\
\text { Technology and Physical Chemistry }\end{array}$ \\
\hline
\end{tabular}




\title{
Natural Products Reports
}

\section{REVIEW}

Received 00th January 20xx, Accepted 00th January 20xx

DOI: $10.1039 / \times 0 \times x 00000 x$

www.rsc.org/

\section{Recent advances and perspectives in the design and development of polymyxins}

\author{
Francesc Rabanal $^{\mathrm{a}^{*}}$ and Yolanda Cajal ${ }^{\mathrm{b}}$ \\ Covering: 1947-early 2017, particularly from 2005-early 2017
}

The rise of bacterial pathogens with acquired resistance to almost all available antibiotics is becoming a serious public health issue. Polymyxins, antibiotics that were mostly abandoned a few decades ago because of toxicity concerns, are ultimately considered as a last-line therapy to treat infections caused by multi-drug resistant Gram-negative bacteria. This review surveys the progress in understanding polymyxin structure, chemistry, mechanisms of antibacterial activity and nephrotoxicity, biomarkers, synergy and combination with other antimicrobial agents and antibiofilm properties. An update of recent efforts in the design and development of a new generation of polymyxin drugs is also discussed. A novel approach considering the modification of the scaffold of polymyxins to integrate metabolism and detoxification issues into the drug design process is a promising new line to potentially prevent accumulation in kidney and reduce nephrotoxicity.

1. Introduction

2. Naturally ocurring polymyxins

3. Antimicrobial profile

4. Commercially available polymyxins

5. Mechanism of action

6. Resistance to polymyxins

7. Combination with other antibiotics

8. Synergy with antifungals

9. Antibiofilm activity

10. Toxicity

10.1. Mechanism of nephrotoxicity

10.2 Biomarkers of nephrotoxicity

11. Design and development of new polymyxins

11.1 Synthetic preparation of polymyxin analogs

11.2 CB-182,804 analog

11.3 Pfizer 5x analog

11.4 Monash FADDI analogs

11.5 Queensland analogs

11.6 Northern Antibiotics analogs

11.7 Cantab analogs

11.8 Scaffold modified polymyxins

11.9 MicuRx analogs

11.10 Barcelona analogs

12. Conclusions and perspectives

13. Acknowledgements

14. References

\footnotetext{
a. Organic Chemistry Section, Department of Inorganic and Organic Chemistry, Faculty of Chemistry, University of Barcelona. E-mail: frabanal@ub.edu ${ }^{b}$ Department of Pharmacy, Pharmaceutical Technology and Physical Chemistry, Faculty of Pharmacy and Food Sciences, University of Barcelona. E-mail: ycajal@ub.edu ${ }^{b}$ Institute of Nanoscience and Nanotechnology (IN2UB), University of Barcelona. † Footnotes relating to the title and/or authors should appear here. Electronic
}

\section{Introduction}

Polymyxins are a group of antimicrobial cyclic lipopeptides discovered in $1947 . .^{1-3}$ They are produced by fermentation of strains of Paenibacillus polymyxa (formerly known as Bacillus polymyxa). Polymyxins consist of a heterogeneous mixture composed of up to 30 closely related lipopeptides (Table I). ${ }^{4-7}$ The term "polymyxin" is accepted as the general name for this class of antibiotics produced by $P$. polymyxa. Polymyxin B and colistin (polymyxin E) are the most known members of this family as they were commonly used as antibiotics in hospitals from late 1950s to late 1970s, approximately. Then, they were gradually withdrawn from the clinical practice due to toxicity issues such as adverse neurological effects and most importantly, nephrotoxicity concerns. In addition, novel aminoglycosides (gentamicin) and second- and thirdgeneration cephalosporins showing less toxic side effects became available. ${ }^{8}$ However, the emergence of Gram-negative bacteria that are resistant to almost all classes of available antibiotics has resulted in the rescue of polymyxins as a last resort for patients whose other treatment options were limited.

Antibiotic resistance is becoming a serious public health issue. In the USA, for instance, at least 2 million people are infected by antibiotic-resistant bacteria and at least 23,000 people die each year as a direct consequence. ${ }^{9,10}$ A similar situation is taking place in Europe. ${ }^{11,12}$ The WHO has recently issued a list of the most critical pathogenic bacteria for which new antibiotics are urgently needed: carbapenem-resistant strains of Acinetobacter baumannii, Pseudomonas aeruginosa, and Enterobacteriaceae. ${ }^{13}$

Polymyxin B and colistin are now used as a last-line therapy to treat infections caused by multi-drug resistant bacteria such as $P$. aeruginosa, A. baumannii, Klebsiella pneumoniae, and Escherichia coli . These bacteria are part of the so-called ESKAPE bacteria, thus nicknamed by the Infectious Disease Society of America that 
Table 1: Structure of naturally occurring polymyxins $B$ and $E$ (colistin), clinically relevant members of the polymyxin family§

\begin{tabular}{|l|l|l|l|}
\hline Polymyxin & Fatty acyl tail & Aa 6 & Aa7 \\
\hline B1 & (S)-6-methyloctanoyl & D-Phe & Leu \\
\hline B1-Ile & (S)-6-methyloctanoyl & D-Phe & Ile \\
\hline B2 & 6-methyheptanoyl & D-Phe & Leu \\
\hline B3 & octanoyl & D-Phe & Leu \\
\hline B4 & heptanoyl & D-Phe & Leu \\
\hline B5 & nonanoyl & D-Phe & Leu \\
\hline B6 & 3-hydroxy-6-methyloctanoyl & D-Phe & Leu \\
\hline E1 & (S)-6-methyloctanoyl & D-Leu & Leu \\
\hline E2 & 6-methyheptanoyl & D-Leu & Leu \\
\hline E3 & octanoyl & D-Leu & Leu \\
\hline E4 & heptanoyl & D-Leu & Leu \\
\hline E7 & 7-methyloctanoyl & D-Leu & Leu \\
\hline E1-Ile & (S)-6-methyloctanoyl & D-Leu & Ile \\
(circulin A) & & & \\
\hline E1-Val & (S)-6-methyloctanoyl & D-Leu & Val \\
\hline E1-Nva & (S)-6-methyloctanoyl & D-Leu & Nva \\
\hline E2-Val & 6-methyheptanoyl & D-Leu & Val \\
\hline E2-Ile & 6-methyheptanoyl & D-Leu & Ile \\
\hline E8-Ile & 7-methylnonanoyl & D-Leu & Ile \\
\hline
\end{tabular}

Table 2: Structure of additional naturally occurring polymyxins $\S$

\begin{tabular}{|l|l|l|l|l|l|}
\hline Polymyxin & Fatty acyl tail & Aa3 & Aa6 & Aa7 & Aa10 \\
\hline A1 & 6-methyloctanoyl & D-Dab & D-Leu & Thr & Thr \\
\hline A2 & 6-methylheptanoyl & D-Dab & D-Leu & Thr & Thr \\
\hline C1 & 6-methyloctanoyl & Dab & Phe & Thr & Thr \\
\hline C2 & 6-methylheptanoyl & Dab & Phe & Thr & Thr \\
\hline D1 & 6-methyloctanoyl & D-Ser & D-Leu & Thr & Thr \\
\hline D2 & 6-methylheptanoyl & D-Ser & D-Leu & Thr & Thr \\
\hline F1 & 6-methyloctanoyl & \multicolumn{2}{|c|}{ (Dab x5, Thr, Leu x2, Ser, Ile) } \\
\hline F2 & 6-methylheptanoyl & \multicolumn{2}{|c|}{ (Dab x5, Thr, Leu x2, Ser, Ile) } \\
\hline M1 & 6-methyloctanoyl & Dab & D-Leu & Thr & Thr \\
\hline M2 & 6-methylheptanoyl & Dab & D-Leu & Thr & Thr \\
\hline S1 & 6-methyloctanoyl & D-Ser & D-Phe & Thr & Thr \\
\hline T1 & 6-methyloctanoyl & Dab & D-Phe & Leu & Leu \\
\hline T2 & 6-methylheptanoyl & Dab & D-Phe & Leu & Leu \\
\hline PMB1 & 6-methyloctanoyl & D-Dab & D-Phe & Thr & Thr \\
\hline PMB2 & 6-methylheptanoyl & D-Dab & D-Phe & Thr & Thr \\
\hline P1 & 6-methyloctanoyl & D-Dab & D-Phe & Thr & Thr \\
\hline P2 & 6-methylheptanoyl & D-Dab & D-Phe & Thr & Thr \\
\hline
\end{tabular}

$\S$ The reader may find nomenclatures such as "iso" and "ante-iso" fatty acids in the literature. For instance, isooctanoic acid, $\mathrm{i}-\mathrm{C}_{8}$ corresponds to 6-methylheptanoic acid. Anteisononanoic acid, a- $C_{9}$, corresponds to 6 methyloctanoic acid and may appear in two different configurations, $R$ or $S$, since a stereocenter is present in carbon 6 . The configuration of this stereocenter is expected to be $S$ as the branch-chain fatty acid synthesizing system starts in the case of anteiso-fatty acids from the $\alpha$-ketoacid of isoleucine as primer. Isoleucine has an $S$ configuration in carbon 3 , in its sidechain.

has proposed to pursue a global commitment to develop 10 new antibacterial drugs by 2020 (the 10 x '20 Initiative). "ESKAPE" stands for the initials of the above mentioned Gram-negative bacteria together with Gram-positive Enterococcus faecium and Staphylococcus aureus. ${ }^{9}$

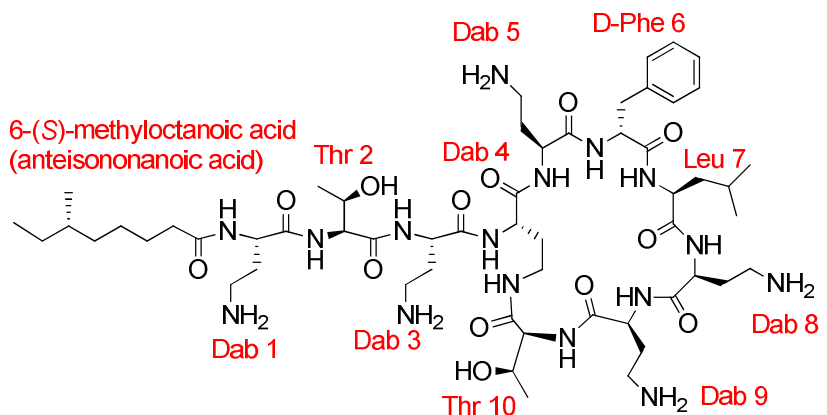

Figure 1: Polymyxin B1 as an example of the general structure of polymyxins. Amino acid positions are numbered from 1 to 10 .

The objective of this review is to gather and analyze the background in the field of polymyxins, highlighting the efforts and new approaches carried out by different groups worldwide toward the design and development of new polymyxin-based compounds potentially capable of overcoming the current drawbacks of the natural compound, particularly, nephrotoxicity. Other fundamental reviews in the field of polymyxins have been published in the last years. As the present manuscript will mainly but not only concentrate in the last decade achievements and new approaches, the reader is also addressed to reviews by Vaara, Velkov\&Li, Brown\&Dawson for a previous background in the area. ${ }^{14-17}$

\section{Naturally occuring polymyxins}

The Paenibacillus genus (previously included in the genus Bacillus) comprises tenths of species that are facultative anaerobic and endospore-forming bacteria. In particular, strains of Paenibacillus polymyxa thrive in the plant rizhosphere, are capable of fixing nitrogen, suppress some plant diseases and promote a healthy growth in plants, such as crops and trees. Hence, $P$. polymyxa strains are used as an effective alternative to the chemical control against a wide set of plant pathogenic fungi and bacteria. Polymyxins, including colistin and circulin, are the main class of peptide antibiotics produced by most strains of $P$. polymyxa although other compounds are produced as well. Other strains produce peptides such as polyxins, polypeptins or fuaricidins. ${ }^{18}$

The general structure of polymyxins consists of a cyclic heptapeptide unit (amino acids 4-10) and a lipotripeptide that bifurcates from the fourth amino acid of the sequence (Figure 1). The lipid unit capping the $\mathrm{N}$-terminal amino acid is a linear or branched fatty acyl moiety, that together with amino acids in the 6th and 7th position define the hydrophobic features of the molecule. The rest of amino acid residues are polar (L-threonines) and amino-containing basic residues (2,4-diaminobutanoic acid) that provide polymyxins with its polycationic nature at physiological $\mathrm{pH}$. Polymyxins are secondary metabolites generated by nonribosomal peptide synthetase enzyme complexes. Hence, they contain non-proteinogenic amino acids as well (not present in regular coded proteins) such as the above mentioned 2,4diaminobutanoic acid, D-phenylalanine or D-Leucine. 
The first polymyxins discovered were reported almost simultaneously by three different teams in 1947: Benedict and Langlykke, ${ }^{1}$, Stansly and coworkers ${ }^{2}$ and Ainsworth and coworkers. ${ }^{3}$ Ainsworth called the antibiotic "aerosporin" since it was obtained from Bacillus aerosporus which later was found to be $B$. polymyxa, essentially the same studied by Benedict and Langlykke. The antimicrobial "aerosporin" is now known as polymyxin A, whereas the so-called "polymyxin" described by Stansly corresponds to polymyxin D. Further investigations by Brownlee and Bushby managed to isolate a third type of polymyxin, which was named polymyxin $B$, and later, polymyxins $C$ and $E$ were found. Polymyxins $A, B, C, D$ and $E$ showed similar antibacterial activity, but an in vivo assay of nephrotoxicity in Wistar rats by estimating the total protein excreted in the urine indicated a striking increase of proteinuria for polymyxins $A, C$ and $D$ but not for polymyxins $B$ and $E .^{19}$

In parallel, Koyama described in 1950 the isolation of an antibiotic from a culture broth of a new strain they named Bacillus polymyxa var. colistinus (Aerosporus colistinus). ${ }^{20}$ The new compound was called colistin, and chemical determination proved it to be cyclic and share an amino acid composition qualitatively identical to that of polymyxin E described by Brownlee. ${ }^{21}$

In 1948, Tetrault and coworkers isolated another antibiotic peptide that named circulin as it was produced by Bacillus circulans. It was found to share a similar composition to the one of polymyxins known so far. It was also active against Gram-negative bacteria. In the following years, other members of the family were discovered, such as polymyxin $M$ found in the soil of Moscow by Khokhlov and coworkers. ${ }^{21,22}$ More recently, Martin and coworkers have isolated mattacin, a cyclic lipodecapeptide produced by Paenibacillus kobensis $\mathrm{M}$ and found by structure elucidation to be identical to polymyxin $\mathrm{M}^{23}$

Polymyxin $\mathrm{P}$, described for the first time in 1969, has been recently found to be the main compound produced by $P$. polymyxa M-1. Polymyxin $P$ suppressed the growth of phytopathogenic Enterobacteriaceae bacteria Erwinia amylovora Ea 273, and E. carotovora, the causative agents of fire blight (in apples and pears) and soft rot, respectively. Hence, it has been proposed as an alternative of chemical bactericides to control these and other plant diseases caused by Gram-negative bacteria. ${ }^{24,25}$

Other polymyxins, such as polymyxin S1 and T1 were isolated from P. polymyxa Rs-6 and E-12, respectively. Polymyxin T1 was found to be active not only against Gram-negative bacteria but also against Gram-positive bacteria, a characteristic shared with polymyxin M. ${ }^{25-29}$ Polymyxin F, produced by Bacillus circulans ATCC 31228 , has also been described. ${ }^{30}$ Similarly, polymyxin $C$ has also been reported (see above) but apparently, has not been subjected to detailed structural studies. ${ }^{28,31,32}$

The last members of the polymyxin family described so far were diasteromers of polymyxin B, named PMB1 and PMB2. ${ }^{33}$ They were produced by $P$. polymyxa PKB-1 and had a D-Dab amino acid in position 3 rather than the usual L-Dab. Their sequences were elucidated by high-resolution mass spectrometry, MS/MS sequencing, and the stereochemistry, by chiral gas chromatography.

Finally, it is worth mentioning a family of natural products called octapeptins, first reported in the mid 1970's. ${ }^{22}$ They are closely related to polymyxins as both families share a similar structure. They consist of a cyclic polycationic peptide sequence, containing a high percentage of 2,4-diaminobutanoic acid and a fatty acyl tail bound through an amide linkage. However, octapeptins contain eight amino acid residues as it may be deduced from its name, with a single exocyclic amino acid stemming from the cycloheptapeptide moiety. A review on octapeptins has recently been published in this journal so the reader is kindly referred to this document for further information. $^{34}$

\section{Antimicrobial profile}

Polymyxins are narrow-spectrum antibiotics since they are only active against Gram-negative bacteria including multi-drug resistant (MDR) strains. This includes some non-fermenting bacteria such as $P$. aeruginosa and Acinetobacter spp. and some members of the Enterobacteriaceae family, Escherichia spp, Klebsiella spp, Enterobacter spp, Citrobacter spp, Salmonella spp, Shigella spp and Haemophilus spp, and Pasteurella spp. However, Proteus spp, Burkholderia spp, Serratia spp (i. e. Serratia marcescens) and Moraxella spp (i. e. Moraxella catarrhalis) and genera Brucella, Neisseria, Chromobacterium and Providencia, have intrinsic resistance to polymyxins. ${ }^{35}$ Finally, it has been reported that polymyxin $E$ was found to be active against some mycobacterial species, e.g. Mycobacterium xenopi, $M$. intracellulare, $M$. tuberculosis, $M$. fortuitum, M. phlei and $M$. smegmatis. ${ }^{36,37}$ Polymyxin B also exhibited activity against Cryptococcus neoformans fungus. ${ }^{38}$

\section{Commercially available polymyxins}

Since polymyxins are manufactured by fermentation procedures, they have a heterogeneous composition. They contain several structurally related components such as isomers and homologous compounds (Table 1). For instance, the major constituents of polymyxin $B$ obtained from $P$. polymyxa are the related polymyxins B1, B2, B3 and B1-lle, differing only in the fatty acyl moiety and the amino acid in position 7 (Leu or lle). Their composition and antibacterial activity have been recently studied in detail. A typical proportion of components in polymyxin B would be $c a 70-74 \%$ of the B1 type, $13-16 \%$ of B2, 3-5\% of B3 and around 8-9 \% of lle$B 1 .^{39,40}$ The activity of some of these components have been assessed individually in strains of $P$. aeruginosa, A. baumannii and $K$. pneumoniae including multi-drug resistant isolates. The results of antimicrobial activity measured by means of their MIC (minimal inhibitory concentration) showed differences within the variability generally accepted for such a kind of assays. Apparently, the minor structural differences (length of the fatty acid tail and compound isomers) among the components did not affect much their in vitro potency. ${ }^{40,41}$ Nevertheless, it is worth mentioning that one of the minor components, polymyxin B3, showed higher activity than the rest against $P$. aeruginosa, $E$. coli and $K$. pneumoniae 
while the compound B1-lle was more potent against $A$. baumannii. In the same study, synergism between the different members of the polymyxin B mixture following checkerboard analyses was explored. The tests revealed that the combination of polymyxins B3 and B1lle met the criteria for synergy against Enterobacteriaceae whereas the major components polymyxin B1 and B2 showed a low probability of synergy when combined. ${ }^{42}$

Regarding the pharmacokinetics of individual polymyxin B major components, no considerable differences were detected among them when tested in the rat animal model and in humans. In fact, it has been proposed as a reasonable approach to use the combined concentration values of the individual polymyxin components in pharmacokinetic studies to estimate overall drug exposure to polymyxin $\mathrm{B}^{43}$

In another study comparing polymyxin $B$ and colistin in the rat model, the individual major components of polymyxin B (B1 and B2) and colistin (A and $B$ ) yielded similar pharmacokinetic parameters such as clearance, volume of distribution, elimination half-life, and urinary recovery. Notwithstanding that, colistin A (polymyxin E1) and colistin $B$ (polymyxin E2) displayed lower protein binding in rat plasma compared to polymyxins B1 and B2.

In relation with the accumulation of the individual products (B1, B1Ile, B2+B3) in kidney, a fact that correlates with nephrotoxicity (see section 10 below), the relative proportions of the components present in the renal tissue at $48 \mathrm{~h}$ were found to be comparable to the concentrations in the USP (United States Pharmacopeia) mixture indicating no preferential accumulation of any of the components. ${ }^{44,45}$

Commercial polymyxin B is available in the sulfate form either for parenteral (intravenous and intramuscular), topical (ophthalmic and otic instillation), and intrathecal use (in cases of MDR Gramnegative caused meningitis). The dosage of intravenous polymyxin $B$ is generally $1.5-2.5 \mathrm{mg} / \mathrm{kg} /$ day $(15.000-25.000 \mathrm{IU} / \mathrm{kg} / \mathrm{day} ; 1 \mathrm{mg}$ of polymyxin B corresponds to ca $10.000 \mathrm{IU}$, International Unit), However, commercial formulations of polymyxin B are not always available in many countries of the world. Injectable polymyxin $B$ and colistin formulations are available only in Brazil, Malaysia, Singapore and the USA while in Europe and Australia, colistimethate is the only parenteral formulation that can be found. For the treatment of eye infections caused by $P$. aeruginosa, 0.1 $0.25 \%$ polymyxin $B$ solutions (10.000 IU to $25.000 \mathrm{IU} / \mathrm{ml}$ ) are recommended. Polymyxin $B$ in combination with a local anaesthetic (i. e. lidocaine, procaine) can also be found for intramuscular administration, in eardrops, and ointments. Combination with hydrocortisone is also available for otic use. ${ }^{15,46}$

Colistin has two commercially available forms: colistin sulfate and sodium colistimethate (sodium colistin methanesulfonate, CMS, Figure 2). ${ }^{47}$ Both contain different proportions of colistin $A$ (polymyxin E1), and colistin B (polymyxin E2), which account for more than $80 \%$ of colistin, together with many other minor components. ${ }^{6}$ Colistin sulfate may be administered orally for bowel decontamination or topically as a powder for the treatment of
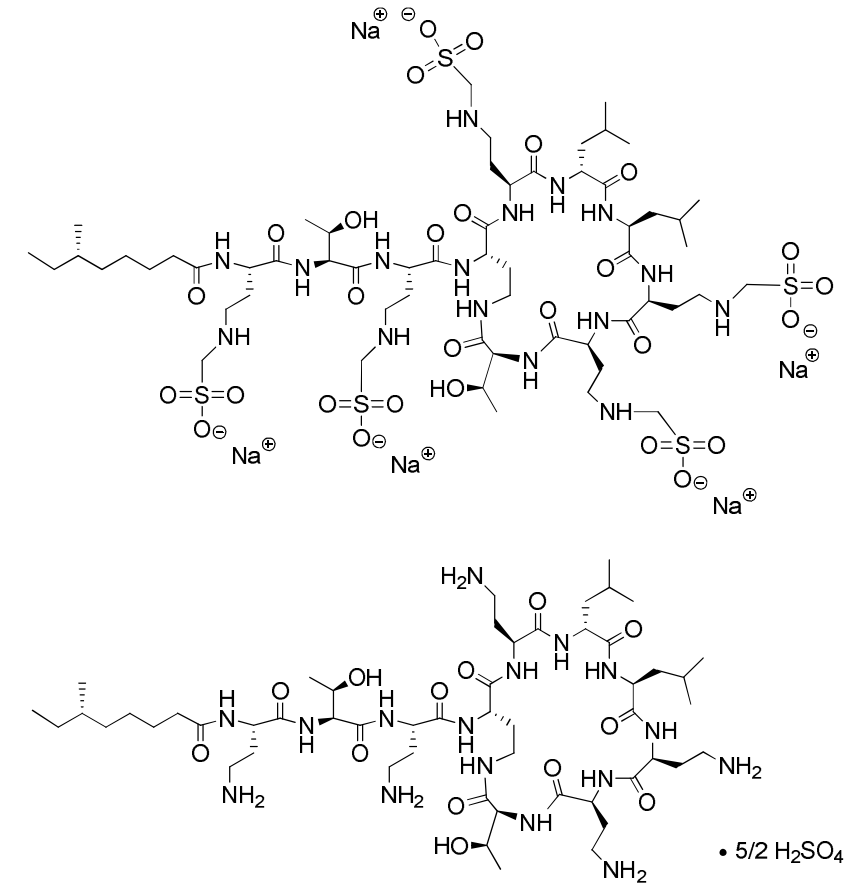

Figure 2: Structure of sodium colistimethate and colistin A (polymyxin E1). Molecular weight of colistimethate is 1749.81 g.mol ${ }^{-1}\left(\mathrm{C}_{58} \mathrm{H}_{105} \mathrm{~N}_{16} \mathrm{O}_{28} \mathrm{~S}_{5} \mathrm{Na}_{5}\right)$ while for the free base (devoid of sodium methylsulfonate derivatisation) it is $1169.48 \mathrm{~g}$ g. mol ${ }^{-1}$ $\left(\mathrm{C}_{53} \mathrm{H}_{100} \mathrm{~N}_{16} \mathrm{O}_{13}\right)$. Hence, $1 \mathrm{mg}$ of colistimethate corresponds to 0.67 $\mathrm{mg}$ of free base colistin (or $0.81 \mathrm{mg}$ of colistin sulfate, assuming 2.5 mols of sulfate per mol of colistin)

bacterial skin infections. Colistimethate is a prodrug of colistin. It is produced by the reaction of colistin with formaldehyde and sodium hydrogensulfite (see section 10 below). It is less toxic than colistin sulfate since it is polyanionic, but devoid of antimicrobial activity. It can be administered parenterally (intravenously, intramuscularly), intrathecally, intraventricularly or by inhalation (aerosolized, to treat respiratory tract infections caused by multidrug-resistant Gram-negative bacteria, and cystic fibrosis). Colistimethate as a prodrug, slowly reverts to colistin although hydrolysis is not always complete. ${ }^{48,49}$ It has been reported that only a $31.2 \%$ of $\mathrm{CMS}$ is hydrolysed in vitro to colistin in 4 hours at $37^{\circ} \mathrm{C}$ in human plasma. ${ }^{50}$ In vivo, even smaller proportions of colistimethate are converted to colistin. ${ }^{51}$ This has been attributed to the slow hydrolysis rate of colistimethate to colistin combined with a fastest rate of renal clearance. In fact, it is estimated that only ca $25 \%$ of colistimethate is converted to active colistin in patients with normal renal function. ${ }^{52}$

Colistimethate intravenous dosage in adult patients with normal renal function are different in Europe and USA. In Europe colistimethate doses are in general 4-6mg/kg (50.000-75.000 IU/kg daily; $1 \mathrm{mg}$ of colistimethate corresponds to $12.500 \mathrm{IU})$. In France and Austria, it reaches values of $12 \mathrm{mg} / \mathrm{kg}$ (150000 IU/ $/ \mathrm{kg} /$ day). In the USA, the recommended dose is $2.5-5 \mathrm{mg} / \mathrm{kg}$ expressed in terms of colistin base, equivalent to $6-12 \mathrm{mg} / \mathrm{kg}$ of colistimethate, $(75000$ $150000 \mathrm{IU} / \mathrm{kg})^{46,54}$ 
Examples of polymyxin drug brands approved by the FDA and present in the market include Pediotic ${ }^{\circledR}$ (neomycin, polymyxin B sulfate and hydrocortisone suspension, USP, for otic use), Polysporin ${ }^{\circledast}$ (polymyxin B, zinc bacitracin and gramicidin; aerosol, topical, and ophthalmic), Neosporin ${ }^{\circledast}$ (polymyxin B, zinc bacitracin and neomycin triple ointment) and Polytrim ${ }^{\circledR}$ (polymyxin B sulfate and trimethoprim ophthalmic solution, USP). Colomycin ${ }^{\circledR}$ and Coly-Mycin $^{\circledast}$ contain colistin methanesulfonate as the active principle (1-2 million units, for injection). Polymycin B sulfate (polymyxin B sulfate) was approved by the FDA in 2011 for the treatment of infections caused by resistant strains of $P$. aeruginosa, $H$. influenzae, E. coli, Enterobacter aerogenes, and $K$. pneumoniae by parenteral administration. ${ }^{32}$

\section{Mechanism of action}

Polymyxins have a narrow antimicrobial spectrum with selectivity for Gram-negative bacteria. This is because the first molecular target of these polycationic lipopeptides is the lipopolysaccharide (LPS), the main component of the outer membrane (OM) of Gramnegatives. The low permeability of the two-membrane cell envelope in Gram-negative bacteria is the main reason for the low hit rate in the discovery of new antibiotics against this bacterial class. ${ }^{55}$ The outer membrane is an asymmetric bilayer of LPS in the outer monolayer, and a mixture of phospholipids in the inner. ${ }^{56,57}$ LPS is composed of three domains: lipid A, central core oligosaccharide, and outermost $\mathrm{O}$-antigen chain. ${ }^{58}$ Lipid $\mathrm{A}$ is the domain responsible for outer membrane thigh packing, and the principal target for polymyxins. It contains an $\mathrm{N}$ - and O-acylated diglucosamine bisphosphate backbone, and forms a highly packed structure. Although there are many variations among species, in polymyxin-sensitive bacteria LPS has several anionic charges, responsible for the strong electrostatic interactions with polycationic polymyxins. LPS molecules are bridged and partly neutralized by divalent ions $\mathrm{Ca}^{2+}$ and $\mathrm{Mg}^{2+}$, thus conferring a high rigidity and low permeability to the outer membrane. ${ }^{56,59}$

The antimicrobial activity of polymyxins begins by competitive displacement of membrane-stabilizing divalent cations $\mathrm{Ca}^{2+}$ and $\mathrm{Mg}^{2+}$, thus causing a destabilization of the LPS layer and allowing insertion of the hydrophobic acyl chain of the antibiotic, which locates in the hydrophobic domain of lipid $A .{ }^{60,61}$ This causes an expansion of the LPS monolayer ${ }^{15,62}$ and results in disruption of the outer membrane permeability barrier, facilitating the entrance of polymyxin into the periplasmic space, a process of self-promoted uptake first described by Hancock. ${ }^{63,64}$ Binding to LPS is a required first step for antibacterial activity, but it is not enough. For example, deacylated polymyxin $\mathrm{B}$ nonapeptide, lacking the $\mathrm{N}$-terminal acyl chain and $\mathrm{Dab}^{1}$ residue, is an extremely poor antibiotic, but is still capable of binding to LPS and preserving a significant OMpermeabilizing action. ${ }^{14}$ This susceptibility explains the drastic sensitizing action of the nonapeptide, allowing other small molecules (such as conventional antibiotics) to cross the outer membrane. ${ }^{65}$ In addition, polymyxin resistance is related to lipid A modification with phosphoethanolamine and/or galactosamine, or to the complete loss of LPS, thus avoiding binding of polymyxins to the $\mathrm{OM}^{66}$
The interaction of PxB and PxB nonapeptide with LPS has been studied in detail at the molecular level, and involves hydrophobic as well as electrostatic interactions. The structure of PxB bound to LPS has been determined by NMR spectroscopy, ${ }^{67-70}$ and consists in an envelope-like fold of the peptide ring separating the polar/charged residues from the hydrophobic components, conferring an amphiphilic character to the structure. It is postulated that the $\beta$ turn structure is stabilized by hydrophobic interactions involving two hydrophobic domains on the lipopeptide DPhe ${ }^{6}-$ L-Leu $^{7}$ domain on the peptide ring, and the $\mathrm{N}$-terminal fatty acid chain, with the aliphatic chains of lipid $A .^{65,68}$ The electrostatic interactions between the positive side chains of Dab residues on PxB and two of the negative phosphate groups of the phosphorylated lipid $A$ headgroups are essential for complex formation, whereas the hydrophobic interactions are responsible for insertion into the outer membrane hydrophobic core. The structure of PxB nonapeptide bound to LPS has been determined by tranferred nuclear Overhauser effect NMR and molecular dynamics, ${ }^{70}$ and is consistent with surface binding of the peptide, without insertion into the hydrophobic core of lipid A. This will explain the lack of antibiotic activity, since the nonapeptide will not reach the inner membrane.

Once polymyxin has crossed the outer membrane, it must interact with the cytoplasmic or inner membrane in order to kill the bacteria. ${ }^{11}$ The inner membrane in Gram-negative bacteria is mostly composed of zwitterionic phospholipid phosphatidylethanolamine (PE) and anionic phosphatidylglycerol (PG) and cardiolipin (CL). All bacteria have at least a $15 \%$ of anionic lipids, but this can be either PG or $\mathrm{CL}$ or both. ${ }^{57}$ The same lipids are found in the inner layer of the outer membrane, although the proportions are different. ${ }^{71}$ The mechanism of bacterial killing is not related with membrane permeation, which takes place at concentrations well above the minimal inhibitory concentration. ${ }^{62,72,73}$ A threshold concentration of $\mathrm{PxB}$ is required on the membrane to form clusters that insert and form depolarizing ion-permeable pores, however dissipation of the $\mathrm{pH}$ gradient is not observed in E. coli after PxB treatment, and the bactericidal effect is expressed at lower concentrations and is not dependent on depolarization of the outer membrane. ${ }^{74}$ Although a mechanism of bacterial killing based on disruption of the physical integrity of the cytoplasmic membrane by pore formation or even a detergent effect are demonstrated for other $\mathrm{AMPs}^{75}$ in the polymyxin family such effects only occur at high peptide/lipid ratios.

A more likely mechanism of action has been described for polymyxin B that involves contact formation between the outer and inner membranes of Gram-negative bacteria, ${ }^{71,76}$ also seen in other antimicrobial peptides such as cecropins, ${ }^{77}$ and $\mathrm{rBPI}_{21}{ }^{78}$ According to this model, once in the periplasmic space stoichiometric amounts of polymyxin will form contacts between the two enclosed phospholipid interfaces, and promote a fast and selective exchange of anionic phospholipids. The resulting changes in the membrane lipid composition trigger an osmotic imbalance that leads to bacterial stasis and cell death. ${ }^{79}$ Biophysical studies using model membranes have demonstrated that at the concentrations around the MIC, PxB and colistin induce the apposition of anionic vesicles with a composition that mimics the bacterial membrane, and the 
formation of functional vesicle-vesicle contacts. ${ }^{80}$ These contacts support a fast and selective exchange of phospholipids exclusively between the outer monolayers of the vesicles in contact and maintaining intact the inner monolayers and the aqueous contents. ${ }^{81}$ For example, monoanionic phospholipids such as phosphatidylglicerol are transferred through the contacts, whereas zwitterionic phosphatidylcholine or dianionic phosphatidic acid are excluded, independently of the composition of the fatty acid chains. The non-antibiotic derivative polymyxin B nonapeptide is not able to induce vesicle-vesicle contacts. ${ }^{82}$ Sublethal concentrations of $\mathrm{PxB}$ in growing E. coli induce a highly selective cellular stress, with transcription of the osmY gene without leakage of solutes and protons. ${ }^{71,77,79}$ Since osm $Y$ expression is also induced by hyperosmotic stress, encoding a periplasmic protein that protects from cell membrane damage, ${ }^{83}$ the interpretation is that PxB forms functional contacts in the periplasmic space between the anionic phospholipid-containing outer surface of the cytoplasmic membrane and the inner surface of the outer membrane. ${ }^{84}$ The consequent loss of phospholipid compositional specificity caused by the PxB-mediated exchange can be the origin of the osmotic imbalance that leads to bacteriostasis and cell death. ${ }^{14}$ An analysis of the transcriptome of $A$. baumannii exposed to colistin shows that this antibiotic alters the expression of a very large number of genes, many of them involved in the synthesis and transport of membrane components. This is consistent with the inner membrane-outer membrane lipid exchange mechanism of action with alteration of the normal membrane composition. ${ }^{85}$

The structure of polymyxin in the IM-OM contacts has been characterized in lipid vesicles by fluorescence resonance energy transfer, using labelled derivatives of a synthetic PxB analog, sp-B. ${ }^{86}$ Intermolecular FRET is consistent with self-association of the peptide, possibly forming dimers, when bound to anionic vesicles at concentrations that are relevant for formation of vesicle-vesicle contacts and lipid exchange. Atomic force microscopy of polymyxin bound to monolayers of $E$. coli lipid extract shows structures that are consistent with the formation of aggregates of several particles at the concentrations that induce contact formation. ${ }^{82}$

A series of polymyxin analogs obtained by solid phase synthesis and including a disulfide bond gave additional information on the mechanism of action. For example, conservative analogs maintaining the main structural characteristics of polymyxin, namely 5 positive charged residues, a cyclic heptapeptide, a lineal tripeptide and the $\mathrm{N}$-terminal acyl chain, are also active against Gram-negative bacteria and induce vesicle-vesicle contacts and a selective lipid exchange similar to polymyxin. ${ }^{87,88}$ However, substitution of Dab residues for Arg results in more lytic lipopeptides, with a different spectrum of activity that includes Gram-positive and Gram-negative bacteria. ${ }^{89,90}$ Flow citometry of $E$. coli treated with one of the Arg-containig polymyxin analogs shows that depolarization and permeabilization take place roughly at the same time, and are consistent with a membrane-based mechanism of action. ${ }^{89,91}$ It has also been shown that polymyxin B and colistin are able to inhibit the vital respiratory enzyme type II NADHquinone oxidoreductase (NDH-2) in the inner membrane of Gramnegative bacteria, although $\mathrm{IC}_{50}$ values are high. ${ }^{92}$

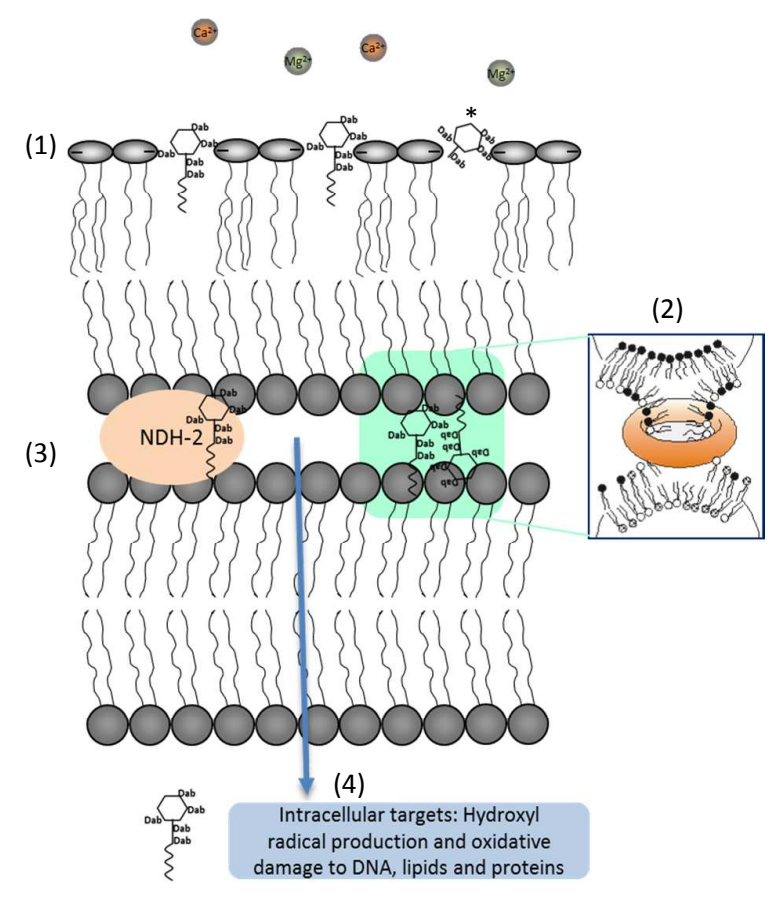

Figure 3: Representation of the putative mechanism of action of polymyxin on Gram-negative bacteria. (1) Displacement of $\mathrm{Ca}^{2+}$ and $\mathrm{Mg}^{2+}$ and binding to lipid A; (2) Self-promoted uptake to the periplasmic space and formation of OM-IM contacts and lipid exchange; (3) Inhibition of respiratory enzyme type II NADH-quinone oxidoreductase; (4) Entry into the cytoplasm and access intracellular targets. *Polymyxin B nonapeptide activity is limited to step (1).

Interestingly, polymyxin B nonapeptide has no inhibitory activity, in agreement with its reported inability to cross the cell membrane. $\mathrm{NADH}-2$ inhibition has also been identified in other compounds including phenothiazines, quinolinyl pyrimidines and quinolones, but in all cases the mode of action remain unclear and in the case of polymyxins is considered a secondary mechanism of action. ${ }^{93}$ Polymyxin $B$ and $E$ can inhibit alternative membrane bound respiratory enzymes nicotinamide adenine dinucleotide dehydrogenase and malate:quinone oxidoreductase in Mycobacterioum smegmatis. ${ }^{36}$

Some reports suggest that entry into the cytoplasm of the bacterial cell is not necessary for activity. For example, polymyxin B covalently attached to agarose beads has a good antimicrobial activity on $E$. coli and $P$. aeruginosa. ${ }^{94}$ It is proposed that perturbation of outer membrane structure by polymyxin-agarose indirectly affected the selective permeability of the inner membrane and inhibited respiration. However, the chemistry followed in this study did not provide selectivity to the anchoring point on the peptide, given that it includes multiple free amines, any of which could have reacted with the spacer arm bound to the agarose beads. A better example is a cysteinilated derivative of 
battacin, a close analog of polymyxin that retains its activity when covalently linked to a derivatized solid surface, being a promising agent as antibacterial surface coatings to prevent bacterial colonization and biofilm formation. ${ }^{95}$ The lipopeptide-coated surfaces caused significant damage to the cellular envelope of $P$. aeruginosa and $E$. coli upon contact and prevented surface biofilm colonization.

It should be stressed that the mechanism of action of polymyxins on Gram-negative bacteria based on $\mathrm{OM}$ and IM interactions is well documented. However, other mechanisms involving intracellular targets can also play a role. ${ }^{15,96}$ Recently, entry of polymyxin into the cytoplasm of Gram-negative bacterial cells has been demonstrated by time-lapse laser scanning confocal microscopy using a dansylated polymyxin $B$ that maintains the pharmacological properties of the natural antibiotic. ${ }^{97}$ In their work, the authors show that labelled polymyxin initially accumulated in the OM of $K$. pneumoniae, then it gradually penetrated the OM and accessed the IM, and only at high concentrations $(5 \times \mathrm{MIC})$ it became homogeneously distributed in the cytoplasm. The possibility of intracellular targets for polymyxins is not clear, but there are some studies that point in that direction. For example, the generation of hydroxyl radical production by the Fenton reaction leading to the formation of hydroxyl radicals through the reduction of hydrogen peroxide by ferrous ion $\left(\mathrm{Fe}^{2+}\right)$ has been observed in several Gramnegative species, including $A$. baumanii and $E$. coli, ${ }^{76,98}$ and $K$. pneumoniae. ${ }^{99}$ The production of reactive oxygen species (ROS) is concurrent with the rapid killing of these bacteria by both polymyxin $B$ and colistin, probably by oxidative damage in the bacterial DNA, proteins and lipid. ${ }^{100}$ In support of this intracellular mechanism of action, a study by Pournaras et al. ${ }^{101}$ shows that in a colistin-resistant isolate of $A$. baumanii there is a significant decrease in the expression of enzymes involved in oxidative stress response. An increase in expression of genes encoding superoxide dismutase enzymes after colistin treatment in $A$. baumanii also agrees with the idea of hydroxyl radicals being involved in colistin antibacterial activity. ${ }^{85}$

\section{Resistance to polymyxins}

The therapeutic rescue of polymyxins for their use in nosocomial infections has been followed by an emergence of acquired resistance among the most clinically relevant Gram-negative bacteria. Resistance to polymyxin is a complex subject that would require of another thorough review by itself. Hence, only a brief mention will be made here. Several recent reviews summarizing the mechanisms of resistance to polymyxins are also available. ${ }^{53,102-105}$ As seen before, the first molecular target of polymyxins in the bacterial surface is the LPS of the outer membrane. Since electrostatic interactions are established with anionic phosphate groups in lipid A, modification of those with positively charged groups such as phosphoetanolamine or 4-amino-4-deoxyL-arabinose provides a mechanism of protection from the interaction with polycationic polymyxin and thus, of resistance. ${ }^{106}$ Changes in lipid A may include deacylation, hydroxylation and palmitoylation. Other mechanisms of resistance include the utilization of efflux pumps and capsule formation. Resistance is mainly adaptive (reversible) and regulated by two-component systems (e. g. PhoP/PhoQ and PmrA/PmrB) and can be triggered by environmental stimuli (low $\mathrm{Mg}^{2+}$ levels, sublethal concentrations of AMPs, for instance). Recently, resistance to colistin due to plasmidmediated mcr-1 gene has also been described. MCR-1 is a phosphoethanolamine transferase enzyme (it adds phosphoethanolamine to lipid A). Resistance to polymyxins is certainly an added challenge to the development of new antibiotics against pan-drug resistant Gram-negative bacteria. ${ }^{107}$

\section{Combination with other antibiotics}

The main interest of polymyxin combinations with other antibiotics lies in the treatment of infections caused by resistant and multidrug-resistant bacteria whose proliferation is becoming a serious social and economic problem worldwide and account for growing global morbidity and mortality. Multidrug-resistant pathogens are considered those that are resistant to three or more antibiotic classes. The worst are the extensively drug-resistant (XDR) ones, particularly those Gram-negative pathogens that are non-susceptible to all but one or two antibiotic classes. ${ }^{108}$ In this situation, when even carbapenems, a major last-line class of antibiotics to treat bacterial infections, are not useful polymyxins have been rescued and become last-resort agents against XDR $P$. aeruginosa, K. pneumoniae and A. baumannii.

The emergence of polymyxin-resistant strains and polymyxin heteroresistance (heterogeneity of response to antibiotics from bacterial cells within the same population) is questioning the utility of polymyxin monotherapies. Increasing the dose to maximize efficacy of the treatment is not viable since polymyxins are nephrotoxic and exhibit a narrow therapeutic index. An alternative option would be the administration of polymyxins in combination with other antibiotic agents or non-antibiotic compounds. ${ }^{109}$ The mechanism of action of polymyxins, that affects the integrity and enhance permeability of the outer membrane of Gram-negative bacteria, may help increase activity and accumulation ${ }^{110}$ within bacterial cell of other antibiotic classes.

Several studies have explored the synergistic activity of polymyxins with other antimicrobial agents against Gram-negative bacteria, particularly $P$. aeruginosa, $K$. pneumoniae and $A$. baumannii. Synergy may be assessed in vitro by three methods: time-kill studies, Etest and microdilution. The major interest of combinations is to show synergistic activity against resistant bacterial strains to at least one of the antibiotics, chiefly the one showing the highest MIC. One of the antibiotic classes most commonly used in combination with polymyxins is the carbapenem family. In a systematic review and meta-analysis, combination therapy following the time-kill method showed synergy rates of $44 \%$ (30 to $59 \%$ ) for K. pneumoniae, 50\% (30 to 69\%) for P. aeruginosa and $77 \%$ (64 to 87\%) for $A$. baumannii. Of the carbapenems studied, doripenem showed high synergy rates for all three bacteria. Meropenem was more synergistic for $A$. baumannii and imipenem for $P$. aeruginosa. Etest and checkerboard assays generally yielded lower synergy rates than time-kill studies. ${ }^{111}$ 
In addition to carbapenems, combinations of polymyxins with amikacin, ${ }^{112}$ ceftazidime, ${ }^{113,114}$ ciprofloxacin, ${ }^{114}$ fosfomycin, ${ }^{115-117}$ gramicidin $^{118,119}$ or rifampicin ${ }^{120}$ have been described to confer additive bactericidal activity against several $P$. aeruginosa strains in vitro. Regarding $K$. pneumoniae, efforts have been mostly devoted to $K$. pneumoniae carbapenemase (KPC) and metallo- $\beta$-lactamase (MBL) producing strains. ${ }^{121}$ Synergistic activity has been observed in combinations with fosfomycin, ${ }^{115,122,123}$ vancomycin, ${ }^{124}$ rifampicin, ${ }^{125,126}$ tygecicline, ${ }^{126}$ chloramphenicol, ${ }^{127}$ plazomycin, ${ }^{128}$ and aztreonam. ${ }^{129}$

The combination therapy of polymixin with rifampicin is one of the most tested options for the treatment of MDR and XDR Gramnegative bacterial infections, and $A$. baumannii is no exception. ${ }^{130-134} \mathrm{~A}$ lot of attention has also received the synergistic studies of polymyxins with glycopeptides (teicoplanin, vancomycin, telavancin). ${ }^{124,135-141}$ Partial synergy has been observed with azithromycin. ${ }^{142}$ Synergistic effectiveness of colistin with meropenem and sulbactam, ${ }^{143}$ minocycline ${ }^{144}$ and daptomycin, an anionic lipopeptide, has also been shown. ${ }^{145}$ Finally, the combination of lantibiotic nisin (normally used as a food preservative) with either colistin or polymyxin B yielded a pronounced synergistic effect in E. coli, K. pneumoniae, P. putida and $P$. aeruginosa. ${ }^{146}$ However, no additive effect was found with nisin in binary combination with penicillin, erythromycin or chloramphenicol.

In summary, numerous studies have identified various polymyxin combinations presenting synergistic activity against sensitive and multi-drug resistant $P$. aeruginosa, $K$. pneumoniae and $A$. baumannii. Apparently, synergy was more evident in polymyxinresistant strains, what augurs well for the combination therapy in front of polymyxin monotherapy. In addition, regrowth and emergence of resistance is consistently seen in polymyxin monotherapy. Given this situation, polymyxin combination therapy could reduce the possibility for selection of resistant subpopulations or the development of new resistance. Although most in vitro data endorse this view, clinical investigations of polymyxin combination therapy are in its infancy. So far, clinical data is apparently inconclusive in showing evident superiority of the cotherapy due to the small sample size studies, among other reasons. ${ }^{53,147-149}$ To overcome this issue, well-designed clinical tests are urgently needed to give a clear answer. In this regard, two large clinical trials comparing colistin monotherapy and colistin combined with meropenem are currently in progress both in Europe and the US. $^{150}$

\section{Synergy with antifungals}

Polymyxins are known to have a poor fungicidal activity (MIC $\geq 8$ $\mathrm{mg} / \mathrm{L}$ ). However, the synergistic antifungal properties of polymyxin B were studied as early as 1972 . Polymyxin was found to potentiate the activity of tetracycline in Candida albicans and Saccharomyces cerevisiae, even at low concentrations. Polymyxin B seemed to increase the permeability of the yeast cell membrane to tetracycline, which then inhibited protein synthesis and led to cell death. ${ }^{151}$ More recently, it has been demonstrated that the polymyxin B combination with fluconazole or itraconazole was active at low concentrations against Aspergillus fumigatus, Rhizopus oryzae, Candida albicans and non-albicans Candida species. The combination at clinically relevant low concentrations was particularly potent against Cryptococcus neoformans, including strains resistant to fluconazole. ${ }^{152}$ Polymyxin $B$ has also been demonstrated to reduce the tissue fungal burden both in intravenous and inhalation models of murine cryptococcosis at a level comparable with that of fluconazole. ${ }^{38}$

Synergistic antifungal activity against $C$. albicans has also been reported when polymyxin $B$ was combined with amphotericin $B$, ketoconazole and miconazole. ${ }^{153,154}$ Similarly, colistin has also been found to act synergistically with amphotericin B against $R$. oryzae. ${ }^{155}$ Colistin, both as a single agent or in combination with voriconazole, caspofungin and amphotericin $\mathrm{B}$, has also shown in vitro antifungal activity against filamentous ascomycetes occurring in cystic fibrosis patients and may offer new therapeutic options, especially for multidrug-resistant Scedosporium prolificans. ${ }^{156}$

In a recent study to assess the in vitro susceptibility of 25 clinical isolates of Fusarium to antifungal agents (amphotericin B, caspofungin, itraconazole and voriconazole) and antimicrobials (pentamidine, polymyxin B, tigecycline and tobramycin), the highest rates of synergism were observed when amphotericin $B$ or voriconazole were combined with tobramycin $(80 \%$ and $76 \%$, respectively), polymyxin B (76\% and $64 \%$ ) and pentamidine (72 \% and $68 \%) .{ }^{157,158}$

Finally, caspofungin and echinocandin antifungals in combination with colistin have also been found to act synergistically against fluconazole-resistant and susceptible C. albicans and C. glabrata isolates. However, authors also state that the correlation with in vivo benefits may not be straightforward. ${ }^{159-161}$

\section{Antibiofilm activity}

A biofilm is an organized microbial ecosystem that consist of one or more microbial species imbedded in a self-produced matrix of extracellular polymeric substances composed by proteins, polysaccharides and DNA. Biofilms can develop on human body tissues and surfaces of medical devices. Antibiotic treatments against biofilms usually require of high doses administered for long periods of time. Since current available antibiotics have been developed to target planktonic bacteria, they often fail to fight persistent infections associated with biofilms. ${ }^{162}$

Polymyxins have been proven to be active against biofilms, both as a single agent or in combination with other antibiotics, particularly against $A$. baumannii and $P$. aeruginosa. ${ }^{163,164}$ However, neither colistin nor polymyxin B were found capable of preventing (p)ppGpp accumulation (alarmones guanosine 5'-diphosphate 3'-diphosphate, ppGpp, and guanosine 5'-triphosphate $3^{\prime}$-diphosphate, pppGpp) signaling nucleotides that regulate the stringent response in bacteria and are known to play a role in biofilm formation. ${ }^{165}$ 
Polymyxin was found to show an antibiofilm synergistic interaction with cyclic antimicrobial peptide gramicidin $\mathrm{S}$ toward 17 multidrugresistant $P$. aeruginosa and biofilms of $P$. aeruginosa strain PAO1. The concentration of polymyxin $B$ required to inhibit biofilm formation by $P$. aeruginosa PAO1 was $8 \mu \mathrm{g} / \mathrm{ml}$. Treatment in combination with gramicidin S required only $2 \mu \mathrm{g} / \mathrm{ml}$ (gramicidin S concentration got reduced from $32 \mu \mathrm{g} / \mathrm{ml}$ to $4 \mu \mathrm{g} / \mathrm{ml}$ ). The FIC (fractional inhibitory concentration) calculated from this decrease was 0.375 , which indicated a synergistic effect of this treatment. ${ }^{118}$ Inhibition of biofilm formation by $P$. aeruginosa (PA-01 strain) has also been found between nisin lantibiotic and either colistin or polymyxin B. As a control, none of these antimicrobials inhibited biofilm formation when used individually. ${ }^{146}$

Colistin and tobramycin, both alone or in combination exhibited bactericidal activity prior to biofilm attachment to endotracheal tubes, however no activity was observed once biofilm formed on such polyvinylchloride tubes. ${ }^{166}$ Polymyxin B proved to be $100 \%$ effective in vitro against a highly prevalent clone of multi-drug resistant $A$. baumannii, with a $92.9 \%$ of strains being biofilm producers (associated patient lethality of $28.2 \%$ in a Mexican Tertiary Care Hospital). However, no direct proof of polymyxin inhibiting biofilm formation in this clone was provided. ${ }^{167}$

Recently, colistin entrapped in nanoparticles of different materials [poly (lactide-co-glycolide, chitosan, poly(vinyl alcohol] have been proven to eradicate pre-formed $P$. aeruginosa biofilms. Nanoparticles of colistin/poly(vinyl alcohol) and colistin/chitosan could penetrate inside the biofilms, release colistin in situ, thus increasing the effectiveness of the treatments. ${ }^{168,169}$ A synergistic or additive effect between colistin and levofloxacin has been reported in vitro and in Galleria mellonella model against colsitin-susceptible A. baumannii strains, although not against colistin-resistant ones. ${ }^{170}$

\section{Toxicity}

Toxicity of polymyxins has been reported since the beginning of its use. Toxicity is dose dependent and reversible once the treatment is discontinued. However, the exact molecular mechanism of toxicity is not well-understood.

In 1947, Stansly and coworkers already described the sulfomethylation of polymyxin to reduce acute toxicity, according to previous reports describing the significance of transforming a cationic drug into an anionic one:

$$
\mathrm{R}-\mathrm{NH}_{2}+\mathrm{HCHO}+\mathrm{NaHSO}_{3} \rightarrow \mathrm{R}-\mathrm{NH}-\mathrm{CH}_{2}-\mathrm{SO}_{3}{ }^{-} \mathrm{Na}^{+}+\mathrm{H}_{2} \mathrm{O}
$$

They also observed that this sulfomethylated form of polymyxin was free of producing painful irritation when administered subcutaneously or intramuscularly. ${ }^{47}$

The main adverse side effects of polymyxins are nephro- and neurotoxicity. ${ }^{171,172}$ The rate of colistin-associated neurological toxicity is approximately $7 \%$. The major manifestation of neurotoxic side effects after parenteral colistimethate administration is paresthesia ("pin and needles sensation"). Neuromuscular blockade can also occur during polymyxin therapy although no episodes have been reported in the literature in the last years. Ataxia, vertigo, confusion, dizziness, weakness, visual, speech disturbances, hallucinations and seizures are also possible during polymyxin therapy.

The major toxicological problem of polymyxins is nephrotoxicity. Both polymyxin B and colistin/colistimethate are known to produce adverse side effects in kidney. ${ }^{171,172}$ Nephrotoxicity rates typically range between $10 \%$ and $60 \%$. In the largest clinical study so far performed (258 patients) the rate of nephrotoxicity was found to be $10 \%{ }^{173}$ In a series of recent studies carried out in cohorts of 71 and 66 patients, acute kidney injury (AKI) rates associated to colistimethate administration were found to be $45-56 \%$ according to the Risk, Injury, Failure, Loss and End-stage (RIFLE) kidney disease criteria. ${ }^{174,175}$ In a study performed in Korea, AKI incidence was found to reach $54.6 \%$ in a cohort of 119 patients. ${ }^{176}$ In another study involving 92 patients comparing a high-dose of colistimethate treatment $(9 \mathrm{MIU}$, million IU, followed by a maintenance dose of 4.5 MIU/12 h) versus the standard dose (6 MIU/day), a higher cure rate in the high-dose group was found ( 63 vs. $41.3 \%, p=0.04$ ) with no higher risk of nephrotoxicity (32.2 versus $26 \%) .{ }^{177}$ In a smaller study involving 30 patients in Orlando (Florida, US), nephrotoxicity occurred in 3 out of $10(33 \%)$ of patients. An interesting point of this study was that excessive colistimethate dosing was frequent $(47 \%)$, often due to the higher body weight in obese patients $(71 \%)$, and was associated with higher rates of nephrotoxicity ( $80 \%$ versus $30 \%)$. Overall, a close monitoring of the renal function in patients is mandatory to identify the optimal colistimethate dose from both efficacy and toxicity perspectives. ${ }^{178}$ The use of homogeneous criteria, such as the RIFLE one, when carrying out nephrotoxicity studies was also found to be necessary for the evaluation of acute kidney injury and allow for comparison of nephrotoxicity among reports.

The use of potentially nephroprotective agents has been advocated to ameliorate adverse side effects of polymyxins (ascorbic acid, vitamin $\mathrm{E}$, melatonin, lycopene, $\mathrm{N}$-acetylcysteine). In rodent in vivo models, some positive results showed that coadministration of antioxidants can protect against colistin-induced nephrotoxicity. ${ }^{179}$ In the clinic, administration of intravenous ascorbic acid has been studied as a potentially useful component to prevent nephrotoxicity because of its antioxidant properties. However, evidence of a protective effect is not conclusive according to two clinical studies. $^{180,181}$

Since both polymyxins and colistimethate are nephrotoxic, a question rises regarding the relative toxicity of the free base in comparison with the methansulfonated derivative. Again, results are not conclusive. In a report by Oliveira in Brazil dealing with the treatment of infections (41 patients) caused by carbapenemresistant Acinetobacter spp, both polymyxins gave equivalent results with regard to efficacy and toxicity. ${ }^{177}$ In a systematic review and meta-analysis, unadjusted nephrotoxicity was more common in patients treated with colistin than with polymyin B, but according to the RIFLE criteria, there was no difference regarding risk, injury or failure between colistin and polymyxin B. ${ }^{183}$ Two other studies though, reported that nephrotoxicity rates were lower with 
polymyxin B than colistimethate. ${ }^{171,184,185}$ Also, polymyxin B seems to have some advantage over colistimethate regarding rapid target concentration attainment and antibacterial activity. ${ }^{186}$

\subsection{Mechanism of nephrotoxicity}

The mechanism of polymyxin-induced nephrotoxicity is not clear and numerous efforts are carried out to uncover it. ${ }^{187,188}$ The structure-activity data on polymyxin analogs seem to indicate that toxicity is related to the amphipathic nature of the molecule, due to the presence of hydrophobic residues (fatty acid, amino acids 6 and 7) and particularly due to the presence of charged Dab side chains at physiological $\mathrm{pH}$. Polymyxins accumulate within proximal tubular epithelial cells (PTEC) of the kidney via endocytosis at the multiligand receptor megalin. Megalin is highly expressed on the luminal surface of renal proximal tubules. It is a negatively charged protein involved in the binding and endocytosis of polybasic molecules such as aprotinin, cytochrome cor aminoglycosides. ${ }^{189,190}$ The mechanism of nephrotoxicity of polymyxins has been proposed to be similar to that described for aminoglycosides. ${ }^{191,192}$

Polymyxin-induced nephrotoxicity has been associated with acute tubular necrosis in kidneys and rise of creatinine levels in blood. Serum creatinine increments after parenteral administration of colistimethate are dose- and duration-dependent as proven in rats and humans. Phamacokinetic data indicate that polymyxins are extensively reabsorbed by active transport processes in the renal tubules after filtration at the glomerulus (from tubular urine back into blood). ${ }^{193}$ This major tubular reabsorption may induce an accumulation of the drug in the tubular cells and this could be, at least in part, a potential cause for nephrotoxicity. Accumulation of polymyxin induces apoptosis in kidney tubular cells ${ }^{194}$ and may be the result of mitochondrial damage and/or release of reactive oxygen species. ${ }^{187}$ The urinary recovery of polymyxin B and colistin is less than $1 \%$, as proven in humans and rats, respectively. Thus, it is evident that nonrenal elimination is the predominant clearance pathway for both polymyxins. However, the urinary recovery of sodium colistimethate can be higher than $60 \%$ (as seen in assays in rats, dogs and humans). The explanation may come from the different ionic nature of both polymyxins (polycations) and colistimethate (a polyanion). Urinary concentrations of colistin after administration of colistimethate can be relatively high as a result of hydrolysis within the urinary tract from colistimethate that is extensively renally excreted (and not reabsorbed). Partially sulphomethylated derivatives of colistin are also excreted before the full hydrolysis and are not reabsorbed. ${ }^{195-197}$

Recently, a detailed study by Mass Spectrometry Imaging (MSI) helped to reveal the distribution of polymyxin B1, colistin, and the less nephrotoxic truncated derivative, polymyxin $B$ nonapeptide within rat kidney. ${ }^{198}$ The bioanalysis showed that polymyxin B1 and colistin preferentially accumulated in the renal cortical region while polymyxin B nonapeptide, a less nephrotoxic compound, was more uniformily distributed throughout the kidney. Ratios of relative proportions of kidney cortex:medulla at seven days were 5.8 to 1

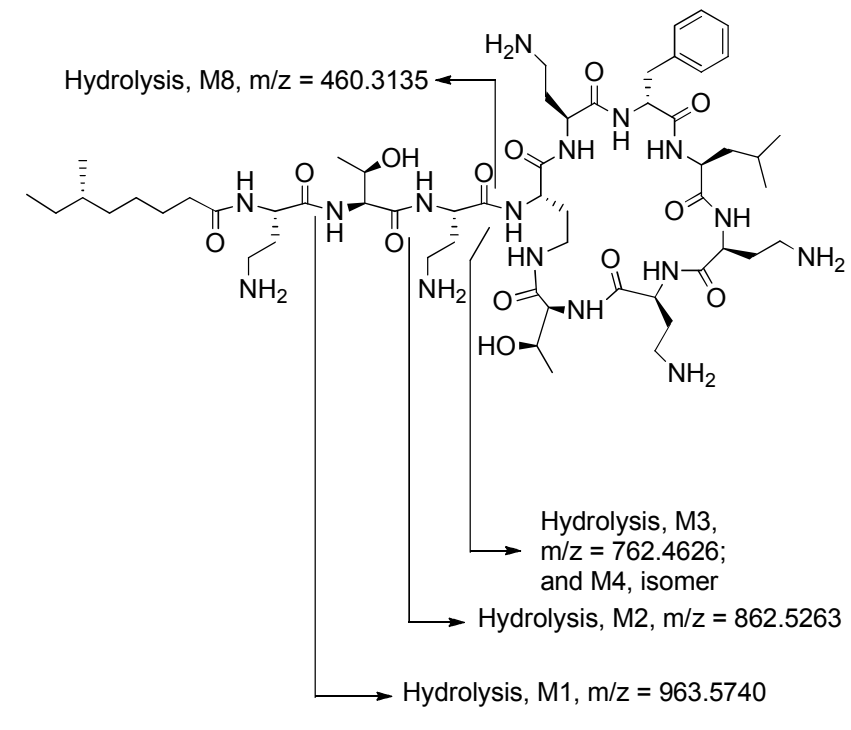

Minor metabolization: demethylated, oxydized polymixin metabolites M6, M7, M9, and M10 m/z = 1203,7213

Figure 4: Polymyxin B1 metabolites found in renal cortex in addition to polymyxin B1 itself. Metabolites were identified by LC/MS/MS (Liquid Chromatography, Mass Spectrometry) according to Nilsson and coworkers (M5, unknown structure). Arrows indicate peptide bonds metabolized by hydrolysis. ${ }^{198}$

for colistin and 25.4 to 1 for polymyxin B. For polymyxin nonapeptide, the ratio was 2.9 to 1 . These results correlate with recent immunohistochemical and correlative microscopy studies, which also demonstrated that polymyxins accumulate within renal tubular cells of the renal cortex. ${ }^{199-202}$ In addition, metabolites that accumulate with a similar distribution as the parent polimixins have also been identified by high resolution MS (Figure 4).

Both tissue homogenates and urine samples were analyzed. Ten polymyxin metabolites were identified within the tissue homogenates, six of which were also found in the urine sample. No quantification of the relative abundances of the different metabolites was provided, though. Nevertheless, it is interesting to see that both non-metabolized polymyxin and colistin accumulate in renal cortex and their image intensity increases during the seven days of the experiment. Some metabolites imply minor modification (oxidation, demethylation) of the parent polymyxin, with small reductions of molecular weight. Other metabolites comprise the sequential hydrolysis of the linear lipotripeptide moiety without affecting the cyclic heptapeptide structure. Regarding colistin metabolization, similar byproducts were found. However, one of the metabolites detected involved the partial hydrolysis of cyclic structure in this case (colistin metabolite M5, not shown). ${ }^{198}$ Altogether, this study suggests that in spite of the fact that the majority of kidney's drug metabolizing and detoxification enzymes are located in the proximal tubule, ${ }^{203}$ a significant proportion of polymyxin molecules remain nonmetabolized in renal cortex, what seems to indicate that polymyxins 
are apparently quite stable molecules. In fact, polymyxins accumulate and are reabsorbed in proximal tubules, as we have seen before, and are mainly eliminated by non-renal clearance mechanisms.

\subsection{Biomarkers of nephrotoxicity}

Monitoring of drug-induced kidney injuries is an important issue during drug development. Hence, validation of kidney injury biomarkers is necessary for the clinical application and regulatory issues. In acute kidney injury according to the RIFLE criteria, the determination of plasma and serum creatinine levels remains as a standard of diagnosis. Creatinine is a useful biomarker of kidney damage in patients suffering of stable chronic renal diseases, but it is not convenient in the case of acute kidney disease. More than half of kidney function is lost before AKI is detected by an increase of serum creatinine levels as they are detected 24-48 hours following the initial kidney insult. Colistimethate treatments are often discontinued when minor increments in creatinine levels are detected because of the risk of negative outcomes, even if patients are responding to the antibiotic therapy. Hence, there is a need of novel kidney injury urinary biomarkers that can detect AKI much earlier in order to avoid such decision time lag. Several biomarkers are a subject of study to allow for an early and site-specific prediction of AKI. These indicators can detect initial kidney injury in 4-6 hours. Biomarkers for tubular damage include Kidney Injury Molecule-1 (KIM-1), $\alpha$-Glutathione S-Transferase ( $\alpha$-GST), $\pi$-Glutathione S-Transferase ( $\pi-G S T$ ) and NGAL (Neutrophil Gelatinase Associated Lipocalin). ${ }^{197,204,205}$

KIM-1 is a type I trans-membrane structural glycoprotein present on epithelial cells of the renal proximal tubule. KIM-1 is undetectable in healthy tissue but highly upregulated on the apical surface of proximal tubule epithelial cells after renal injury, i. e. in response to toxic and ischemic injury. The ectodomain shedding of KIM-1 into urine makes it an early and specific biomarker for AKI.

$\alpha-$ GST and $\pi-G S T$ are small cytosolic isoenzymes and members of the GST superfamily. They are primarily involved in cellular detoxification metabolic reactions since they catalyze the conjugation of the reduced form of glutathione ( $L-\gamma-$ glutamyl-Lcysteinyl-glycine) to xenobiotic substrates. They can form up to $2 \%$ of the total cytosolic soluble protein content in the cytoplasm. $\alpha$-GST and $\pi$-GST are immediately released into the urine upon lysis of epithelial cells of the proximal and distal tubule, respectively, converting them in a rapid and sensitive biomarker of AKI. Simultaneous detection of both GST proteins permits discrimination between proximal and distal tubular damage.

NGAL is small extracellular glycoprotein that is characterized by the ability to bind small hydrophobic molecules. NGAL is rapidly upregulated and thus, an early biomarker for the detection of AKI onset in various clinical settings including cardiothoracic surgery, intensive care-unit, and nephropathy.

In a study carried out in rats, KIM-1 and $\alpha$-GST proved to be the most sensitive biomarkers to polymyxin-induced AKI, outperforming current nephrotoxicity standards of care (creatinine and blood urea nitrogen) proving that they are not suitable for detecting nephrotoxicity of polymyxin in this in vivo model. ${ }^{197} \mathrm{NGAL}$ and $\pi$-GST also proved to be useful indicators according to same study. In another in vivo study performed simultaneously in rat, dog and monkey, similar results were obtained for the rat model, with NGAL and KIM-1 capable of detecting AKI (GST biomarkers were not tested). However, in the dog and monkey models, both standard (creatinine and blood urea nitrogen) and more recent biomarkers (NGAL and KIM-1) showed a rapid onset of response. ${ }^{204}$ In this case, though, the added value of urinary NGAL and urinary KIM-1 biomarkers was their selectivity in the localization of the injury within the kidney (proximal and/or distal tubules versus the glomerulus) and can help to discriminate the development of acute kidney injury onset. ${ }^{205}$

\section{Design and development of new polymyxins}

The growing incidence of bacterial resistance in hospitals and healthcare settings, the dry pipeline of new antibiotics, and the fact that polymyxin B and colistin have become last line antibiotics to treat highly drug resistant infections in spite of their toxicity liabilities has led to several worldwide research initiatives in recent years to design and develop new polymyxin analogs. The goals are to improve activity, reduce adverse side effects, mostly nephrotoxicity, and understand the relationship of activity and toxicity with the chemical structure of polymyxins.

First efforts aiming at these objectives started in the 1970s. However, the difficulty in access to reliable peptide synthesis schemes for the preparation of cyclic peptide analogs and the limited knowledge of polymyxin pharmacology reduced the structural and chemical space of explored analogs. ${ }^{14,15,206-210}$ Thus, compounds were generated by acylation or alkylation of Dab residues, or substitution of the $\mathrm{N}$-terminal fatty acid tail sometimes following semisynthetic approaches from truncated versions of polymyxin such as the nonapeptide (PBNP). The view that emerges from this initial background is that amphipathicity in polymyxins is crucial for activity, which includes the charged Dab residues on the one hand and the fatty acid tail and the conserved hydrophobic residues in position 6 and 7 on the other.

Another trend that can be observed relates to the fact that in general, activity in polymyxins runs parallel to toxicity, i. e. more active compounds tend to be more toxic to mammalian cells. Hence, new approaches should be sought to break this activity/toxicity correlation in polymyxins. In the last decade or so, several academic and private teams worldwide got involved in the design and development of novel polymyxin analogs to overcome the drawbacks posed by the natural compounds, that is to say, improve safety and efficacy.

\subsection{Synthetic preparation of polymyxin analogs}

Preparation of novel polymyxin analogs is performed following two main approaches: semisynthesis and total chemical synthesis. The first strategy, semisynthesis, starts from the natural polymyxin product, usually obtained by fermentation procedures. ${ }^{211}$ Although this is a readily accessible and affordable approach, it is curtailed 
by the few possibilities of modifying the cyclic heptapeptide core and normally analogs are reduced to truncation or substitutions in the linear lipotripeptide moiety by enzymatic treatment. Enzymatic hydrolysis of the linear segment of polymyxin gives access to truncated analogs of polymyxin, such as the deacylated decapeptide by means of a polymyxin acylase treatment or to the well-known PBNP by treatment with papain or plant proteinase ficin. ${ }^{202-203}$ Treatment with bromelain enzyme yields polymyxin octapeptide (polymyxin 3-10) while hydrolysis with Nagarse furnishes the heptapeptide (polymyxin 4-10, heptacycle devoid of the N-teminal lipotripeptide). ${ }^{210-211}$ Further elaboration of the polymyxin fragments is possible by means of several selective protection schemes (Boc, tert-butoxycarbonyl, Fmoc, 9-fluorenylmethoxycarbonyl) and strategies as discussed below for each particular case.

Total chemical synthesis of polymyxins generally involves the solidphase methodology using different polymeric supports and protecting groups such as Fmoc, Boc, tBu (tert-butyl), Dde (1-(4,4dimethyl-2,6-dioxocyclohex-1-ylidene)ethyl), Z (benzyloxycarbonyl) or Bzl (benzyl). Below, efforts by private companies and universities devoted to the design and development of polymixins are summarized.

\subsection{CB-182,804 analog}

This was the first synthetic polymyxin B derivative that entered a clinical trial. Originally developed by BioSource Pharm and licensed to Cubist Pharmaceuticals (now part of Merck), it had its N-terminal fatty acyl moiety substituted by an aromatic urea, a 2-chlorophenylaminocarbonyl unit (Figure 5).

The analog CB-182,804 was prepared by semisynthesis from natural polymixin obtained by fermentation. Side chain $\mathrm{Dab}$ amino groups were protected with the Sulfmoc group $\left(\mathrm{HSO}_{3}-\mathrm{Fmoc}\right)$ by reaction with 9-(2-sulfo)fluorenylmethoxycarbonyl- $\mathrm{N}$-hydroxysuccinimide. The resulting penta-Sulfmoc protected polymyxin was treated with a deacylase enzyme (from Actinoplanes ulahensis NRRL 12052) to remove the fatty acid tail and the resulting $\mathrm{N}$-terminal free amino group was reacted with 2-chlorophenylisocyanate to yield the $o$-chlorophenyl urea. Finally, the Sulfmoc protecting groups were removed with piperidine in methanol to obtain the expected polymyxin derivative. ${ }^{212}$

In vitro activity of CB-182,804 showed MIC90 (minimal concentration to inhibit growth of $90 \%$ of bacteria strains) values for E. coli, K. pneumoniae and P. aeruginosa around 2-4 $\mu \mathrm{g} \cdot \mathrm{mL}^{-1}$, similar or slightly higher (less potent) than polymyxin B/colistin. ${ }^{212,213}$ It was also demonstrated to be substantially less toxic in vitro since an $\mathrm{EC}_{50}$ of more than $1000 \mu \mathrm{g} \cdot \mathrm{mL}^{-1}$ was obtained in rat kidney proximal tubule cell cultures whereas $\mathrm{EC}_{50}$ was 318 $\mu \mathrm{g} \cdot \mathrm{mL}^{-1}$ for polymyxin B.

CB-182,804 was tested in eight rodent infection models for efficacy against five Gram-negative pathogens. The efficacy of CB-182,804, for instance, compared favorably to that of polymyxin B, colistin, ciprofloxacin, or imipenem-cilastatin in an in vivo model of $P$. aeruginosa lung infection in neutropenic mice.

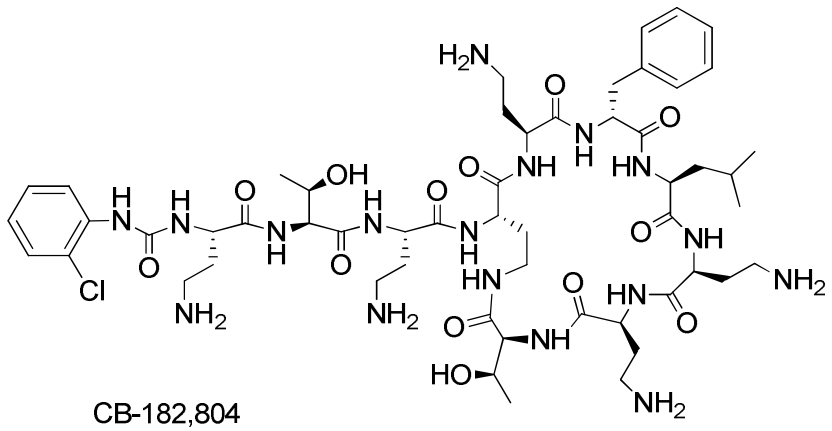

Figure 5: Chemical structure of analog CB-182,804

Similarly, in an A. baumannii thigh infection model in neutropenic mice, it also compared favourably to polymyxin B, colistin or imipenem-cilastatin. Pharmacokinetic features showed differences with respect to polymyxin B, particularly, decreased serum protein binding, and increased plasma clearance and volume of distribution.

Finally, CB-182,804 was tested in cynomolgus monkeys for renal toxicity in a seven-day study. CB-182,804 showed lower nephrotoxicity than polymyxin $B$. Histopathological changes were characterized by minimal to mild degeneration/necrosis $(6.6$ $\mathrm{mg} / \mathrm{Kg} /$ day dose $)^{212}$

There was a mild associated increase in blood urea nitrogen and serum creatinine at higher dose with an increase in severity of histological kidney changes $(9.9 \mathrm{mg} / \mathrm{Kg} /$ day). However, these kidney findings were markedly less than the ones found for polymyxin $B$ when administered at equivalent antimicrobial doses. CB-182,804 progressed into a phase I clinical trial (safety and pharmacokinetics in healthy humans) in February 2009 but in September 2010, it was discontinued. Results have apparently not been published.

\subsection{Pfizer $5 x$ analog}

A series of biaryl and heterobiaryl N-terminal substituted derivatives of polymyxin have been described by Magee and coworkers in Pfizer. ${ }^{214}$ Position 3 that usually contains a Dab amino acid in natural polymyxins was substituted by Dap (L-1,3diaminopropanoic acid) in the analogs (Figure 6). This substitution conferred a higher antimicrobial activity and reduced cytotoxicity in human PTEC cells. Compounds were prepared by solid phase chemical synthesis on a 2-chlorotrityl chloride resin following a Fmoc/tBu/Z/Dde scheme of protection and macrocyclization in solution at high dilution conditions $(0.002 \mathrm{M})$. The heterobiaryl moiety was introduced by acylation reaction of 6-oxo-1-phenyl-1,6dihydropyridine-3-carboxylic acid. Antimicrobial potency of $5 x$ was similar to the one of polymyxin $\mathrm{B}$, as judged by $\mathrm{MIC}_{50}$ and $\mathrm{MIC}_{90}$ (equal or half the value) against susceptible and resistant strains of $P$. aeruginosa, A. baumannii, E. coli and K. pneumoniae. 


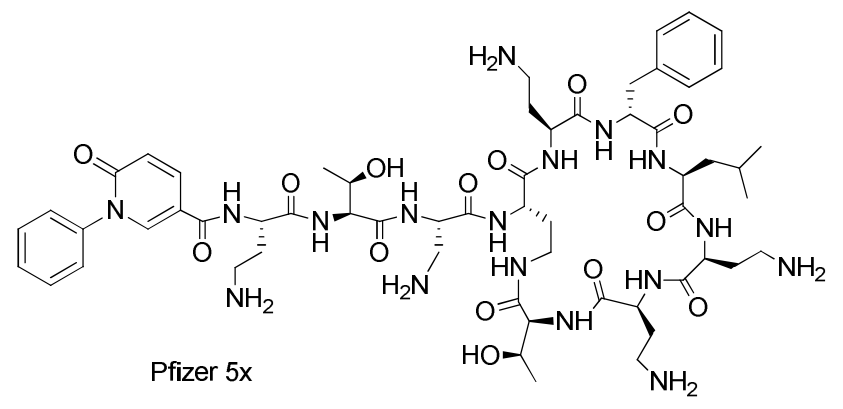

Figure 6: Chemical structure of Pfixer $5 x$ analog

Compound $5 x$ showed improved cytotoxicity in human PTEC cell line $\left(\mathrm{TC}_{50}>100 \mu \mathrm{M}\right)$ in comparison with polymyxin $\mathrm{B}\left(\mathrm{TC}_{50}>22 \mu \mathrm{M}\right)$. A extensive comparative in vivo safety study in rat and dog models was performed. In a seven-day exploratory toxicological study, $5 x$ showed no incidence of necrotic kidney lesions at $4 \mathrm{mg} / \mathrm{Kg} / \mathrm{day}$ dose while polymyxin B yielded necrotic kidney lesions in every animal although graded to be minimal. At twofold dose $(8$ $\mathrm{mg} / \mathrm{Kg} /$ day), $5 \mathrm{x}$ produced no kidney lesions whereas polymyxin $B$ was not even tolerated. However, the dog model proved to be more sensitive to this seven-day exploratory toxicological study. Although at a low dose of $5 \mathrm{mg} / \mathrm{Kg} /$ day was well tolerated, minimal nephrotoxicity signs were already observed in all eight animals tested. At higher doses (11 and $20 \mathrm{mg} / \mathrm{Kg} /$ day) histopathology revealed moderate to marked nephrotoxicity signs. Although the severity of renal lesions was higher for polymyxin $B$, the authors conclude that there was no significant safety margin in dog and further preclinical development was not pursued. This result also demonstrates that the broad in vitro difference in cytotoxicity observed in the PTEC assay in favor of $5 x$ versus polymyxin $B$ did not correlate with the in vivo safety test in dog. Thus, authors conclude that further work needed to be carried out to develop in vitro assays that are able to predict nephrotoxicity in preclinical models, particularly non-rodent ones. ${ }^{214}$

\subsection{Monash FADDI analogs}

The Monash Institute of Pharmaceutical Sciences has also addressed the design of new polymyxin analogs focusing mainly in the hydrophobic domains of the molecule, residues 6 and 7 in the heptapeptide cycle and the fatty acid tail. Compounds were prepared by total chemical synthesis on solid phase. A $\mathrm{Fmoc} / \mathrm{tBu} / \mathrm{Dde}$ scheme of protection on a trityl resin was used. Macrocyclization took place in solution at high dilution conditions. Design of the analogs incorporated new hydrophobic moieties such as octylglycine ( $\mathrm{L}$-2-aminodecanoic acid) and biphenylalanine that resulted in a substantial improvement of in vitro potency against polymyxin resistant isolates. ${ }^{215}$

FADDI-002 and FADDI-003 (Figure 7) yielded MIC of 2-4 $\mu \mathrm{g} \cdot \mathrm{mL}^{-1}$ against polymyxin-resistant strains of $P$. aeruginosa $(32$ to $>32$ $\mu \mathrm{g} \cdot \mathrm{mL}^{-1}$ for polymyxin B) and A. baumannii $\left(2-16 \mu \mathrm{g} \cdot \mathrm{mL}^{-1}\right.$ in front of 8-128 $\mu \mathrm{g} \cdot \mathrm{mL}^{-1}$ for polymyxin B). Against polymyxin sensitive strains, though, FADDI analogs were slightly less potent compared to polymyxin and colistin (MIC 1-4 $\mu \mathrm{g} \cdot \mathrm{mL}^{-1}$ compared to $0.5-2 \mu \mathrm{g} \cdot \mathrm{mL}^{-1}$ ). Activity against Gram-positive bacteria was also reported and resistant strains of Staphylococcus aureus and Enterococcus faecium rendered MIC of 4-16 $\mu \mathrm{g} \cdot \mathrm{mL}^{-1}$.

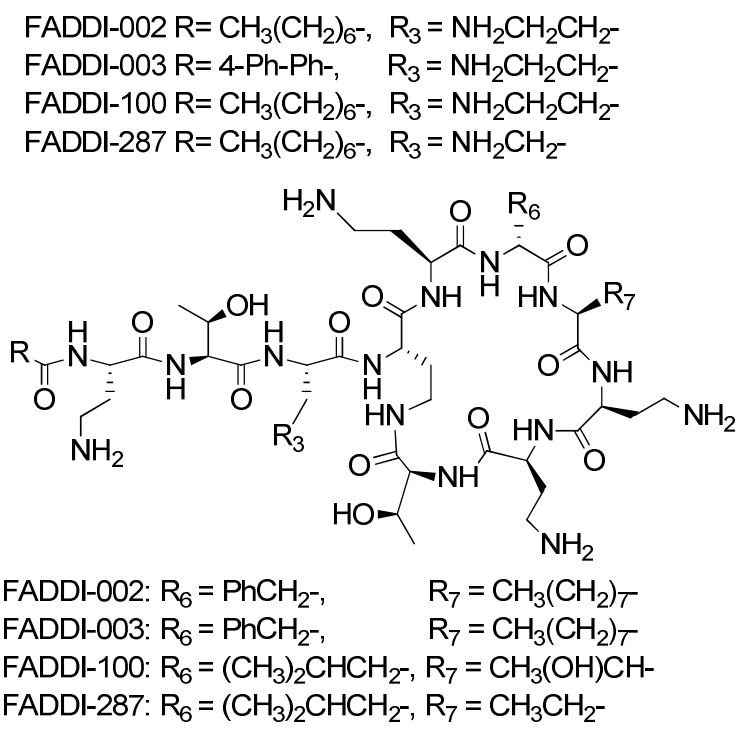

Figure 7: Chemical structure of Monash FADDI analogs

In an in vivo mouse lung infection model, FADDI-002 demonstrated better efficacy than colistin against a polymyxin resistant clinical isolate of $P$. aeruginosa. Regarding safety and tolerability, no adverse effects were detected when a bolus dose of FADDI-002 was administered in rats (intravenous, $0.75 \mathrm{mg} / \mathrm{kg}$ ) and mice (subcutaneous, $40 \mathrm{mg} / \mathrm{kg}$ ). These in vivo preliminary results suggested that the FADDI series had at least a comparable tolerability to polymyxin in rodents. Further exploration led to substitution of position 7 with a threonine (analog FADDI-100), reminiscent of polymyxin $\mathrm{M}$. FADDI-100 rendered a reduction of nephrotoxicity but when tested for potency in a collection of $250 P$. aeruginosa isolates from Rempex-The Medicines Company, a MIC ${ }_{90}$ of $32 \mu \mathrm{g} . \mathrm{mL}^{-1}$ was obtained. ${ }^{216}$ Finally, FADDI-287, a polymyxin E3 analog with position 7 substituted by acid L-2-aminobutanoic (Abu) and Dap in position 3 (like analog $5 x$ reviewed before), provided high potency $\left(\mathrm{MIC}_{90}=1 \mu \mathrm{g} \cdot \mathrm{mL}^{-1}\right.$ in the same collection of $P$. aeruginosa isolates; $\mathrm{MIC}_{90}=0.5 \mu \mathrm{g} \cdot \mathrm{mL}^{-1}$ against $210 \mathrm{~A}$. baumannii isolates). In vivo kidney histopathology in mouse model (dose at 12 $\mathrm{mg}$ base $/ \mathrm{Kg}$, subcutaneously, every $2 \mathrm{~h} \times 6$ ) showed much lower level of nephrotoxicity (mild acute tubular damage with tubular dilation) than polymyxin B (severe acute tubular damage and cortical necrosis). Efficacy test in mice were also encouraging. ${ }^{216}$

\subsection{Queensland analogs}

Cooper's group at the University of Queensland has been involved in the design and preparation of polymyxins and octapeptins. In an article by Gallardo-Godoy and coworkers, they performed a systematic activity-cytotoxicity study involving the preparation of 32 analogs probing eight of the amino acid positions in polymyxin (Figure 8). ${ }^{217}$ Compounds were prepared by solid-phase chemical synthesis following a Fmoc/tBu/allyl scheme of protection on DHP polystyrene resin (3,4-Dihydro-2H-pyran-2-yl-methoxymethyl polystyrene) with side chain anchoring of the C-terminal threonine. Selective Dab modification was also achieved with the use of ivDde (1-(4,4-dimethyl-2,6-dioxocyclohex-1-ylidene)isovaleryl) protecting group when necessary. 


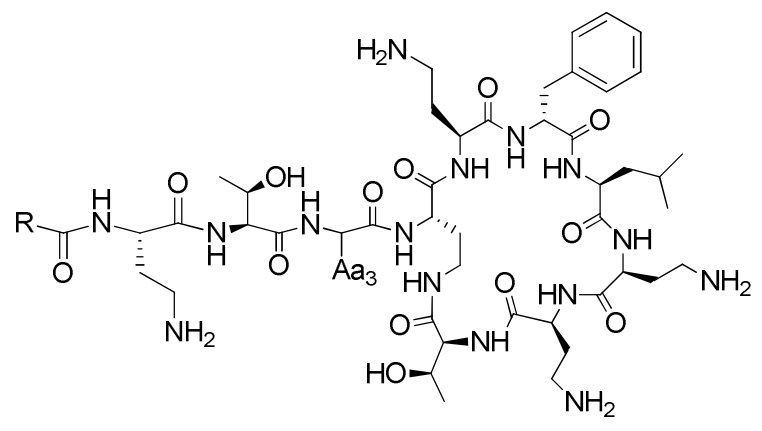

Queensland analog 38, Aa3=Gly, $\mathrm{R}=\mathrm{CH}_{3}\left(\mathrm{CH}_{2}\right)_{6}$

Queensland analog 10, Aa3=Dab, R= 4-Ph-Ph-

Queensland analog 14, Aa3=Dab, R= 4-PhO-Ph-

Figure 8: Chemical structure of University of Queensland analogs (Aa corresponds to the side chain of the indicated amino acid).

MIC results confirmed that more lipophilic side chains at positions $6 / 7$ improved activity against polymyxin-resistant strains but increased cytotoxicity in mammalian cells. Substitution of the Dab residues rendered different behavior depending on the position. Dab-5 and Dab-9 could not be replaced without severely affecting potency, while Dab-8, Dab-3 (substituted by Gly, Thr, D-Dab or DSer, the last two reminiscent of polymyxins $A, D, S$ or $P$ ) and Dab-1 tolerated some substitutions.

Another interesting result of the study was the nephrotoxicity predictive potential of in vitro cytotoxicity tests, an issue that was also discussed at Pfizer's work with analog $5 x .{ }^{214}$ Polymyxin B and colistin cytotoxicity were tested using cell lines HepG2 (hepatocyte carcinoma cells) and HEK293 (embryonic kidney cells). Results showed an apparent lack of toxicity $\left(\mathrm{CC}_{50}>300 \mu \mathrm{M}\right)$ whereas in primary renal cell assay measuring LDH (Lactate Dehydrogenase) and GGT (Gamma Glutamyl Transferase) release, toxicity was clearly evidenced $\left(\mathrm{CC}_{50}\right.$ of $\left.23-177 \mu \mathrm{g} / \mathrm{mL}\right) .{ }^{217}$ An opposite behavior was observed with analog 14 of the series that yielded some toxicity readout in cell lines (287-296 $\mu \mathrm{M})$ while in primary cultures gave a $\mathrm{CC}_{50}$ of $>128 \mu \mathrm{g} / \mathrm{mL}$.

\subsection{Northern Antibiotics analogs}

The team of Vaara at Northern Antibiotics in Helsinki has described a series of polymyxin analogs carrying only the three Dab residues. Compounds are obtained by substituting one or two of the Dab residues by D-Thr, D-Ser or Abu (2-aminobutanoic acid) amino acids, shortening the length of the linear tripeptide moiety of natural polymyxin, or both. The reason for this design lies in the fact that reducing the cationic character by eliminating some of positive charges in the molecule may also lead to a reduction in nephrotoxicity (Figure 9). Compounds of this family have direct antimicrobial activity (NAB739 NAB815) or sensitizing activity with other antibiotics (NAB741 and NAB7061). Analogs were synthesized following conventional solid phase chemical methods using a Fmoc/Bzl/Boc strategy of protection. Cyclization was carried out in solution. ${ }^{16}$ Analog NAB739 is based on polymyxin B but the Nterminal lipotripeptide has been substituted by an octanoyl-ThrDSer segment. NAB739 exhibited good antimicrobial potency against Enterobacteriaceae comparable to the one of polymyxin B.

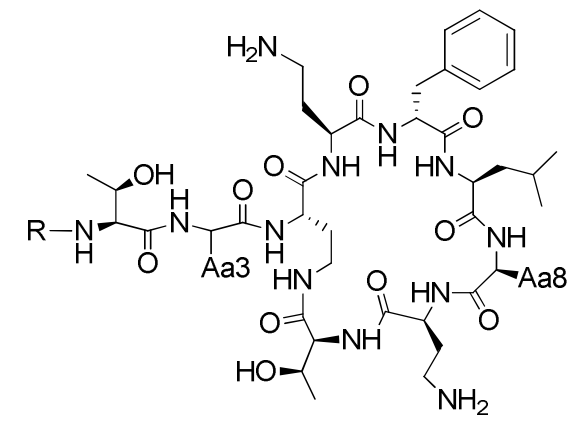

NAB739: $\mathrm{Aa} 3=\mathrm{D}-\mathrm{Ser}, \mathrm{Aa} 8=\mathrm{Dab}, \mathrm{R}=\mathrm{CH}_{3}\left(\mathrm{CH}_{2}\right)_{6}-\mathrm{CO}-$ NAB815: $\mathrm{Aa3}=\mathrm{D}-\mathrm{Thr}, \mathrm{Aa} 8=\mathrm{Abu}, \mathrm{R}=\mathrm{CH}_{3}\left(\mathrm{CH}_{2}\right)_{6}-\mathrm{CONH}-\mathrm{Dab}$ $\mathrm{NAB741}$ Aa3=D-Ser, Aa8=Dab, $\mathrm{R}=\mathrm{CH}_{3}-\mathrm{CO}$ NAB7061: $\mathrm{Aa} 3=\mathrm{Abu}, \quad \mathrm{Aa} 8=\mathrm{Dab}, \mathrm{R}=\mathrm{CH}_{3}\left(\mathrm{CH}_{2}\right)_{6}-\mathrm{CO}-$

Figure 9: Chemical structure of Northern Antibiotics analogs (Aa corresponds to the side chain of the indicated amino acid).

Against E. coli, a concentration of $1 \mu \mathrm{g} / \mathrm{mL}$ of NAB739 inhibited growth in $74.5 \%$ of the strains while polymyxin B inhibited $84.3 \%$ of the strains in the same conditions. Against K. pneumoniae, the values of $\mathrm{MIC}_{90}$ for NAB739 and polymyxin B were 2 and $1 \mu \mathrm{g} / \mathrm{mL}$, respectively. However, NAB739 was not so active against $A$. baumannii ( $\mathrm{MIC}_{90}$ was $8 \mu \mathrm{g} / \mathrm{mL}$, four-fold that of polymyxin B), $P$. aeruginosa ( $\mathrm{MIC}_{90}$ was $16 \mu \mathrm{g} / \mathrm{mL}$, eight-fold) and polymyxinresistant strains. ${ }^{218,219}$ In addition to antibacterial activity, NAB739 displayed sensitizing activity at subinhibitory concentrations, facilitating the penetration of other antibiotics into bacterial cells. Against $A$. baumannii and at a concentration of $0.5 \mu \mathrm{g} / \mathrm{mL}$, NAB739 had synergistic activity and reduced the MIC of rifampicin (from 412 to $0.05-0.1 \mu \mathrm{g} / \mathrm{mL}$ ), clarithromycin (from $6-8$ to $0.5 \mu \mathrm{g} / \mathrm{mL}$ ) and vancomycin (from 256 to $3 \mu \mathrm{g} / \mathrm{mL}$ ). ${ }^{220-222}$ Cytotoxicity of NAB739 proved to be 26 -fold less toxic than polymyxin B in HK-2 (human renal proximal tubular) cells with a $\mathrm{CC}_{50}$ of 337 and $13 \mu \mathrm{g} / \mathrm{mL}$, respectively (colistin's was $45 \mu \mathrm{g} / \mathrm{mL}$ ). In permeabilized (electroporated) LLC-PK1 (porcine renal proximal tubular) cells, polymyxin B induced necrosis at $0.016 \mathrm{mM}(c a 20 \mu \mathrm{g} / \mathrm{mL}$ ), some 8fold lower than that for NAB739. NAB739 was found to be effective in treating $E$. coli peritoneal infection in mice (dosed at $1 \mathrm{mg} / \mathrm{Kg}$ twice). ${ }^{223}$ Recently a new analog NAB815 has been reported, presenting $\mathrm{MIC}_{90}$ values 2 -fold less active against $E$. coli and $K$. pneumoniae and 2 -fold more active against $A$. baumannii than NAB739 (both overall less active than polymyxin B). However, in vivo tests have shown that both NAB815 and NAB739 are better tolerated than polymyxin $B$ in cynomolgus monkeys. In fact, a very significant portion of the dose of NAB815 and NAB739 is excreted into urine within $8 \mathrm{~h}$ after an intravenous infusion. ${ }^{224}$ Further studies will reveal their potential use in the clinic.

Sensitizers, although sometimes devoid of intrinsic antimicrobial activity, retain the ability to permeabilize the outer membrane of Gram-negative bacteria. Polymyxin nonapeptide, a truncated version of polymyxin, is a long time kown paradigm example: it is able to increase susceptibility of $E$. coli and other Enterobacteriacea to some antibiotics, particularly lipophilic and amphiphilic ones, up to 100 -fold times, at even low concentrations $(1-3 \mu \mathrm{g} / \mathrm{mL})$. In addition, since it lacks the $\mathrm{N}$-terminal fatty tail, it is less nephrotoxic. ${ }^{221}$ NAB741 and NAB7061 are analogs of polymyxin B and NAB739, but their $\mathrm{N}$-terminal lipopeptide segments are acetyl- 
Thr-DSer and octanoyl-Thr-Abu, respectively, thus carrying only three positive charges. Both compounds are devoid of direct antibacterial activity but are able to potentiate the 10- to 2000-fold the activity of hydrophobic antibiotics (e.g. macrolides, rifampicin) or large amphiphilic antibiotics (e.g. vancomycin) against Enterobacteriaceae and A. baumannii.

The synergistic activity of NAB7061 has been also proven in vivo, in a mice model of $E$. coli peritoneal infection. Similarly, the combination of NAB7061 (dose $5 \mathrm{mg} / \mathrm{kg}$ ) and erythromycin (10 $\mathrm{mg} / \mathrm{kg}$ ) was also effective. ${ }^{220}$ NAB741 has recently proceeded into preclinical studies, renamed as SPR741 due to an in-license agreement with Spero Therapeutics. ${ }^{225}$ SPR741 showed low nephrotoxicity in vivo in cynomolgus monkey and rat models. Only minimal tubular degeneration was observed at a dose of 60 $\mathrm{mg} / \mathrm{Kg}$.day in monkey. ${ }^{226}$ SPR741 will enter phase I clinical trial (double-blind, placebo-controlled, ascending dose, multi-cohort trial evaluating safety, tolerability and pharmacokinetics) as a potentiator compound. ${ }^{227}$

\subsection{Cantab analogs}

Cantab Anti-Infectives has generated a series of polymyxin analogs focusing on the modification of the $\mathrm{N}$-terminal fragment (Figure 10). Actually, the preparation of compounds was performed from either polymyxin nonapeptide (tetra-Boc protected) obtained from natural polymyxin $B$ by enzymatic treatment with papain or from polymyxin heptapeptide obtained from the penta-Boc protected polymyxin by treatment with savinase in acetonitrile-water mixture. ${ }^{228-231}$ Carboxylic acids to be coupled to the corresponding peptides were obtained from commercial sources or prepared in house. Some analogs (example 50 in Brown's patent) ${ }^{229}$ were also prepared by conventional Fmoc solid-phase chemistry and cyclized in solution. Amino acyl moieties in some analogs had the aminoalkyl side chain of natural Dab 1 embedded in piperazine (analog CA824) or piperidine (CA 900) rings. $\mathrm{MIC}_{90}$ of the three selected analogs CA900, CA824 and CA1049 gave similar or slightly better values than polymyxin $B$ against typical Gram-negatives such as $E$. coli ( 0.125 vs $0.25 \mu \mathrm{g} / \mathrm{mL}$ for polymyxin B), A. baumannii (0.25-2 vs 1 $\mu \mathrm{g} / \mathrm{mL}$ ), P. aeruginosa (1 vs $8 \mu \mathrm{g} / \mathrm{mL}$ ) and $K$. pneumoniae $(0.25 \mathrm{vs}$ $0.5 \mu \mathrm{g} / \mathrm{mL}$ ). CA900 gave particularly good activity against $A$. baumannii, with a $\mathrm{MIC}_{90}$ of $0.125 \mu \mathrm{g} / \mathrm{mL}$ compared to the one of polymyxin $(1 \mu \mathrm{g} / \mathrm{mL})$. All three analogs were less cytotoxic in HK-2 cell cultures $\left(\mathrm{TC}_{50}\right.$ of 64,148 and $167 \mu \mathrm{g} / \mathrm{mL}$ for CA 1049, 824 and 900 , respectively) than polymyxin $\left(\mathrm{TC}_{50}\right.$ of $\left.15 \mu \mathrm{g} / \mathrm{mL}\right){ }^{232}$

In vivo efficacy was carried out with CA824 analog. In neutropenic mouse lung infection models, the antibacterial activity of CA824 proved to be superior to polymyxin B against $A$. baumannii NCTC 13301 and $P$. aeruginosa ATCC 27853 while in a mouse thigh infection model, the antibacterial activity of CA824 against the same A. baumannii strain was comparable. ${ }^{233}$ In January 2017, Spero Therapeutics acquired the assets developed by Cantab AntiInfectives to the owner Pro Bono Bio Ltd to bring the program through pre-clinical and clinical stages of development. ${ }^{234}$

\subsection{Scaffold modified polymyxins}

Throughout this review we have seen that antimicrobial potency in polymyxins tends to correlate with toxicity. Both antimicrobial activity and toxicity side effects in mammalian cells seem to be associated to the amphipathic nature of the molecule (hydrophobic and polycationic).

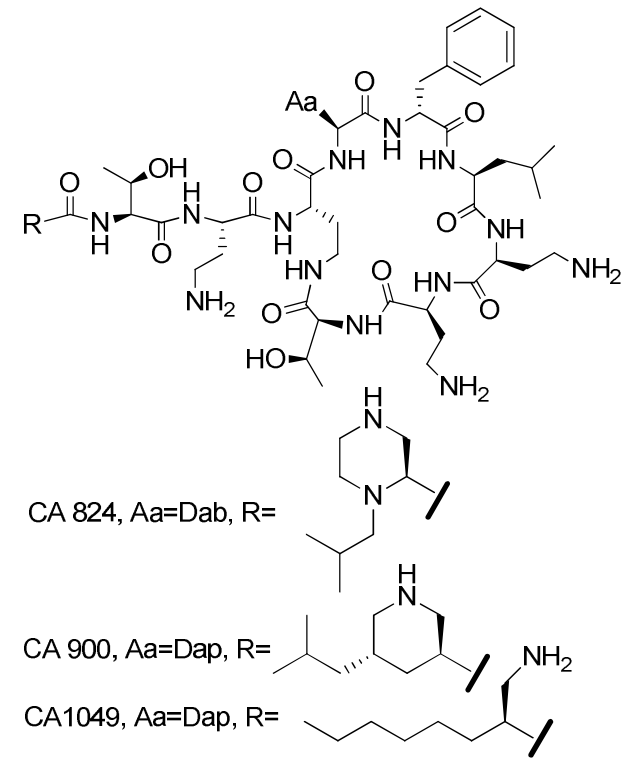

Figure 10: Chemical structures of Cantab analogs

A reasonable approach to break this parallel activity could be the modification of the backbone of the structure, a fact that is also compatible with the substitution of the amino acids of the sequence or the fatty acid to modify the periphery of the molecule. This idea would link to what it is known as soft drug design, which essentially means integrating metabolism considerations into the drug design process. ${ }^{235}$ With this purpose, different chemical units or bonds are introduced into the scaffold to help metabolism to deactivate and detoxify the molecule once it has exerted its desired antimicrobial activity.

In section 10.1., we have seen that polymyxin B and colistin are quite stable molecules. They seem not to be fully metabolized or detoxified in the kidney and accumulate in the renal cortex despite the fact that the majority of kidney's drug metabolizing and detoxification enzymes are located in the proximal tubule (see below). For instance, B- esterases, peptidases and oxidorreductases are localized relatively in high amounts in proximal tubule kidney cells.

Two examples of polymyxin analogs have been recently described in the literature that contain modifications in the peptide backbone designed to aid in the metabolization and prevent accumulation in kidneys, and hence, potentially reduce toxicity: ester-containing ( $\mathrm{i}$. e. depsipeptide) analogs of polymyxin (MicuRx and Barcelona) ${ }^{236,237}$ and disulfide cyclized analogs (Barcelona)..$^{91,237}$

\subsection{MicuRx analogs}

MicuRx has described analogs that incorporate ester, carbamate and phosphate/phosphonate/phosphoramide type of bonds within the N-terminal part of polymyxin (Figure 11 ). ${ }^{236}$ The introduction of esters within polymyxin has been described before. ${ }^{229,238}$ Compounds were prepared by semisynthesis from polymyxin nonapeptide and adding conveniently protected $\mathrm{Fmoc} / \mathrm{tBu}$ amino acids, such as Boc-protected homoisoserine (L-2-hydroxy-4aminobutanoic acid), Fmoc-Dab(Boc)-OH or other acids, by means of standard coupling techniques and reagents. 


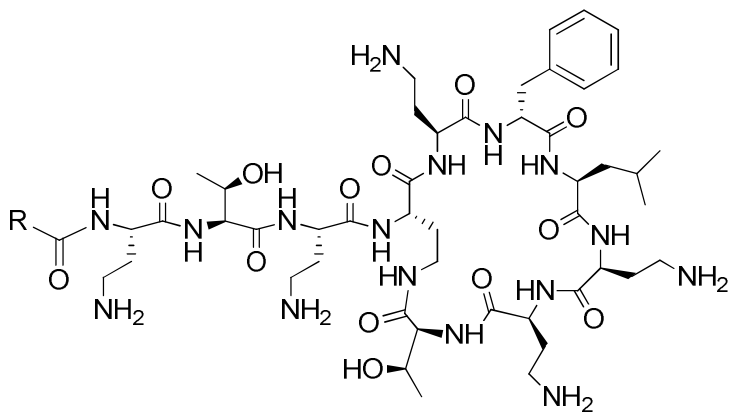

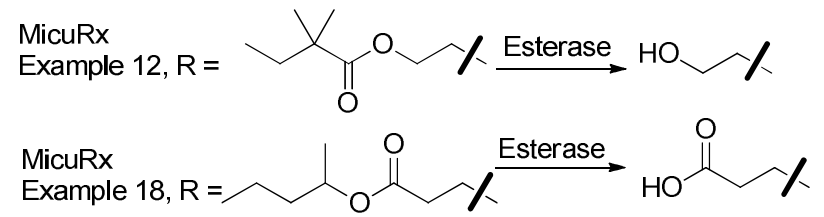

Figure 11: Chemical structure of MicuRx analogs and the hydrolysis products rendered by esterases

Some fifty polymyxin analogs were prepared. Analogs 12 and 18 offered antibacterial activity comparable to polymyxin B and had an ester bond embedded in the fatty acid moiety. They both yielded MIC of $2 \mu \mathrm{g} / \mathrm{mL}$ against $E$. coli (vs $2-4 \mu \mathrm{g} / \mathrm{mL}$ for polymyxin B), 2 and $4 \mu \mathrm{g} / \mathrm{mL}$, respectively against $P$. aeruginosa (vs $2-4 \mu \mathrm{g} / \mathrm{mL}$ ) and 2 and $4 \mu \mathrm{g} / \mathrm{mL}$, respectively against $K$. pneumoniae (vs $2-4 \mu \mathrm{g} / \mathrm{mL}$ ). Cytotoxicity of both analogs in human HK-2 cells was lower $\left(\mathrm{IC}_{50}>\right.$ $200 \mu \mathrm{M})$ than that of polymyxin $\mathrm{B}\left(\mathrm{IC}_{50}=82 \mu \mathrm{M}\right){ }^{236}$

In vivo efficacy of analogs 12 and 18 in E. coli septicemia, $P$. aeruginosa thigh infection, and $P$. aeruginosa lung infection mouse models proved to be comparable to the efficacy results obtained for polymyxin B. A reduction of in vivo nephrotoxicity in rat model was observed for both in comparison with polymyxin B according to the low levels of urine biomarker NGAL (see section 11 before). Finally, stability in human plasma demonstrated that $66-67 \%$ of the original ester compounds 12 and 18 remained non-hydrolized after $1 \mathrm{~h}$ (at 4 h, $31 \%$ and $18 \%)^{236}$

\subsection{Barcelona analogs}

The University of Barcelona has described analogs of polymyxin where the amide bond between the $y$-amino group of Dab4 and the C-terminal carboxy group of Thr10 has been replaced with a disulfide bond (Figure 12). ${ }^{91}$ This replacement is isosteric and implies the substituition of those amino acids with cysteines with an appropriate configuration of the $\alpha$-carbon (L-cysteine in position 4 and D-cysteine in 10). The disulfide link may provide polymyxin analogs with sufficient stability to reach the infectious target in vivo. However, in an eventual accumulation and uptake by renal cells, the disulfide bond may be broken thus opening up the cyclic heptapeptide due to the reducing intracellular environment (reduced glutathione and oxidorreductases) that could facilitate peptide proteolysis and potentially lower renal toxicity. In this respect, detoxification of xenobiotics in kidney is carried out by metabolizing enzymes that are involved, for instance, in the conjugation of glutathione, glucuronic acid, or sulfate. These enzymes, including cytochrome P-450, are mainly localized in kidney's proximal tubules.

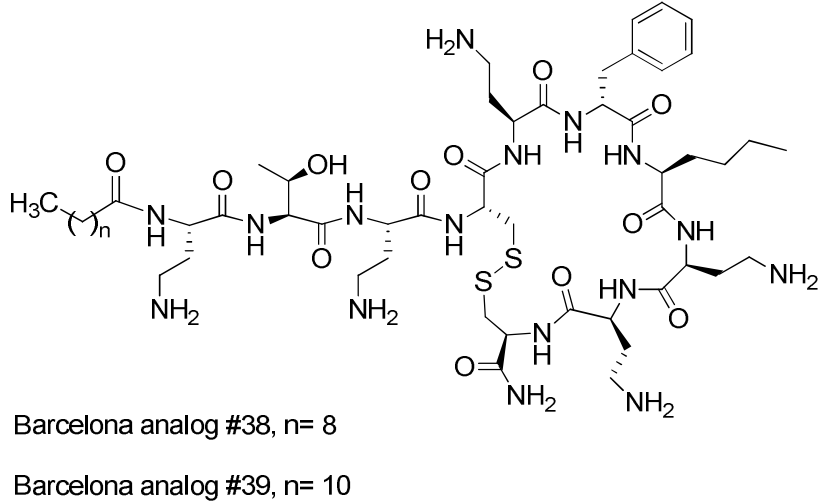

Figure 12: Chemical structure of Barcelona disulfide analogs

In particular, proximal tubular cells exhibit a high activity in glutathione redox cycle enzymes, such as glutathione disulfide reductase, GSH peroxidases, and GSH S-transferase. The intracellular concentration of glutathione in proximal tubular cells is around 2-5 mM whereas in the lumen is at least two orders of magnitude lower. In blood, GSH concentration is around 20 $\mu \mathrm{M}^{203,239,240}$

Furthermore, in an in vivo study in rat dealing with the metabolism and retention of octreotide (a marketed disulfide cyclic peptide also containing two D-amino acids) ${ }^{91}$ in kidney and liver, metabolites of radiolabeled octreotide were shown to be decyclized (linear) products by reduction of the disulfide bond to cysteine and fragmented by hydrolysis of the peptide bonds. ${ }^{241}$

All this data supports the use of the disulfide bond as a tool to modulate the stability of the polymyxin scaffold, facilitate metabolization and reduce accumulation in kidney. In fact, the use of disulfide bonds in drugs should not be seen as exceptional. There are several peptide drugs in the market containing disulfide bonds, such as octreotide, lanreotide or vapreotide (analogs of somatostatin that consist of a disulfide cyclic octapeptide), insulin, linaclotide (for inflammatory bowel syndrome), ziconotide (treatment of pain), pramlintide (for type II diabetes), atosiban (inhibitor of the hormones oxytocin and vasopressin), neseritide (for acute decompensated congestive heart failure), romidepsin (anticancer agent used in cutaneous T-cell lymphoma), and peginesatide (treatment of anemia associated with chronic kidney disease).

Preparation of the analogs was carried out by $\mathrm{Fmoc} / \mathrm{tBu}$ solid phase chemical synthesis. Cyclization by means of disulfide bond formation was performed at high dilution conditions in plain water and dimethylsulfoxide solvent, with no other reagents. Antibacterial activity comparable to polymyxin B was achieved, including resistant and multi-drug resistant strains. Analogs 38 and 39 yielded both MIC of 2 and $4 \mu \mathrm{g} / \mathrm{mL}$, respectively against $E$. coli (vs 1 $\mu \mathrm{g} / \mathrm{mL}$ for polymyxin B), $1 \mu \mathrm{g} / \mathrm{mL}$ against $P$. aeruginosa ( $v s 2 \mu \mathrm{g} / \mathrm{mL}$, polymyxin B). Against resistant strains, Analogs 38 and 39 yielded both MIC of 0.5 and $0.5-1 \mu \mathrm{g} / \mathrm{mL}$, respectively against $E$. coli (vs $0.25-0.5 \mu \mathrm{g} / \mathrm{mL}$ for polymyxin B) and $0.5-4 \mu \mathrm{g} / \mathrm{mL}$ against $P$. 
aeruginosa (vs $1-2 \mu \mathrm{g} / \mathrm{mL}$, polymyxin $\mathrm{B}$ ). ${ }^{91}$ Whatsmore, analog 39 was also active against Gram-positive bacteria $S$. aureus and Enterococcus faecalis, rendering comparable MIC to those of daptomycin and vancomycin. Analog 38 has also demonstrated synergistic and antibiofilm activities with imipenem in a imipenen-resistant strain of $P$. aeruginosa. ${ }^{242}$ Finally, an in vivo acute toxicity test by subcutaneous administration performed on CD-1 mice with analog 38 , the LD50 obtained $(283 \mathrm{mg} / \mathrm{kg}$ ) was clearly superior to the one reported for polymyxin B $(59.5 \mathrm{mg} / \mathrm{kg}){ }^{91}$ Further in vivo tests (pharmacokinetic studies, full toxicity and efficacy) are ongoing and will be reported in due course.

\section{Conclusions and Perspectives}

The emergence of multi-drug resistant Gram-negative bacteria will need of all efforts (antimicrobial chemical design, medicinal chemistry, microbiology, pharmacology, development, clinical studies, rational use of antibiotics) to control this new health challenge.

The research and development of alternative antimicrobial agents is a plausible option to tackle the resistance problem and the "antibiotic discovery void" since the late 1980s. Unfortunately, in the field of polymyxins, only a single candidate, CB-182,804 has progressed to a clinical phase but was finally discontinued. Recently, a new polymyxin molecule, SPR741, has been announced to proceed to clinical phases albeit as a potentiator, not as an antimicrobial per se. ${ }^{227}$ Hence, much effort and new approaches are still needed.

The design, discovery and development of new chemical entities, particularly those with new scaffolds or acting by new mechanism of action will certainly play a fundamental role. Furthermore, advances in the understanding at the molecular level of the mechanism of polymyxin toxicity will provide new insights on how to design new molecules with better therapeutic indexes. In this sense, the incorporation of metabolism considerations into the drug design process is a promising new line. New challenges will rise, though, as these new chemical units need to keep a balance between stability to reach the target and exert the desired antimicrobial activity in vivo and later facilitate metabolization and detoxification of the molecule thus preventing unwanted side effects.

In the short run, natural polymyxin $\mathrm{B} /$ colistin and colistimethate will continue to be used as last-line therapeutic option due to the scarcity of antibiotics against MDR Gram-negative bacteria. Nevertheless, recent pharmacological progress and understanding of the mechanism of action and toxicity in polymyxins provides clinicians with valuable information for optimizing their use in patients (dosage, combinations, nephrotoxicity, biomarkers). ${ }^{243}$ Still a large amount of work is necessary for instance to clarify the advantage of antibiotic combinations with polymyxins. In this sense, clinical trials to compare colistin monotherapy and the combination with meropenem are in progress. ${ }^{150}$

We hope that the next generation of polymyxins will become satisfactory therapeutic tools for the treatment of infections caused by multi-drug resistant bacteria.

\section{Acknowledgements}

We thank the support of the University of Barcelona, Fundació Bosch i Gimpera, Xarxa de Referència en Biotecnologia (XRB, Generalitat de Catalunya) and the European Institute of Innovation and Technology (EIT Health). The authors are members of the ENABLE (European Gram-Negative Antibacterial Engine) consortium (IMI-ND4BB, http://www.imi.europa.eu/content/enable).

\section{References}

1 R. G. Benedict and A. F. Langlykke, J. Bact. 1947, 54, 24

2 P. G. Stansly, R. G. Shepherd and H. J. White, Johns Hopk. Hosp. Bull. 1947, 81, 43

3 G. C. Ainsworth, A. M. Brown and G. Brownlee, Nature, 1947, 160, 263

4 J. A. Orwa, C. Govaerts, R. Busson, E. Roets, A. Van Schepdael and J. Hoogmartens, J. Chromatogr. A, 2001, 912, 369.

5 D. Decolin, P. Leroy, A. Nicolas and P. Archimbault, J Chromatogr Sci., 1997, 35, 557

6 J. A. Orwa, C. Govaerts , R. Busson, E. Roets, A. Van Schepdael and J. Hoogmartens, J Antibiot (Tokyo), 2001, 54, 595

7 J. He, S. Gao, M. Hu, D. S.-L. Chow and V. H. Tam, J Antimicrob. Chemother. 2013, 68, 1104

8 M. E. Falagas, K. N. Fragoulis, , S. K. Kasiakou, G. J. Sermaidis, and, A. Michalopoulos, Int. J. Antimicrob. Agents 2005, 26, 504

9 D. N. Gilbert, R.J. Guidos, H. W. Boucher, G.H. Talbot, B. Spellberg, J. E. Edwards Jr, W. M. Scheld, J. S. Bradley, J. G. Bartlett, Clin. Infect. Dis. 2010, 50, 1081

10 CDC webpage: https://www.cdc.gov/drugresistance/about.html

11 F. Rabanal and Y. Cajal, In: New Weapons to Control Bacterial Growth, pp. 433-452, Springer International Publishing Switzerland 2016, T.G. Villa and M. Viñas (eds.)

12 J. M Sierra, E. Fusté, F. Rabanal, T. Vinuesa and M. Viñas, Expert Opinion on Biological Therapy, 2017, 6, 663-76

13 WHO press release: http://www.who.int/mediacentre/news/releases/2017/bact eria-antibiotics-needed/en/

14 T. Velkov, P. E. Thompson, R. L. Nation and J. Li, J. Med. Chem. 2010, 53, 1898.

15 T. Velkov, K. D.Roberts, R. L. Nation, P. E. Thompson, J. Li, Future Microbiol. 2013, 8, 711

16 M. Vaara, J. Antimicrob. Chemother. 2013, 68, 1213

17 P. Brown and M. J. Dawson, J. Antibiot., 2017, 70, 386

18 W. Raza, W. Yang and Q-R. Shen, Journal of Plant Pathology 2008, 90, 419

19 G. Brownlee, S. R. M. Bushby and E. I. Short Brit. J. Pharmacol. 1952, 7, 170

20 Y. Koyama, A. Kurosawa, A. Tuchiya and K. Takahashi, J. Antibiotics (Tokyo) Ser.B., 1950, 3, 457

$21 \mathrm{~K}$. Hayashi and T. Suzuki, Kyoto University Research information Repository, 1965, http://repository.kulib.kyotou.ac.jp/dspace/bitstream/2433/76067/1/chd043_3_259.pdf

22 D. R. Storm, K. S. Rosenthal and P. E Swanson Ann. Rev. Biochem. 1977, 46, 723

23 N. I. Martin, H. Hu, M. M. Moake, J. J. Churey, R. Whittal, R. W. Worobo and J. C. Vederas, J. Biol. Chem. 2003, 278, 13124

24 B. Niu, J. Vater, C. Rueckert, J. Blom, M. Lehmann, J.-J. Ru, X.H. Chen, Q. Wang and R. Borriss BMC Microbiology, 2013, 13, 137

25 Y. Kimura, E. Murai, M. Fujisawa, T. Tatsuki and F. Nobue, $J$ Antibiot. 1969, 22, 449 
26 J. Shoji, H. Hinoo, Y. Wakisaka, K. Koizumi, M. Mayam and S. Matsuura J. Antibiot., 1977, 30, 1029

27 J. Shoji, T. Kato, H. Hinoo, 1977. J. Antibiot., 1977, 30, 1035

28 J. Shoji, T. Kato, H. Hinoo, 1977. J. Antibiot., 1977, 30, 1042

29 J. Shoji, T. Kato, H. Hinoo, 1977. J. Antibiot., 1977, 30, 427

30 W. L. Parker, M. L. Rathnum, L. D. Dean, M. W. Nimeck, W. E. Brown and E. Meyers, J. Antibiot. 1977, 30, 767

31 S. Terabe, R. Konaka and J. Shoji. J. Chromat. 1979, 173, 313

32 S. A. Cochrane and J. C. Vederas, Medicinal Res. Rev., 2016, 36, 4

33 M. Shaheen, J. Li, A. C. Ross, J. C. Vederas, and S.E. Jensen, Chem. Biol. 2011, 18, 1640

34 T. Velkov, K. D. Roberts and J. Li, Nat. Prod. Rep., 2017,34, 295

35 M.E. Falagas and S.K. Kasiakou, Clin. Infect. Dis., 2005, 40, 1333

36 T. Mogi, Y. Murase, M. Mori, K. Shiomi, S. Omura, M.P. Paranagama and K. Kita, J. Biochem. 2009, 146, 491-499.

37 N. Rastogi, M. C. Potar AND H. L. David, Ann Inst Pasteur Microbiol. 1986, 137, 45

38 B. Zhai and X. Lin, Int. J. Antimicrob Agents, 2013;41:250254

39 J. He, K. R. Ledesma, D. A. Figueroa, T.-P. Lim, D. S. Chow and V. H. Tam. Int. J. Antimicrobial Agents, 2010, 35, 308

40 V. H. Tam, H. Cao, K. R. Ledesma and M. Hu, Antimicrob. Agents Chemother. 2011, 55,4490

41 K. D. Roberts, M. A. K. Azad, J. Wang, A. S. Horne, P. E. Thompson, R. L. Nation, T. Velkov and J. Li, ACS Infect. Dis. 2015, 1, 568

42 Z. Kassamali, R. A. Prince, L. H. Danziger, J. C. Rotschafer, P. R. Rhomberg and R. N. Jones Antimicrob. Agents Chemother. 2015, 59, 7823

43 P. Manchandani, Y. Dubrovskaya, S. Gao and V. H. Tam Antimicrob. Agents Chemother. 2016, 60, 6980

44 S. Sivanesan, K .Roberts, J. Wang, S.-E. Chea, P. E. Thompson, J. Li, R. L. Nation, and T. Velkov J. Nat. Prod., 2017, 80, 225

$45 \mathrm{~A}$. Abdelraouf, J. He, K. R. Ledesma, M. Hu and V. H. Tam, Antimicrob Agents Chemother , 2012, 56, 5724

46 M. E. Falagas, K. Z. Vardakas, http://www.antimicrobe.org/d05.asp

47 M. Barnett, S. R. Bushby and S. Wilkinson, Br. J. Pharmacol. Chemother., 1964,23, 552

48 J. Li, R.W. Milne, R.L. Nation, J.D. Turnidge, T.C. Smeaton and K. Coulthard, J. Antimicrob. Chemother., 2004, 53, 837

49 Bergen, P.J.; Li, J.; Rayner, C.R.; Nation, R.L. Colistin methanesulfonate is an inactive prodrug of colistin against Pseudomonas aeruginosa. Antimicrob. Agents Chemother., 2006, 50, 1953-1958.

50 J. Li, R.W. Milne, R.L. Nation, J.D. Turnidge, and K. Coulthard, Antimicrob. Agents Chemother., 2003, 47, 1364

51 S. Marchand, I. Lamarche, P. Gobin and W. Couet, J Antimicrob Chemother, 2010, 65, 1753

52 R. L. Nation, T. Velkov and J. Li, Clin. Infect. Dis., 2014, 59, 88

53 S. Biswas, J.M. Brunel, J.C. Dubus, M. Reynaud-Gaubert and J.M. Rolain, Expert Rev. Anti.Infect.Ther. 2012, 10, 917

54 European Medicines Agency review of polymyxin-based medicines:

http://www.ema.europa.eu/ema/index.jsp?curl=pages/news and events/news/2014/10/news detail_002194.jsp\&mid= WC0b01ac058004d5c1

55 H.I. Zgurskaya, C.A. López and S. Gnanakaran, ACS Infect. Dis., 2015, 1, 512-522.

56 H. Nikaido, Microbiol. Mol. Biol. Rev., 2003, 67, 593-656.

57 R.M. Epand and R.F. Epand, Biochim. Biophys. Acta, 2009, 1788, 289-294.

58 K. Nishino, F.F. Hsu, J. Turk, M.J. Cromie, M.M. Wösten and E.A. Groisman, Molecular Microbiology, 2006, 61, 645-654.
59 C.R.H. Raetz and C. Whitfield, Annual Review of Biochemistry, 2002, 71, 635-700.

60 M. Schindler and M.J. Osborn, Biochemistry, 1979, 18, 44254430.

61 M.E. Evans, D.J. Feola and R.P. Rapp, The Annals of Pharmacotherapy, 1999, 33, 960-967.

62 R.E. Hancock and D.S. Chapple, Antimicrob. Agents Chemother., 1999, 43, 1317-1323.

63 R.E. Hancock, J Med. Microbiol., 1997, 46, 1-3.

64 R.E. Hancock and R. Lehrer, Trends Biotechnol., 1998, 16, 8288.

65 M. Vaara and T. Vaara, Nature, 1983, 303, 526-528.

66 S.E. Cheah, M.D. Johnson, Y. Zhu, B.T. Tsuji, A. Forrest, J.B. Bulitta, J.D. Boyce, R.L. Nation and J. Li, Sci. Reports, 2016, 6, 26233.

67 M.D. Bruch, Y. Cajal, J.T. Koh and M.K. Jain, J. Am. Chem. Soc., 1999, 121, 11993-12004.

68 P. Pristovsek and J. Kidric, J. Med. Chem. 1999, 42, 46044613.

69 J.J. Meredith, A. Dufour and M.D. Bruch, J. Phys. Chem. B, 2009, 113, 544-551.

70 S. Bhattacharjya, V.I. Mathan and P. Balaram, Biopolymers, 1997, 41, 251-265.

71 J.T. Oh, Y. Cajal, P.S. Dhurjati, T.K. Van Dyk and M.K. Jain, Biochim Biophys Acta, 1998, 1415, 235-245.

72 L. Zhang, P. Dhillon, H. Yan, S. Farmer and R.E.W. Hancock, Antimicrob. Agents Chemother., 2000, 44, 3317-3321.

73 N.P. Mortensen, J.D. Fowlkes, C.J. Sullivan, D.P. Allison, N.B. Larsen, S. Molin and M.J. Doktycz, Langmuir, 2009, 25, 37283733.

74 R. Daugelavicius, E. Bakiene and D.H. Bamford, Antimicrob. Agents Chemother., 2000, 44, 2969-2978.

75 K.A. Brogden, Nat. Rev Microbiol, 2005, 3, 238-250.

76 Z. Yu, W. Qin, J. Lin, S. Fang and J. Qiu, BiomedRes.Int. 2015, $1-11$.

77 J.T. Oh, T.K. Van Dyk, Y. Cajal, P.S. Dhurjati, M. Sasser and M.K. Jain, Biochem. Biophys. Res. Commun., 1998, 246, 619623.

78 M.M. Domingues, M.L. Bianconi, L.R.S. Barbosa, P.S. Santiago, M. Tabak, M.A.R.B. Castanho, R. Itri and N.C. Santos, Biochim. Biophys. Acta, 2013, 1828, 2419-2427.

79 J.T. Oh, Y. Cajal, E.M. Skowronska, S. Belkin, J. Chen, T.K. Van Dyk, M. Sasser and M.K. Jain, Biochim. Biophys. Acta, 2000, 1463, 43-54.

80 Y. Cajal, J. Rogers, O. Berg and M.K. Jain, Biochemistry, 1996, 35, 299-308

81 Y. Cajal, J. Ghanta, K. Easwaran, A. Surolia and M.K. Jain, Biochemistry, 1996, 35, 5684-5695

82 A. Clausell, M.A. Busquets, M. Pujol, M.A. Alsina and Y. Cajal, Biopolymers, 2004, 75, 480-490.

83 H.H. Yim and M. Villarejo, J. Bacteriol., 1992, 174, 3637-3644.

84 A. Liechty, J. Chen and M.K. Jain, Biochim Biophys Acta, 2000, $1463,55-64$.

85 R. Henry, B. Crane, D. Powell, L.D. Deveson, Z. Li, J. Aranda, P. Harrison, R.L. Nation, B. Adler, M. Harper, J.D. Boyce and J. Li, J. Antimicrob. Chemother., 2015, 70, 1303-1313.

86 A. Clausell, F. Rabanal, M. Garcia-Subirats, M.A. Alsina and Y. Cajal, J. Phys. Chem. B, 2006, 110, 4465- 4471.

87 A. Clausell, F. Rabanal, M. Garcia-Subirats, M. A. Alsina and Y. Cajal, Luminescence, 2005, 20, 117-123.

88 A. Clausell, M. Garcia-Subirats, M. Pujol, M.A. Busquets, F. Rabanal and Y. Cajal, J. Phys. Chem. B, 2007, 111, 551-563.

89 A. Grau-Campistany, A. Manresa, M. Pujol, F. Rabanal and Y. Cajal, Biochem. Biophys. Acta, 2016, 1858, 333-343.

90 A. Grau-Campistany, M.Pujol, A.M. Marqués, A. Manresa, F. Rabanal and Y. Cajal, Colloids and Surfaces A, 2015, 480, 307317. 
91 F. Rabanal, A. Grau-Campistany, X. Vila-Farrés, J. GonzalezLinares, M. Borràs, J. Vila, A. Manresa and Y. Cajal. Sci. Rep., 2015, 5, 1-11.

92 Z. Z. Deris ZZ, J. Akter, S. Sivanesan, K.D. Roberts, P.E. Thompson, R.L. Nation, J. Li and T. Velkov, The Journal of Antibiotics, 2014, 67, 147-151.

93 J.N. Blaza, H.R. Bridges, D. Aragão, E.A. Dunn, A. Heikal, G.M. Cook, Y. Nakatani and J. Hirst, Sci. Rep., 2017, 7, 4016540176.

94 D.C. LaPorte, K.S. Rosenthal and D.S. Storm, Biochemistry, 1977, 16, 1642-1648.

95 G.H. De Zoysa and V. Sarojini, ACS Appl. Mater. Interfaces, 2017, 9, 1373-1383.

96 M. Rhouma, F. Beaudry, W. Thériault and A. Letellier, Front. Microbiol., 2016, 7, 1789.

97 Z.Z. Deris, J.D. Swarbrick, K.D. Roberts, M.A. Azad, J. Akter, A.S. Horne, R.L. Nation, K.L. Rogers, P.E. Thompson, T. Velkov and J. Li, Bioconjug. Chem., 2014, 25, 750-760.

98 T.R. Sampson, X. , M.R. Schroeder, C.S. Kraft, E.M. Burd and D.S. Weiss, Antimicrob. Agents Chemother., 2012, 56, 5642-5649.

99 P.I. Pereira Ramos, M.G. Flores Custódio, G. Quispe Saji, T. Cardoso, G. Lucchetti da Silva, G. Braun, W.M.B.S. Martins, R. Girardello, A.T. Ribeiro de Vasconcelos, E. Fernández, A.C. Gales and M.F. Nicolás, BMC Genomics, 2016, 17, 447-462.

100 J.A. Imlay, Nat. Rev. Microbiol., 2013, 11, 443-454.

101 S. Pournaras, A. Poulou, K. Dafopoulou, Y.N. Chabane, I. Kristo, D. Makris, J. Hardouin, P. Cosette, A. Tsakris and E. Dé, Antimicrob Agents Chemother.,2014, 58, 828-32.

102 A. O. Olaitan, S. Morand and J. M. Rolain, Frontiers in Microbiology 2014, 5, 643.

103 J. M. A. Blair, M. A. Webber, A. J. Baylay, D. O. Ogbolu and L. J. V. Piddock. Nature Microbiology, 2015, 13, 43

104 L. Poirel, A. Jayol, and P. Nordmann. Clin. Microbiol. Rev. 2017, 30, 557-596

105 K. Jeannot, A. Bolard, and P. Plésiat_Int. J. Antimicrobial Agents 2017, 49, 526-535

106 M. Vaara, T. Vaara, M. Jensen, I. Helander, M. Nurminen, E. Th. Rietschel, P.H. Mäkelä, FEBS Lett., 1981, 129, 145-149

107 Y.-Y. Liu, Y. Wang, t. M. Walsh; L-X. Yi et al. Lancet Infectious Diseases, 2016, 16, 161-168

108 A. P Magiorakos, A. Srinivasan, R. B. Carey, Y. Carmeli, M. E. Falagas, C. G. Giske, et al. Clin Microbiol Infect. 2012, 18, 26881.

109 E. K. Schneider, F. Reyes-Ortega, T. Velkov and J. Li Essays in Biochem. 2017, 61 115-125

110 M. F. Richter, B. S. Drown, A. P. Riley, A. Garcia, T. Shirai, R. L. Svec and P. J. Hergenrother, Nature, 2017, published online 10 May 2017, doi:10.1038/nature22308

111 O. Zusman, t, Avni, I. Leibovici, A. Adler, I. Friberg, t. Stergiopoulou T, Y. Carmeli, P. Mical Antimicrob. Agents Chemother., 2013, 57, 5104-5111

112 J. He, k. Kaniga, A. S. Lynch, R. K. Flamm, T. A. , Diagn. Microbiol. Infect. Dis., 2012, 74, 417-419

113 B. W. Gunderson, K. H. Ibrahim, L. B. Hovde, T. L. Fromm, M. D. Reed and J. C. Rotschafer, Antimicrob. Agents Chemother. 2003, 47, 905-9

114 B. B. D'Souza, S. R. Padmaraj, P. D. Rekha, R. C. Tellis, S. Prabhu and P. Pothen, Microb. Drug Resist, 2014, 20, 550-4

115 G. Samonis, S. Maraki, D. E. Karageorgopoulos, E. K. Vouloumanou and M. E. Falagas ME. Eur. J. Clin. Microbiol. Infect. Dis., 2012, 31, 695-701

116 G. A. Pankuch, H. Seifer, P. C. and Appelbaum., Diagn. Microbiol. Infect. Dis., $2010,67,191-7$

117 X Di, R. Wang, B. Liu, Z. Xin, N. Wentao, W. Jin, L Beibei, C. Yun and L. Youning, J. Antibiot. (Tokyo), 2015, 68, 551555
118 M. Berditsch, T. Jäger, N. Strempel, T. Schwartz, J. Overhage, A. S. Ulrich, Antimicrob Agents Chemother. 2015, 59,5288 .

119 M. Berditsch, H.Lux, O. Babii, S. Afonin and A. S. Ulrich Pharmaceuticals 2016, 9, 56

120 C. Tascini, G. Gemignani, S. Ferranti, E. Tagliaferri, A. Leonildi, A. Lucarini and F. Menichetti, J Chemother, 2004, 16, 282-287

121 R. Sharma, S. Patel, C. Abboud, J. Diep, N. S. Ly, J. M. Pogue, K. S. Kaye. J. Li, G. G. Rao, Int. J. Antimicrobial Agents, 2017, 49, 224-232.

122 M. Souli, I. Galani, S. Boukovalas, m. G. Gourgoulis, Z. Chryssouli, K. Kanellakopoulou, T. Panagea and H. Giamarellou, Antimicrob. Agents Chemother., 2011, 55, 2395-7

123 M. S. Albur, A. Noel, K. Bowker and A. McGowan Int. J. Antimicrobial Agents, 2015, 46, 560-567

124 C. Vidaillac, L. Benichou and R. E. Duval, , Antimicrob. Agents Chemother., 2012, 56, 4856-61.

125 G. A. Pankey and D. S. Ashcraft, Diagn. Microbiol. Infect. Dis., 2011, 70, 561-4.

126 P. Galbani, D. Lombardo, R. E. Lewis, M. Mercuri, S. Bonora, M. P. Landini and S. Ambretti, J. Antimicrob. Chemother., 2014, 69, 1856-65

127 N. Abdul Rahim, S. E. Cheah, M. D. Johnson, H. Yu, h. E. Sidjabat, J. Boyce, et al. J. Antimicrob. Chemother., 2015, 70, 2589

128 I. Rodríguez-Avial, I. Pena, J. J. Picazo, C. Rodríguez-Avial and E. Culebras. Int.J. Antimicrobial Agents , 2015, 46, 616-21

129 T. Tängdén, R. A. Hickman, P. Forsberg, P. Lagerbäck, C. G. Giske and O. Cars, Antimicrobial Agents Chemother., 2014, 58, 1757-1762.

130 M. Bassetti, E. Repetto, E. Righi et al. J. Antimicrob. Chemother., 2008, 61,417-420

131 S. Motaouakkil, B. Charra, A. Hachimi, H. Nejmi, A. Benslama, N. Elmdaghri, H. Belabbes and M.Benbachir M. J. Infect., 2006, 53, 274-278

132 G. M. Hogg, J. G. Barr and C. H. Webb. J. Antimicrob. Chemother., 1998, 41, 494-495

133 H. J. Lee, P. J. Bergen, J. B. Bulitta, B. Tsuji, A. Forrest, R. L. Nation, et al. Antimicrob Agents Chemother. 2013, 57, 3738-45.

134 N. Petrosillo, E. loannidou and M. E. Falagas. Clin. Microbiol.Infect., 2008, 14, 816-827

135 D. W. Wareham, N. C. Gordon and M. Hornsey., J Antimicrob. Chemother. 2011, 66, 1047-51.

136 N. C. Gordon, K. Png and D. W. Wareham, Antimicrob. Agents Chemother. 2010, 54, 5316-22.

137 M. Hornsey and D. W. Wareham, Antimicrob. Agents Chemother. 2011, 55, 3534-7

138 M. Hornsey, C. Longshaw, L. Phee and D. W. Wareham, Antimicrob. Agents Chemother. 2012, 56, 3080-5.

139 M. Hornsey, L. Phee, C. Longshaw and D. W. Wareham, Int J Antimicrob Agents, 2013, 41, 285-7.

140 W. Ni, X. Shao, X. Di, J. Cuiand R. Y. Wang, Int. J. Antimicrob. Agents. 2015, 45, 8-18

141 B. Fan, J. Guan, X. Wang and Y. Cong, PLoS ONE. 2016, 11, e0157757

142 F. Timurkaynak, F. Can, O. K. Azap, M. Demirbilek, H. Arslan and S. O. Karaman. Int. J. Antimicrob. Agents, 2006, 27, 224-228

143 C. H. Lee, Y. F. Tang YF, L. H. Su LH, C. C. Chien, CC, J. W. Liu, Microb. Drug Resis.t, 2008, 14, 233-237

144 D. R. Bowers, H. Cao, J. Zhou, K. R. Ledesma, D. Sun, O. Lomovskaya, et al. Antimicrob Agents Chemother. 2015, 59, 2720-5.

145 H. Yang, G. Chen, L. Hu, Y. Liu,, J. Cheng, H. Li , Y. Ye and J. Li, Int. J. Antimicrob. Agents, 2015, 45, 188-191 
146 D. Field, N. Seisling, P. D. Cotter, R. P. Ross, C. Hill, Frontiers in Microbiol.. 2016, 7, 1713.

147 W. Liang, X. .F Liu, J. Huang, D. M. Zhu, j. Li and J. Zhang, BMC Infect. Dis. 12011, 11, 109

148 J. R. Lenhard, R. L. Nation, and B. T. Tsuji, 2016, 48, 607613.

149 X. Zhang, F. Guo, H. Shao, X. Zheng, Journal of Infection 2017, 74, 118-130

150 Trial for the Treatment of Extensively Drug-Resistant Gram-negative

https://clinicaltrials.gov/ct2/show/NCT01597973 https://clinicaltrials.gov/ct2/show/NCT01732250

151 S. N. Schwartz, G. Medoff, G. S. Kobayashi, C. N. Kwan, and D. Schlessinger Antimicrob. Agents Chemother., 1972, 2, 36-40

152 B. Zhai , H. Zhou, L. Yang, J. Zhang, K. Jung, C.-Z. Giam, X. Xiang and X. Lin J. Antimicrob. Chemother., 2010, 65, 931-938

153 N.A. Moneib, J. Chemother., 1995, 7, 525-529.

154 S. Pietschmann, K. Hoffmann, M. Voget and U. Pison. Veterinary Res. Comm.. 2009, 33, 489-505.

155 R. Ben-Ami, R. E. Lewis, J. Tarrand, K. Leventakos and D. P. Kontoyiannis, Antimicrob. Agents Chemother. 2010, 54 484-490.

156 H. Schemuth, S. Dittmer, S., M. Lackner, L. Sedlacek, A. Hamprecht, E. Steinmann, J. Buer, P. M. Rath, J. Steinmann, Mycoses, 2013, 56, 297-303.

157 T. P. Venturini, L. Rossato, F. Chassot , J. Tairine Keller, F. Baldissera Piasentin, J. Morais Santurio, S. Hartz Alves, J. Med. Microbiol, .2016, 65, 770-4.

158 L-H Hsu, H-F Wang, P-L Sun, F-R Hu, and Y-L Chen_Int. J. Antimicrobial Agents, 2017, in press

159 E. K. Adams, D. S. Ashcraft and G. A. Pankey, Am. J. Med. Sci. 2016, 35, 265-70.

160 G. Pankey, D. Ashcraft, H. Kahn and A. Ismail, Antimicrob. .Agents Chemother. 2014, 58, 5795-5800

161 U. Zeidler, M.E. Bougnoux, A. Lupan, A. et al. J Antimicrob Chemother. 2013, 68, 1285-1296

162 S. M. Ribeiro, M. R. Felício, E. Vilas Boas , S. Gonçalves, F. F. Costa , R. Perumal Samy, N. C. Santos and O. L. Franco, Pharmacology \& Therapeutics , 2016,160 133-144

163 R. Gopal, Y. G. Kim, J. H. Lee, S. K. Lee, J. D. Chae, B. K. Son, C. H. Seo and Y. Park, Antimicrob. Agents Chemother., 2014, 58, 1622-1629

164 J. Lora-Tamayo, O. Murillo, P.J. Bergen, R.L. Nation, A. Poudyal, X. Luo, H.Y. Yu, J. Ariza, and J. Li, J. Antimicrob. Chemother. 2014, 69, 2434-2442

165 C. de la Fuente-Núñez, F. Reffuveille, E. F. Haney, S. K. Straus and R. E. Hancock. PLoS Pathog. 2014,10, e1004152

166 K. Tarquinio, K. Confreda, J. Shurko and K. LaPlante, Antimicrobial Agents and Chemotherapy. 2014, 58, 17231729.

167 R.Rosales-Reyes, M.D. Alcántara-Curiel, M.D. JarilloQuijada, C. Gayosso-Vázquez, M. Morfin-Otero, E. RodríguezNoriega and J. Santos-Preciado, Chemotherapy, 2015-2016, 61, 8-14

168 I. d'Angelo, B. Casciaro, A. Miro, F Quaglia, M. L. Mangoni and F. Ungaro, Colloids Surf. B 2015, 135, 717-725.

169 C. de la Fuente-Núñez, M. H. Cardoso, C. E. de Souza, O. L., Franco and R. E. W. Hancock, Biochim. Biophys. Acta, 2016, 1858, 1061-1069

170 W. Wei, H. Yang, L. Hu, Y. Ye and J. Li - Journal of Microbiology, Immunology and Infection, 2015, xx, 1e10, [Epub ahead of print]

171 D. S. Akajagbor, S. L. Wilson, K. D. Shere-Wolfe, P. Dakum, M. E. Charurat and B. L. Gilliam, Clin Infect Dis. 2013, 57, 1300-3.

172 M. E. Falagas and S. K. Kasiakou Critical Care. 2006, 10(1):R27. doi:10.1186/cc3995.
173 M. E. Falagas, P. I. Rafailidis, E. Ioannidou, V. G. Alexiou, D. K. Matthaiou, D. E. Karageorgopoulos, A. Kapaskelis, D. Nikita, A. Michalopoulos, Int. J. Antimicrob. Agents 2010, 35, 194-199

174 J. A. Kwon, J. E. Lee, W. Huh, K. R. Peck, Y. G. Kim, D. J. Kim, H. Y. Oh. Int. J. Antimicrob. Agents 2010, 35, 473-477

175 J. D. Hartzell, R. Neff, J. Ake, R. Howard, S. Olson, K. Paolino, M. Vishnepolsky, A. Weintrob and G. Wortmann, Clin. Infect. Dis. 2009, 48, 1724-1728

176 H. Ko, M. Jeon, E. Choo, E. Lee, T. Kim, J. B. Jun and H. W. Gil, Nephron. Clin. Pract. 2011, 117, c284-c288

177 A. Trifi, S. Abdellatif, F. Daly, K. Mahjoub, R. Nasri, M. Oueslati, R. Mannai, M. Bouzidi and S. Ben Lakhal, Chemotherapy, 2016, 61, 190-6.

178 C. A. DeRyke, A. J. Crawford, N. Uddinand M. R. Wallace, Antimicrob. Agents Chemother., 2010, 54, 4503-4505

179 C. Dai, S. Tang, S. Deng, S. Zhang, Y. Zhou, T. Velkov, J. Li, and X. Xiao. Antimicrob Agents Chemother., 2015, 59, 579585

180 L. Dalfino, F. Puntillo, M. J. Ondok, A. Mosca, R. Monno, S. Coppolecchia, M. L. Spada, F. Bruno and N. Brienza, Clin Infect Dis. 2015, 61, 1771-7

181 R. Sirijatuphat, S. Limmahakhun, V. Sirivatanauksorn, R. L. Nation, J. Li and V. Thamlikitkul. Antimicrob Agents Chemother. 2015, 59, 3224-3232

182 M. S. Oliveira, G. V. Prado, S. F. Costa, R. S. Grinbaum and A. S. Levin AS. Polymyxin B and colistimethate are comparable as to efficacy and renal toxicity. Diagn. Microbiol. Infect. Dis. 2009, 65,431-4

183 K. Z. Vardakas and M.E. Falagas. Int. J. Antimicrob. Agents, 2017, 49, 233-238

184 K. Phe, Y. Lee, P. M. McDaneld, N. Prasad, T. Yin, D. A. Figueroa, W. L. Musick, J. M. Cottreau, M. Hu and V. H. Tam Antimicrob. Agents Chemother. 2014, 58, 2740-6.

185 A. P. Zavascki and R. L. Nation, Antimicrob. Agents Chemother., 2017, 61, pii: e02319-16

186 S.-E. Cheah, J. Li, B.T. Tsuji, A. Forrest, J. B. Bulitta and R. L. Nation Antimicrob. Agents Chemother. 2016 vol. 60 no. 7 3921-3933

187 MdF Fernandes Vattimo, M. Watanabe, C. Dezoti da Fonseca, L. Barros de Moura Neiva, E. Andrade Pessoa and F. Teixeira Borges, Plos One 2016, 11, e0161057

188 L. S. McCoy, K. D. Roberts, R. L. Nation, P. E. Thompson, T. Velkov, J. Li, and Y. Tor, Chembiochem, 2013, 14, 20832086.

189 T. Suzuki, H. Yamaguchi, J. Ogura, M. Kobayashi, T. Yamada and K. Iseki, Antimicrob. Agents Chemother., 2013, $\mathbf{5 7}, 6319-6324$.

190 S. K. Moestrup, S. Cui, H. Vorum, C. Bregengård, S. E. Bjørn, K. Norris, J. Gliemann and E. I. Christensen, J. Clin. Invest. 1995, 96, 1404-1413.

191 J. M. Lopez-Novoa, Y. Quiros, L. Vicente, A. I. Morales, and F. J. Lopez-Hernandez, Kidney Int. 2011, 79, 33-45.

192 P. Manchandani, J. Zhou, J. T. Babic, K. R. Ledesma, L. D. Truong and V. H. Tam, Antimicrob. Agents Chemother. 2017 AAC-02391. Posted Online 17 January 2017

193 A. P. Zavascki, L. Z. Goldani, G. Y. Cao, S. V. Superti, L. Lutz , A. L. Barth, F. Ramos, M. M. Boniatti, R. L. Nation and J. Li, Clin. Infect. Dis. 2008, 47, 1298-1304

194 M. A. K. Azad, b. A. Finnin, A. Poudyal, K. Davis, J. Li, p. A. Hill, R. L. Nation, T. Velkov and J. Li, Antimicrob. Agents Chemother., 2013, 57, 4329-4335

195 J. Li, K. Coulthard, R. Milne, R.L. Nation, S. Conway, D. Peckham, C. Etheringtonand J. Turnidge, J. Antimicrob. Chemother., 2003,52, 987-992.

196 Li, J.; Milne, R.W.; Nation, R.L.; Turnidge, J.D.; Smeaton, T.C.; Coulthard, K. J. Antimicrob. Chemother., 2004, 53, 837 840 
197 N. D. Keirstead, M. P. Wagoner, P. Bentley, M. Blais, C. Brown, L. Cheatham, P. Ciaccio, Y. Dragan, D. Ferguson, J. Fikes, M. Galvin, A. Gupta, M. Hale, N. Johnson, W. Luo, F. McGrath, M. Pietras, S. Price, A. G. Sathe, j. C. Sasaki, D. Snow, R. L. Walsky and G. Kern, Toxicol Sci. 2014, 137, 27891

198 A. Nilsson, R. J. A. Goodwin, J. G. Swales, R. Gallagher, H. Shankaran, A. Sathe, S. Pradeepan, A.Xue, N. Keirstead, J. C. Sasaki, P. E. Andren and A. Gupta, Chem. Res. Toxicol. 2015, 28, $1823-1830$

199 T. Velkov, B. Yun, E. K. Schneider, M. A. K. Azad, O. Dolezal, F. C. Morris, R. L. Nation, J. Wang, K. Chen, H. H. Yu, L. Wang, P. E. Thompson, K. D. Roberts and J. Li, ACS Infectious Diseases 2016, 2, 341-351

200 M. A. K. Azad, K. D. Roberts, H. H. Yu, B. Lu, A. V. Schofield, S. A. James, D. L. Howard, R. L. Nation, K. Rogers, M. D. de Jonge, P. E. Thompson, J. Fu, T. Velkov, and J. Li, Anal. Chem. 2015, 87, 1590-1595.

201 B. Yun, M. A. K. Azad, C. J. Nowell, , R. L. Nation, P. E. Thompson, K. D. Roberts, T. Velkov and J. Li, Antimicrob. Agents Chemother. 2015, 59, 7489-7496.

202 B. Yun, M. A. K. Azad, J. Wang, R. L. Nation, P. E. Thompson, K. D. Roberts, T. Velkov and J. Li, J. Antimicrob. Chemother. 2015, 70, 827-829.

203 E. A Lock and C. J. Reed, Toxicologic Pathology, 1998, 26, 18-25

204 D. Burt, S. J. Crowell, D. C. Ackley, T. V. Magee and J. Aubrecht, Drug Chemical Toxicol., 2014, 37, 204-212,

205 J. V. Bonventre, V. S. Vaidya, R. Schmouder R, P. Feig and F. Dieterle, Nat Biotechnol 2010, 28, 436-440.

206 J. Alsina, F. Rabanal , E. Giralt and F. Albericio, Tetrahedron Lett., 1994, 35, 9633

207 J. Alsina , F. Rabanal, C. Chiva, E. Giralt and F. Albericio, Tetrahedron 1998, 54, 10125

208 S. Chihara, M. Yahata, T. Tobita and Y. Koyama, Agric. Biol. Chem. 1974, 38, 521-529

209 S. Chihara, T. Tobita, M. Yahata, A. Ito and Y. Koyama, Agric. Biol. Chem. 37, 2455-63 (1973).

210 T. Suzuki, K. Hayashi and K. Fujikawa, J. Biochem, 1963, 54, 412

211 Y. Kimura, H. Matsunaga, M. Vaara, J Antibiot (Tokyo). 1992, 45, 742-9

212 R. A. Leese patent aplication WO2010075416

213 J. Quale, N. Shah, P. Kelly, E. Babu, M. Backer, G. RosasGarcia, J. Salamera, A. George, S. Bratu, D. Landman, Microb. Drug Resist. 2012, 18, 132-136

214 T. V. Magee, M. F. Brown, J. T. Starr, D. C. Ackley et al.. J. Med. Chem., 2013, 56, 5079-5093

215 T. Velkov, K. D. Roberts, R. L. Nation, J. Wang, P. E. Thompson, and J. Li, ACS Chem. Biol. 2014, 9, 1172-1177

$2162^{\text {nd }}$ International Conference on Polymyxins, September, 22-24, 2015 San Diego, California, USA http://www.isap.org/images/polymyxinconference2015/Day-2-slides-session-8-2.pdf

217 A. Gallardo-Godoy, C. Muldoon, B. Becker, A. G. Elliott, L. H. Lash, J. X. Huang, J. X. Huang, M. S. Butler, R. Pelingon, A. M. Kavanagh, S. Ramu, W. Phetsang, M. A. T. Blaskovich and M. A. Cooper, J. Medicinal Chem., 2016, 59, 1068-1077

218 M. Vaara, J. Fox, G. Loidl, O. Siikanen, J. Apajalahti, F. Hansen, N. Frimodt-Møller, J. Nagai, M. Takano and T. Vaara Antimicrob. Agents Chemother. 2008, 52, 3229-36.

219 M. Vaara, H. S. Sader, P. R. Rhomberg, R. N. Jones and T. Vaara, J. Antimicrob Chemother. 2013, 68, 636-9.

220 M. Vaara, O. Siikanen, J. Apajalahti, N. Frimodt-Møller, and T. Vaara, J. Antimicrob Chemother. 2010, 65, 942-5.
221 M. Vaara, Microbiol. Rev. 1992, 56, 395-411.

222 M. Vaara and T. Vaara, patent application WO2016/113470

223 C. Vingsbo Lundberg, T. Vaara, N. Frimodt-Møller and M. Vaara, J. Antimicrob Chemother. 2010, 65, 981-5.

224 M. Vaara, T. Vaara and J. M. Tyrrell, Peptides, 2017, 91, 8-12

225 D. Erickson, Start Up, 2015 (online journal) https://sperotherapeutics.com/wpcontent/uploads/2016/02 /StartUP_Spero_Therapeutics_SU1512.pdf https://scrip.pharmamedtechbi.com/SC092696/SperoTherapeutics--Remodeling-Antibiotics

226 S. Coleman, M. Bleavins, T. Lister, M. Vaara, and T. R. Jr Parr, The Assessment of SPR741 for Nephrotoxicity in Cynomolgus Monkeys and Sprague-Dawley rats. Poster 523 (ASM Microbe, Boston, MA, USA, 2016).

227 Spero press release: https://sperotherapeutics.com/news/press-releases/sperotherapeutics-initiates-first-clinical-study-lead-potentiatorcandidate-treatment-multidrug-resistant-gram-negativeinfections/

228 P. Brown, M. Dawson, M. Simonovic, S. Boakes and E. Duperchy, patent application WO2014/188178

229 P. Brown, M. Dawson, M. Simonovic, S. Boakes and E. Duperchy, S. J. Stanway, A. Wilson and S. F. Moss, patent application WO2015/135976

230 H. O'Dowd, B. Kim, P. Margolis, W. Wang, C. Wu, S. L. Lopez and J. Blais, Tetrahedron Lett. 2007, 48, 2003-2005.

231 P. Brown, S. Boakes, E. Duperchy, M. Simonovic, O. Abdulle, N. Divall, S. J. Stanway, A. Wilson, S. F. Moss and M. J. Dawson. Poster F-739, 55th Interscience Conference on Antimicrobial Agents and Chemotherapy (San Diego, CA, USA, 2015).

232 N. P. Wiederhold, E. Duperchy, P. Brown, L. J. Payne and M. J. Dawson Poster F-734, 55th Interscience Conference on Antimicrobial Agents and Chemotherapy (San Diego, CA, USA, 2015).

233 S. Boakes, J. H. Jorgensen, S. Boakes, M. S. Collins, M. McElmeel, M. T. Cushion, T. F. Patterson, Poster F-735, 55th Interscience Conference on Antimicrobial Agents and Chemotherapy (San Diego, CA, USA, 2015).

234 Spero press release 2017 https://sperotherapeutics.com/news/press-releases/sperotherapeutics-acquires-next-generation-antibacterialcandidates-pro-bono-bio-treatment-multidrug-resistantgram-negative-infections/

235 N. Bodor and P. Buchwald, Med. Res. Rev. 2000, 20, 58101

236 M. F. Gordeev, J. Liu, X. Wang, Z. Yuan, patent aplication WO 2016/100578

237 F. Rabanal, patent application PCT/EP2016/079066

238 D. C Hobbs, patent US 3,450,687, 1969

239 L. H. Lash, Toxicol. Appl. Pharmacol. 2005, 204, 329- 342

240 J. W. Lohr, G. R. Willsky and M. A. Acara. Pharmacol. Rev. 1998, 50, 107-142

241 L. A. Bass, M. V. Lanahan, J. R. Duncan, J. L. Erion, A. Srinivasan, M. A. Schmidt and C. J. Anderson, Bioconjugate Chem., 1998, 9, 192-200

242 H. Rudilla, E. Fusté, Y. Cajal, F. Rabanal, T. Vinuesa and M. Viñas, Molecules, 2016, 21, 1223

243 I. Karaiskos, M. Souli, I. Galani, and H. Giamarellou, Expert Opinion on Drug Metabolism \& Toxicology, 2017, 13, http://dx.doi.org/10.1080/17425255.2017.1230200 
Formatting - please delete this box prior to submission

- Graphics, including tables, will be located at the top or bottom of the column following their first citation in the text during production (unless they are equations, which appear in the flow of the text). They can be single column or double column as appropriate and require appropriate captions.

- Text is not wrapped around any of the graphics.

- During production, sufficient space will be inserted around graphics for clarity of reading; a horizontal bar will also be used to separate all inserted graphics, tables and their captions from the text:

- Please consult the Styles menu for recommended formatting for all text, including footnotes, references, tables, images and captions. 


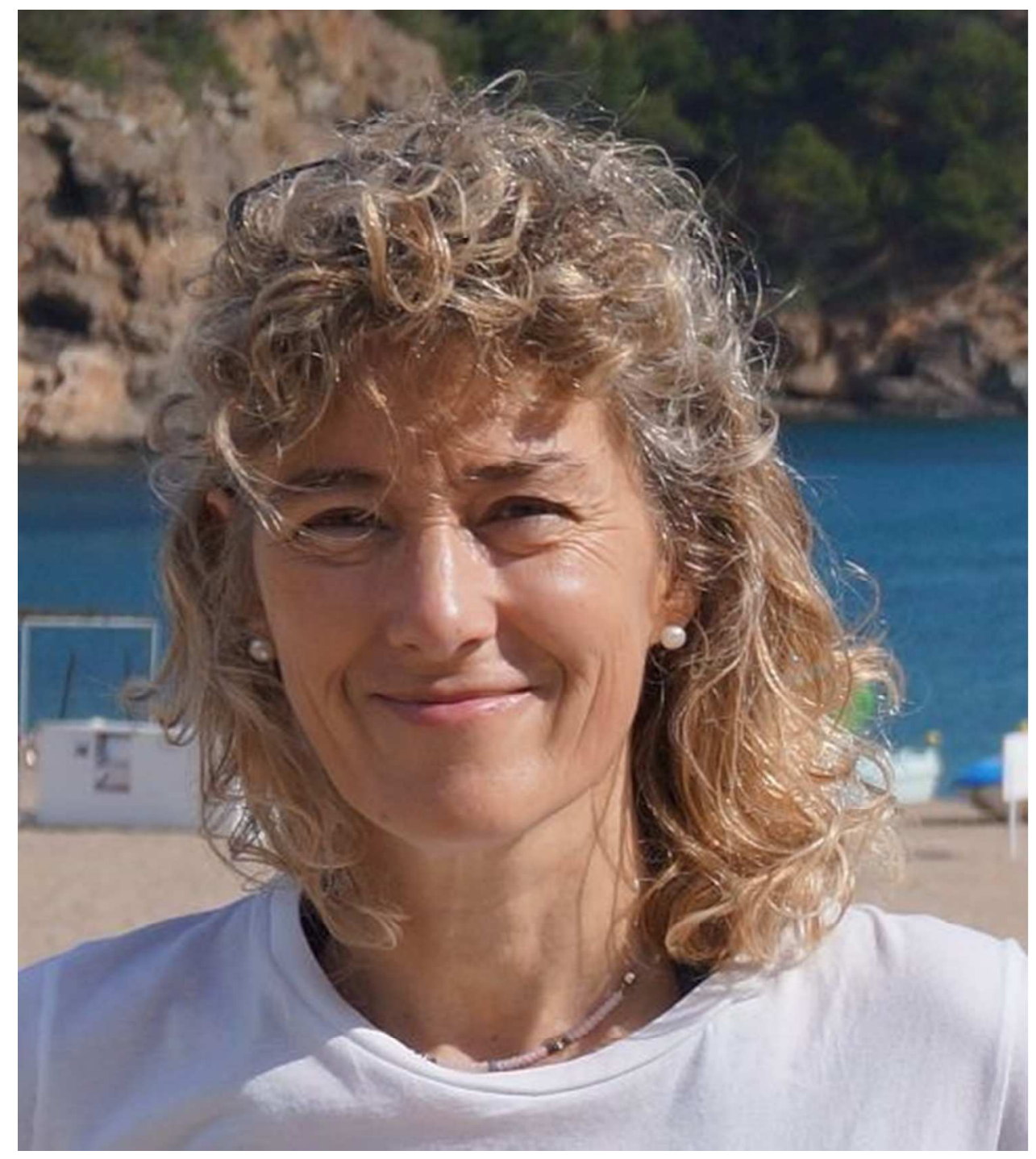

$101 \times 114 \mathrm{~mm}(120 \times 120 \mathrm{DPI})$ 


\section{Natural Products Reports}

\section{Recent advances and perspectives in the design and development of polymyxins}

Received 00th January 20xx, Accepted 00th January 20xx

DOI: $10.1039 / \times 0 \times x 00000 x$

www.rsc.org/

\author{
Francesc Rabanal $^{\mathrm{a}^{*}}$ and Yolanda Cajal ${ }^{\mathrm{b}}$ \\ Covering: 1947-early 2017, particularly from 2005-early 2017
}

The rise of bacterial pathogens with acquired resistance to almost all available antibiotics is becoming a serious public health issue. Polymyxins, antibiotics that were mostly abandoned a few decades ago because of toxicity concerns, are ultimately considered as a last-line therapy to treat infections caused by multi-drug resistant Gram-negative bacteria. This review surveys the progress in understanding polymyxin structure, chemistry, mechanisms of antibacterial activity and nephrotoxicity, biomarkers, synergy and combination with other antimicrobial agents and antibiofilm properties. An update of recent efforts in the design and development of a new generation of polymyxin drugs is also discussed. A novel approach considering the modification of the scaffold of polymyxins to integrate metabolism and detoxification issues into the drug design process is a promising new line to potentially prevent accumulation in kidney and reduce nephrotoxicity.

\section{Introduction \\ 2. Naturally ocurring polymyxins \\ 3. Antimicrobial profile \\ 4. Commercially available polymyxins \\ 5. Mechanism of action \\ 6. Resistance to polymyxins \\ 7. Combination with other antibiotics \\ 8. Synergy with antifungals \\ 9. Antibiofilm activity \\ 10. Toxicity \\ 10.1. Mechanism of nephrotoxicity \\ 10.2 Biomarkers of nephrotoxicity}

11. Design and development of new polymyxins

11.1 Synthetic preparation of polymyxin analogs

11.2 CB-182,804 analog

11.3 Pfizer $5 x$ analog

11.4 Monash FADDI analogs

11.5 Queensland analogs

11.6 Northern Antibiotics analogs

11.7 Cantab analogs

11.8 Scaffold modified polymyxins

11.9 MicuRx analogs

11.10 Barcelona analogs

12. Conclusions and perspectives

13. Acknowledgements

14. References

\footnotetext{
a. Organic Chemistry Section, Department of Inorganic and Organic Chemistry, Faculty of Chemistry, University of Barcelona. E-mail: frabanal@ub.edu ${ }^{b}$ Department of Pharmacy, Pharmaceutical Technology and Physical Chemistry, Faculty of Pharmacy and Food Sciences, University of Barcelona. E-mail: ycajal@ub.edu ${ }^{b}$ Institute of Nanoscience and Nanotechnology (IN2UB), University of Barcelona. + Footnotes relating to the title and/or authors should appear here.

Electronic
}

\section{Introduction}

Polymyxins are a group of antimicrobial cyclic lipopeptides discovered in 1947.1-3 They are produced by fermentation of strains of Paenibacillus polymyxa (formerly known as Bacillus polymyxa). Polymyxins consist of a heterogeneous mixture composed of up to 30 closely related lipopeptides (Table I). ${ }^{4-7}$ The term "polymyxin" is accepted as the general name for this class of antibiotics produced by P. polymyxa. Polymyxin B and colistin (polymyxin E) are the most known members of this family as they were commonly used as antibiotics in hospitals from late 1950s to late 1970s, approximately. Then, they were gradually withdrawn from the clinical practice due to toxicity issues such as adverse neurological effects and most importantly, nephrotoxicity concerns. In addition, novel aminoglycosides (gentamicin) and second- and third-generation cephalosporins showing less toxic side effects became available. ${ }^{8}$ However, the emergence of Gram-negative bacteria that are resistant to almost all classes of available antibiotics has resulted in the rescue of polymyxins as a last resort for patients whose other treatment options were limited.

Antibiotic resistance is becoming a serious public health issue. In the USA, for instance, at least 2 million people are infected by antibiotic-resistant bacteria and at least 23,000 people die each year as a direct consequence. ${ }^{9,10}$ A similar situation is taking place in Europe. ${ }^{11,12}$ The WHO has recently issued a list of the most critical pathogenic bacteria for which new antibiotics are urgently needed: carbapenem-resistant strains of Acinetobacter baumannii, Pseudomonas aeruginosa, and Enterobacteriaceae. ${ }^{13}$

Polymyxin B and colistin are now used as a last-line therapy to treat infections caused by multi-drug resistant bacteria such as $P$. aeruginosa, A. baumannii, Klebsiella pneumoniae, and Escherichia coli . These bacteria are part of the so-called ESKAPE bacteria, thus nicknamed by the Infectious Disease Society of America that 
Table 1: Structure of naturally occurring polymyxins B and E (colistin), clinically relevant members of the polymyxin family§

\begin{tabular}{|l|l|l|l|}
\hline Polymyxin & Fatty acyl tail & Aa 6 & Aa7 \\
\hline B1 & (S)-6-methyloctanoyl & D-Phe & Leu \\
\hline B1-Ile & (S)-6-methyloctanoyl & D-Phe & Ile \\
\hline B2 & 6-methyheptanoyl & D-Phe & Leu \\
\hline B3 & octanoyl & D-Phe & Leu \\
\hline B4 & heptanoyl & D-Phe & Leu \\
\hline B5 & nonanoyl & D-Phe & Leu \\
\hline B6 & 3-hydroxy-6-methyloctanoyl & D-Phe & Leu \\
\hline E1 & (S)-6-methyloctanoyl & D-Leu & Leu \\
\hline E2 & 6-methyheptanoyl & D-Leu & Leu \\
\hline E3 & octanoyl & D-Leu & Leu \\
\hline E4 & heptanoyl & D-Leu & Leu \\
\hline E7 & 7-methyloctanoyl & D-Leu & Leu \\
\hline E1-Ile & (S)-6-methyloctanoyl & D-Leu & Ile \\
(circulin A) & & & \\
\hline E1-Val & (S)-6-methyloctanoyl & D-Leu & Val \\
\hline E1-Nva & (S)-6-methyloctanoyl & D-Leu & Nva \\
\hline E2-Val & 6-methyheptanoyl & D-Leu & Val \\
\hline E2-Ile & 6-methyheptanoyl & D-Leu & Ile \\
\hline E8-Ile & 7-methylnonanoyl & D-Leu & Ile \\
\hline
\end{tabular}

Table 2: Structure of additional naturally occurring polymyxins§

\begin{tabular}{|l|l|l|l|l|l|}
\hline Polymyxin & Fatty acyl tail & Aa3 & Aa6 & Aa7 & Aa10 \\
\hline A1 & 6-methyloctanoyl & D-Dab & D-Leu & Thr & Thr \\
\hline A2 & 6-methylheptanoyl & D-Dab & D-Leu & Thr & Thr \\
\hline C1 & 6-methyloctanoyl & Dab & Phe & Thr & Thr \\
\hline C2 & 6-methylheptanoyl & Dab & Phe & Thr & Thr \\
\hline D1 & 6-methyloctanoyl & D-Ser & D-Leu & Thr & Thr \\
\hline D2 & 6-methylheptanoyl & D-Ser & D-Leu & Thr & Thr \\
\hline F1 & 6-methyloctanoyl & \multicolumn{2}{|c|}{ (Dab x5, Thr, Leu x2, Ser, Ile) } \\
\hline F2 & 6-methylheptanoyl & \multicolumn{2}{|c|}{ (Dab x5, Thr, Leu x2, Ser, Ile) } \\
\hline M1 & 6-methyloctanoyl & Dab & D-Leu & Thr & Thr \\
\hline M2 & 6-methylheptanoyl & Dab & D-Leu & Thr & Thr \\
\hline S1 & 6-methyloctanoyl & D-Ser & D-Phe & Thr & Thr \\
\hline T1 & 6-methyloctanoyl & Dab & D-Phe & Leu & Leu \\
\hline T2 & 6-methylheptanoyl & Dab & D-Phe & Leu & Leu \\
\hline PMB1 & 6-methyloctanoyl & D-Dab & D-Phe & Thr & Thr \\
\hline PMB2 & 6-methylheptanoyl & D-Dab & D-Phe & Thr & Thr \\
\hline P1 & 6-methyloctanoyl & D-Dab & D-Phe & Thr & Thr \\
\hline P2 & 6-methylheptanoyl & D-Dab & D-Phe & Thr & Thr \\
\hline
\end{tabular}

$\S$ The reader may find nomenclatures such as "iso" and "ante-iso" fatty acids in the literature. For instance, isooctanoic acid, $\mathrm{i}-\mathrm{C}_{8}$ corresponds to 6-methylheptanoic acid. Anteisononanoic acid, $a-C_{9}$, corresponds to 6 methyloctanoic acid and may appear in two different configurations, $R$ or $S$, since a stereocenter is present in carbon 6 . The configuration of this stereocenter is expected to be $S$ as the branch-chain fatty acid synthesizing system starts in the case of anteiso-fatty acids from the $\alpha$-ketoacid of isoleucine as primer. Isoleucine has an $S$ configuration in carbon 3 , in its sidechain.

has proposed to pursue a global commitment to develop 10 new antibacterial drugs by 2020 (the 10 x '20 Initiative). "ESKAPE" stands for the initials of the above mentioned Gram-negative bacteria together with Gram-positive Enterococcus faecium and Staphylococcus aureus. ${ }^{9}$

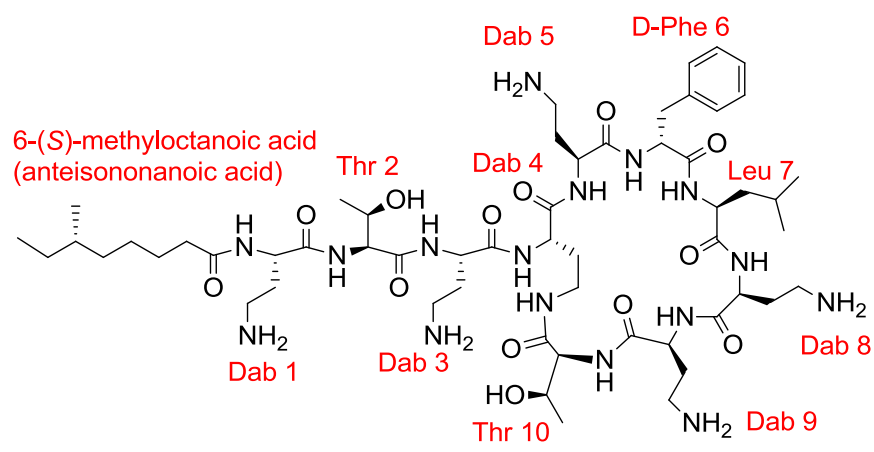

Figure 1: Polymyxin B1 as an example of the general structure of polymyxins. Amino acid positions are numbered from 1 to 10 .

The objective of this review is to gather and analyze the background in the field of polymyxins, highlighting the efforts and new approaches carried out by different groups worldwide toward the design and development of new polymyxin-based compounds potentially capable of overcoming the current drawbacks of the natural compound, particularly, nephrotoxicity. Other fundamental reviews in the field of polymyxins have been published in the last years. As the present manuscript will mainly but not only concentrate in the last decade achievements and new approaches, the reader is also addressed to reviews by Vaara, Velkov\&Li, Brown\&Dawson for a previous background in the area. ${ }^{14-17}$

\section{Naturally occuring polymyxins}

The Paenibacillus genus (previously included in the genus Bacillus) comprises tenths of species that are facultative anaerobic and endospore-forming bacteria. In particular, strains of Paenibacillus polymyxa thrive in the plant rizhosphere, are capable of fixing nitrogen, suppress some plant diseases and promote a healthy growth in plants, such as crops and trees. Hence, $P$. polymyxa strains are used as an effective alternative to the chemical control against a wide set of plant pathogenic fungi and bacteria. Polymyxins, including colistin and circulin, are the main class of peptide antibiotics produced by most strains of $P$. polymyxa although other compounds are produced as well. Other strains produce peptides such as polyxins, polypeptins or fuaricidins. ${ }^{18}$

The general structure of polymyxins consists of a cyclic heptapeptide unit (amino acids 4-10) and a lipotripeptide that bifurcates from the fourth amino acid of the sequence (Figure 1). The lipid unit capping the N-terminal amino acid is a linear or branched fatty acyl moiety, that together with amino acids in the 6th and 7th position define the hydrophobic features of the molecule. The rest of amino acid residues are polar (L-threonines) and amino-containing basic residues (2,4-diaminobutanoic acid) that provide polymyxins with its polycationic nature at physiological $\mathrm{pH}$. Polymyxins are secondary metabolites generated by non-ribosomal peptide synthetase enzyme complexes. Hence, they contain non-proteinogenic amino acids as well (not present in regular coded proteins) such as the above mentioned 2,4-diaminobutanoic acid, D-phenylalanine or D-Leucine. 
The first polymyxins discovered were reported almost simultaneously by three different teams in 1947: Benedict and Langlykke, ${ }^{1}$, Stansly and coworkers ${ }^{2}$ and Ainsworth and coworkers. ${ }^{3}$ Ainsworth called the antibiotic "aerosporin" since it was obtained from Bacillus aerosporus which later was found to be B. polymyxa, essentially the same studied by Benedict and Langlykke. The antimicrobial "aerosporin" is now known as polymyxin A, whereas the so-called "polymyxin" described by Stansly corresponds to polymyxin D. Further investigations by Brownlee and Bushby managed to isolate a third type of polymyxin, which was named polymyxin $B$, and later, polymyxins $C$ and $E$ were found. Polymyxins A, B, C, D and E showed similar antibacterial activity, but an in vivo assay of nephrotoxicity in Wistar rats by estimating the total protein excreted in the urine indicated a striking increase of proteinuria for polymyxins $A, C$ and $D$ but not for polymyxins $B$ and $E .{ }^{19}$

In parallel, Koyama described in 1950 the isolation of an antibiotic from a culture broth of a new strain they named Bacillus polymyxa var. colistinus (Aerosporus colistinus). ${ }^{20}$ The new compound was called colistin, and chemical determination proved it to be cyclic and share an amino acid composition qualitatively identical to that of polymyxin E described by Brownlee. ${ }^{21}$

In 1948, Tetrault and coworkers isolated another antibiotic peptide that named circulin as it was produced by Bacillus circulans. It was found to share a similar composition to the one of polymyxins known so far. It was also active against Gram-negative bacteria. In the following years, other members of the family were discovered, such as polymyxin $\mathrm{M}$ found in the soil of Moscow by Khokhlov and coworkers. $^{21,22}$ More recently, Martin and coworkers have isolated mattacin, a cyclic lipodecapeptide produced by Paenibacillus kobensis $\mathrm{M}$ and found by structure elucidation to be identical to polymyxin $\mathrm{M}^{23}$

Polymyxin $\mathrm{P}$, described for the first time in 1969 , has been recently found to be the main compound produced by $P$. polymyxa M-1. Polymyxin $\mathrm{P}$ suppressed the growth of phytopathogenic Enterobacteriaceae bacteria Erwinia amylovora Ea 273, and E. carotovora, the causative agents of fire blight (in apples and pears) and soft rot, respectively. Hence, it has been proposed as an alternative of chemical bactericides to control these and other plant diseases caused by Gram-negative bacteria. ${ }^{24,25}$

Other polymyxins, such as polymyxin S1 and T1 were isolated from P. polymyxa Rs-6 and E-12, respectively. Polymyxin T1 was found to be active not only against Gram-negative bacteria but also against Gram-positive bacteria, a characteristic shared with polymyxin M. ${ }^{25-29}$ Polymyxin F, produced by Bacillus circulans ATCC 31228 , has also been described. ${ }^{30}$ Similarly, polymyxin $C$ has also been reported (see above) but apparently, has not been subjected to detailed structural studies. ${ }^{28,31,32}$

The last members of the polymyxin family described so far were diasteromers of polymyxin B, named PMB1 and PMB2. ${ }^{33}$ They were produced by $P$. polymyxa PKB-1 and had a D-Dab amino acid in position 3 rather than the usual L-Dab. Their sequences were elucidated by high-resolution mass spectrometry, MS/MS sequencing, and the stereochemistry, by chiral gas chromatography.

Finally, it is worth mentioning a family of natural products called octapeptins, first reported in the mid 1970's. ${ }^{22}$ They are closely related to polymyxins as both families share a similar structure. They consist of a cyclic polycationic peptide sequence, containing a high percentage of 2,4-diaminobutanoic acid and a fatty acyl tail bound through an amide linkage. However, octapeptins contain eight amino acid residues as it may be deduced from its name, with a single exocyclic amino acid stemming from the cycloheptapeptide moiety. A review on octapeptins has recently been published in this journal so the reader is kindly referred to this document for further information. ${ }^{34}$

\section{Antimicrobial profile}

Polymyxins are narrow-spectrum antibiotics since they are only active against Gram-negative bacteria including multi-drug resistant (MDR) strains. This includes some non-fermenting bacteria such as $P$. aeruginosa and Acinetobacter spp. and some members of the Enterobacteriaceae family, Escherichia spp, Klebsiella spp, Enterobacter spp, Citrobacter spp, Salmonella spp, Shigella spp and Haemophilus spp, and Pasteurella spp. However, Proteus spp, Burkholderia spp, Serratia spp (i. e. Serratia marcescens) and Moraxella spp (i. e. Moraxella catarrhalis) and genera Brucella, Neisseria, Chromobacterium and Providencia, have intrinsic resistance to polymyxins. ${ }^{35}$ Finally, it has been reported that polymyxin $E$ was found to be active against some mycobacterial species, e.g. Mycobacterium xenopi, $M$. intracellulare, $M$. tuberculosis, $M$. fortuitum, $M$. phlei and $M$. smegmatis.36,37 Polymyxin B also exhibited activity against Cryptococcus neoformans fungus. ${ }^{38}$

\section{Commercially available polymyxins}

Since polymyxins are manufactured by fermentation procedures, they have a heterogeneous composition. They contain several structurally related components such as isomers and homologous compounds (Table 1). For instance, the major constituents of polymyxin B obtained from $P$. polymyxa are the related polymyxins B1, B2, B3 and B1-lle, differing only in the fatty acyl moiety and the amino acid in position 7 (Leu or lle). Their composition and antibacterial activity have been recently studied in detail. A typical proportion of components in polymyxin B would be $c a 70-74 \%$ of the B1 type, $13-16 \%$ of B2, 3-5\% of B3 and around 8-9 \% of Ile-B1. ${ }^{39,40}$ The activity of some of these components have been assessed individually in strains of $P$. aeruginosa, $A$. baumannii and $K$. pneumoniae including multi-drug resistant isolates. The results of antimicrobial activity measured by means of their MIC (minimal inhibitory concentration) showed differences within the variability generally accepted for such a kind of assays. Apparently, the minor structural differences (length of the fatty acid tail and compound isomers) among the components did not affect much their in vitro potency. ${ }^{40,41}$ Nevertheless, it is worth mentioning that one of the minor components, polymyxin B3, showed higher activity than the rest against $P$. aeruginosa, $E$. coli and $K$. pneumoniae 
while the compound B1-lle was more potent against $A$. baumannii. In the same study, synergism between the different members of the polymyxin B mixture following checkerboard analyses was explored. The tests revealed that the combination of polymyxins B3 and B1-lle met the criteria for synergy against Enterobacteriaceae whereas the major components polymyxin B1 and B2 showed a low probability of synergy when combined. ${ }^{42}$

Regarding the pharmacokinetics of individual polymyxin B major components, no considerable differences were detected among them when tested in the rat animal model and in humans. In fact, it has been proposed as a reasonable approach to use the combined concentration values of the individual polymyxin components in pharmacokinetic studies to estimate overall drug exposure to polymyxin B. ${ }^{43}$

In another study comparing polymyxin B and colistin in the rat model, the individual major components of polymyxin $B$ (B1 and $B 2$ ) and colistin ( $A$ and $B$ ) yielded similar pharmacokinetic parameters such as clearance, volume of distribution, elimination half-life, and urinary recovery. Notwithstanding that, colistin A (polymyxin E1) and colistin $B$ (polymyxin E2) displayed lower protein binding in rat plasma compared to polymyxins B1 and B2.

In relation with the accumulation of the individual products (B1, B1Ile, B2+B3) in kidney, a fact that correlates with nephrotoxicity (see section 10 below), the relative proportions of the components present in the renal tissue at $48 \mathrm{~h}$ were found to be comparable to the concentrations in the USP (United States Pharmacopeia) mixture indicating no preferential accumulation of any of the components. ${ }^{44,45}$

Commercial polymyxin B is available in the sulfate form either for parenteral (intravenous and intramuscular), topical (ophthalmic and otic instillation), and intrathecal use (in cases of MDR Gram-negative caused meningitis). The dosage of intravenous polymyxin $B$ is generally $1.5-2.5 \mathrm{mg} / \mathrm{kg} /$ day $(15.000-25.000 \mathrm{IU} / \mathrm{kg} / \mathrm{day} ; 1 \mathrm{mg}$ of polymyxin B corresponds to $c a 10.000 \mathrm{IU}$, International Unit), However, commercial formulations of polymyxin $B$ are not always available in many countries of the world. Injectable polymyxin $B$ and colistin formulations are available only in Brazil, Malaysia, Singapore and the USA while in Europe and Australia, colistimethate is the only parenteral formulation that can be found. For the treatment of eye infections caused by $P$. aeruginosa, $0.1-0.25 \%$ polymyxin $B$ solutions (10.000 IU to $25.000 \mathrm{IU} / \mathrm{ml}$ ) are recommended. Polymyxin B in combination with a local anaesthetic (i. e. lidocaine, procaine) can also be found for intramuscular administration, in eardrops, and ointments. Combination with hydrocortisone is also available for otic use. ${ }^{15,46}$

Colistin has two commercially available forms: colistin sulfate and sodium colistimethate (sodium colistin methanesulfonate, CMS, Figure 2). ${ }^{47}$ Both contain different proportions of colistin A (polymyxin E1), and colistin B (polymyxin E2), which account for more than $80 \%$ of colistin, together with many other minor components. ${ }^{6}$ Colistin sulfate may be administered orally for bowel decontamination or topically as a powder for the treatment of
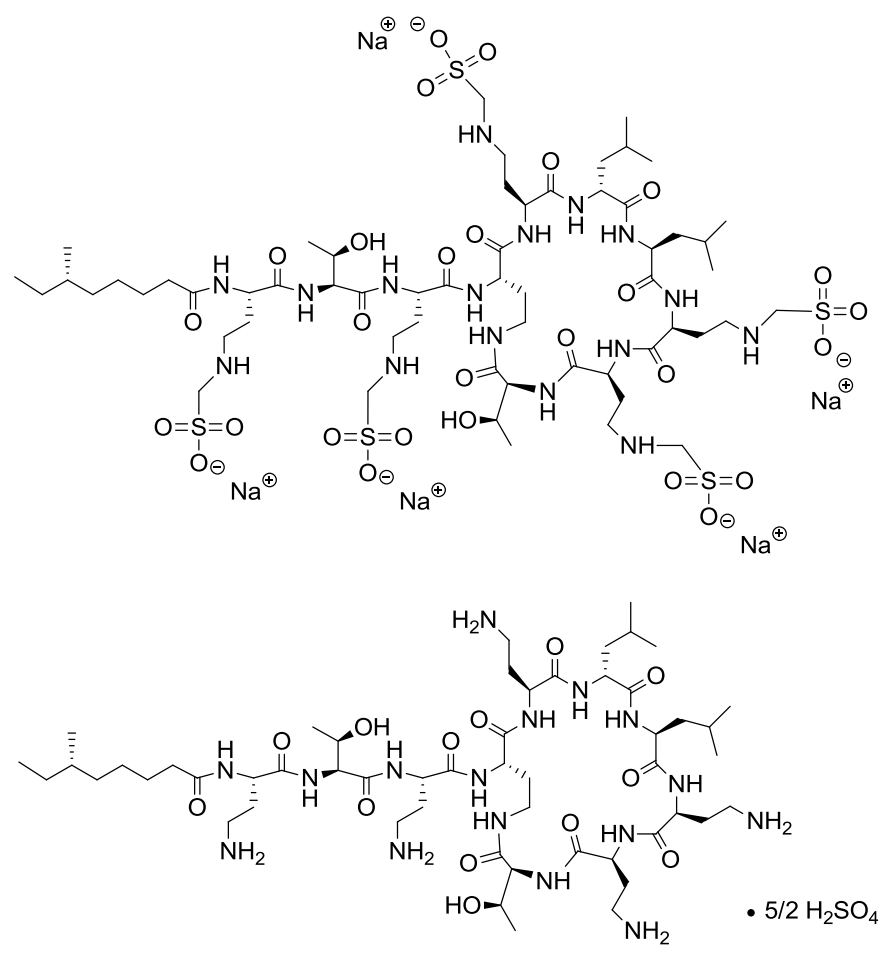

Figure 2: Structure of sodium colistimethate and colistin A (polymyxin E1). Molecular weight of colistimethate is $1749.81 \mathrm{~g} \cdot \mathrm{mol}^{-1}$ $\left(\mathrm{C}_{58} \mathrm{H}_{105} \mathrm{~N}_{16} \mathrm{O}_{28} \mathrm{~S}_{5} \mathrm{Na}_{5}\right.$ ) while for the free base (devoid of sodium methylsulfonate derivatisation) it is $1169.48 \mathrm{~g} \cdot \mathrm{mol}^{-1}\left(\mathrm{C}_{53} \mathrm{H}_{100} \mathrm{~N}_{16} \mathrm{O}_{13}\right)$. Hence, $1 \mathrm{mg}$ of colistimethate corresponds to $0.67 \mathrm{mg}$ of free base colistin (or $0.81 \mathrm{mg}$ of colistin sulfate, assuming 2.5 mols of sulfate per mol of colistin)

bacterial skin infections. Colistimethate is a prodrug of colistin. It is produced by the reaction of colistin with formaldehyde and sodium hydrogensulfite (see section 10 below). It is less toxic than colistin sulfate since it is polyanionic, but devoid of antimicrobial activity. It can be administered parenterally (intravenously, intramuscularly), intrathecally, intraventricularly or by inhalation (aerosolized, to treat respiratory tract infections caused by multidrug-resistant Gramnegative bacteria, and cystic fibrosis). Colistimethate as a prodrug, slowly reverts to colistin although hydrolysis is not always complete. ${ }^{48,49}$ It has been reported that only a $31.2 \%$ of $\mathrm{CMS}$ is hydrolysed in vitro to colistin in 4 hours at $37^{\circ} \mathrm{C}$ in human plasma..$^{50}$ In vivo, even smaller proportions of colistimethate are converted to colistin. ${ }^{51}$ This has been attributed to the slow hydrolysis rate of colistimethate to colistin combined with a fastest rate of renal clearance. In fact, it is estimated that only ca $25 \%$ of colistimethate is converted to active colistin in patients with normal renal function. ${ }^{52}$

Colistimethate intravenous dosage in adult patients with normal renal function are different in Europe and USA. In Europe colistimethate doses are in general 4-6mg/kg (50.000-75.000 IU/kg daily; $1 \mathrm{mg}$ of colistimethate corresponds to $12.500 \mathrm{IU})$. In France and Austria, it reaches values of $12 \mathrm{mg} / \mathrm{kg}$ ( $150000 \mathrm{IU} / \mathrm{kg} / \mathrm{day})$. In the USA, the recommended dose is $2.5-5 \mathrm{mg} / \mathrm{kg}$ expressed in terms of colistin base, equivalent to $6-12 \mathrm{mg} / \mathrm{kg}$ of colistimethate, (75000-150000 $\mathrm{IU} / \mathrm{kg})^{46,54}$ 
Examples of polymyxin drug brands approved by the FDA and present in the market include Pediotic ${ }^{\circledR}$ (neomycin, polymyxin B sulfate and hydrocortisone suspension, USP, for otic use), Polysporin ${ }^{\circledR}$ (polymyxin B, zinc bacitracin and gramicidin; aerosol, topical, and ophthalmic), Neosporin ${ }^{\circledR}$ (polymyxin B, zinc bacitracin and neomycin triple ointment) and Polytrim ${ }^{\circledR}$ (polymyxin B sulfate and trimethoprim ophthalmic solution, USP). Colomycin ${ }^{\circledR}$ and Coly-Mycin ${ }^{\circledR}$ contain colistin methanesulfonate as the active principle (1-2 million units, for injection). Polymycin B sulfate (polymyxin B sulfate) was approved by the FDA in 2011 for the treatment of infections caused by resistant strains of $P$. aeruginosa, H. influenzae, E. coli, Enterobacter aerogenes, and K. pneumoniae by parenteral administration. ${ }^{32}$

\section{Mechanism of action}

Polymyxins have a narrow antimicrobial spectrum with selectivity for Gram-negative bacteria. This is because the first molecular target of these polycationic lipopeptides is the lipopolysaccharide (LPS), the main component of the outer membrane (OM) of Gram-negatives. The low permeability of the two-membrane cell envelope in Gramnegative bacteria is the main reason for the low hit rate in the discovery of new antibiotics against this bacterial class..$^{55}$ The outer membrane is an asymmetric bilayer of LPS in the outer monolayer, and a mixture of phospholipids in the inner. ${ }^{56,57}$ LPS is composed of three domains: lipid A, central core oligosaccharide, and outermost O-antigen chain. ${ }^{58}$ Lipid $A$ is the domain responsible for outer membrane thigh packing, and the principal target for polymyxins. It contains an $\mathrm{N}$ - and O-acylated diglucosamine bisphosphate backbone, and forms a highly packed structure. Although there are many variations among species, in polymyxin-sensitive bacteria LPS has several anionic charges, responsible for the strong electrostatic interactions with polycationic polymyxins. LPS molecules are bridged and partly neutralized by divalent ions $\mathrm{Ca}^{2+}$ and $\mathrm{Mg}^{2+}$, thus conferring a high rigidity and low permeability to the outer membrane. ${ }^{56,59}$

The antimicrobial activity of polymyxins begins by competitive displacement of membrane-stabilizing divalent cations $\mathrm{Ca}^{2+}$ and $\mathrm{Mg}^{2+}$, thus causing a destabilization of the LPS layer and allowing insertion of the hydrophobic acyl chain of the antibiotic, which locates in the hydrophobic domain of lipid A. ${ }^{60,61}$ This causes an expansion of the LPS monolayer ${ }^{15,62}$ and results in disruption of the outer membrane permeability barrier, facilitating the entrance of polymyxin into the periplasmic space, a process of self-promoted uptake first described by Hancock. ${ }^{63,64}$ Binding to LPS is a required first step for antibacterial activity, but it is not enough. For example, deacylated polymyxin $\mathrm{B}$ nonapeptide, lacking the $\mathrm{N}$-terminal acyl chain and Dab ${ }^{1}$ residue, is an extremely poor antibiotic, but is still capable of binding to LPS and preserving a significant OMpermeabilizing action. ${ }^{14}$ This susceptibility explains the drastic sensitizing action of the nonapeptide, allowing other small molecules (such as conventional antibiotics) to cross the outer membrane. 65 In addition, polymyxin resistance is related to lipid A modification with phosphoethanolamine and/or galactosamine, or to the complete loss of LPS, thus avoiding binding of polymyxins to the OM. ${ }^{66}$
The interaction of PxB and PXB nonapeptide with LPS has been studied in detail at the molecular level, and involves hydrophobic as well as electrostatic interactions. The structure of PxB bound to LPS has been determined by NMR spectroscopy, ${ }^{67-70}$ and consists in an envelope-like fold of the peptide ring separating the polar/charged residues from the hydrophobic components, conferring an amphiphilic character to the structure. It is postulated that the $\beta$-turn structure is stabilized by hydrophobic interactions involving two hydrophobic domains on the lipopeptide DPhe ${ }^{6}-$ L-Leu $^{7}$ domain on the peptide ring, and the $\mathrm{N}$-terminal fatty acid chain, with the aliphatic chains of lipid A. ${ }^{65,68}$ The electrostatic interactions between the positive side chains of Dab residues on PxB and two of the negative phosphate groups of the phosphorylated lipid $A$ headgroups are essential for complex formation, whereas the hydrophobic interactions are responsible for insertion into the outer membrane hydrophobic core. The structure of PxB nonapeptide bound to LPS has been determined by tranferred nuclear Overhauser effect NMR and molecular dynamics, ${ }^{70}$ and is consistent with surface binding of the peptide, without insertion into the hydrophobic core of lipid A. This will explain the lack of antibiotic activity, since the nonapeptide will not reach the inner membrane.

Once polymyxin has crossed the outer membrane, it must interact with the cytoplasmic or inner membrane in order to kill the bacteria. ${ }^{11}$ The inner membrane in Gram-negative bacteria is mostly composed of zwitterionic phospholipid phosphatidylethanolamine (PE) and anionic phosphatidylglycerol (PG) and cardiolipin (CL). All bacteria have at least a $15 \%$ of anionic lipids, but this can be either $\mathrm{PG}$ or $\mathrm{CL}$ or both. ${ }^{57}$ The same lipids are found in the inner layer of the outer membrane, although the proportions are different. ${ }^{71}$ The mechanism of bacterial killing is not related with membrane permeation, which takes place at concentrations well above the minimal inhibitory concentration. ${ }^{62,72,73} \mathrm{~A}$ threshold concentration of PXB is required on the membrane to form clusters that insert and form depolarizing ion-permeable pores, however dissipation of the $\mathrm{pH}$ gradient is not observed in E. coli after PxB treatment, and the bactericidal effect is expressed at lower concentrations and is not dependent on depolarization of the outer membrane. ${ }^{74}$ Although a mechanism of bacterial killing based on disruption of the physical integrity of the cytoplasmic membrane by pore formation or even a detergent effect are demonstrated for other $\mathrm{AMPs}^{75}$ in the polymyxin family such effects only occur at high peptide/lipid ratios.

A more likely mechanism of action has been described for polymyxin $B$ that involves contact formation between the outer and inner membranes of Gram-negative bacteria, ${ }^{71,76}$ also seen in other antimicrobial peptides such as cecropins, ${ }^{77}$ and $\mathrm{rBPI}_{21} .{ }^{78}$ According to this model, once in the periplasmic space stoichiometric amounts of polymyxin will form contacts between the two enclosed phospholipid interfaces, and promote a fast and selective exchange of anionic phospholipids. The resulting changes in the membrane lipid composition trigger an osmotic imbalance that leads to bacterial stasis and cell death. ${ }^{79}$ Biophysical studies using model membranes have demonstrated that at the concentrations around the MIC, PxB and colistin induce the apposition of anionic vesicles with a composition that mimics the bacterial membrane, and the formation of functional vesicle-vesicle contacts. ${ }^{80}$ These contacts support a fast 
and selective exchange of phospholipids exclusively between the outer monolayers of the vesicles in contact and maintaining intact the inner monolayers and the aqueous contents. ${ }^{81}$ For example, monoanionic phospholipids such as phosphatidylglicerol are transferred through the contacts, whereas zwitterionic phosphatidylcholine or dianionic phosphatidic acid are excluded, independently of the composition of the fatty acid chains. The nonantibiotic derivative polymyxin $B$ nonapeptide is not able to induce vesicle-vesicle contacts. ${ }^{82}$ Sublethal concentrations of PxB in growing $E$. coli induce a highly selective cellular stress, with transcription of the osm $Y$ gene without leakage of solutes and protons. $71,77,79$ Since osm $Y$ expression is also induced by hyperosmotic stress, encoding a periplasmic protein that protects from cell membrane damage, ${ }^{83}$ the interpretation is that PxB forms functional contacts in the periplasmic space between the anionic phospholipid-containing outer surface of the cytoplasmic membrane and the inner surface of the outer membrane. ${ }^{84}$ The consequent loss of phospholipid compositional specificity caused by the PXBmediated exchange can be the origin of the osmotic imbalance that leads to bacteriostasis and cell death. ${ }^{14}$ An analysis of the transcriptome of $A$. baumannii exposed to colistin shows that this antibiotic alters the expression of a very large number of genes, many of them involved in the synthesis and transport of membrane components. This is consistent with the inner membrane-outer membrane lipid exchange mechanism of action with alteration of the normal membrane composition. ${ }^{85}$

The structure of polymyxin in the IM-OM contacts has been characterized in lipid vesicles by fluorescence resonance energy transfer, using labelled derivatives of a synthetic PxB analog, sp-B. ${ }^{86}$ Intermolecular FRET is consistent with self-association of the peptide, possibly forming dimers, when bound to anionic vesicles at concentrations that are relevant for formation of vesicle-vesicle contacts and lipid exchange. Atomic force microscopy of polymyxin bound to monolayers of $E$. coli lipid extract shows structures that are consistent with the formation of aggregates of several particles at the concentrations that induce contact formation. ${ }^{82}$

A series of polymyxin analogs obtained by solid phase synthesis and including a disulfide bond gave additional information on the mechanism of action. For example, conservative analogs maintaining the main structural characteristics of polymyxin, namely 5 positive charged residues, a cyclic heptapeptide, a lineal tripeptide and the $\mathrm{N}$-terminal acyl chain, are also active against Gram-negative bacteria and induce vesicle-vesicle contacts and a selective lipid exchange similar to polymyxin. ${ }^{87,88}$ However, substitution of Dab residues for Arg results in more lytic lipopeptides, with a different spectrum of activity that includes Gram-positive and Gram-negative bacteria. ${ }^{89,90}$ Flow citometry of $E$. coli treated with one of the Arg-containig polymyxin analogs shows that depolarization and permeabilization take place roughly at the same time, and are consistent with a membrane-based mechanism of action. ${ }^{89,91}$ It has also been shown that polymyxin $\mathrm{B}$ and colistin are able to inhibit the vital respiratory enzyme type II NADH-quinone oxidoreductase (NDH-2) in the inner membrane of Gram-negative bacteria, although $\mathrm{IC}_{50}$ values are high. ${ }^{92}$

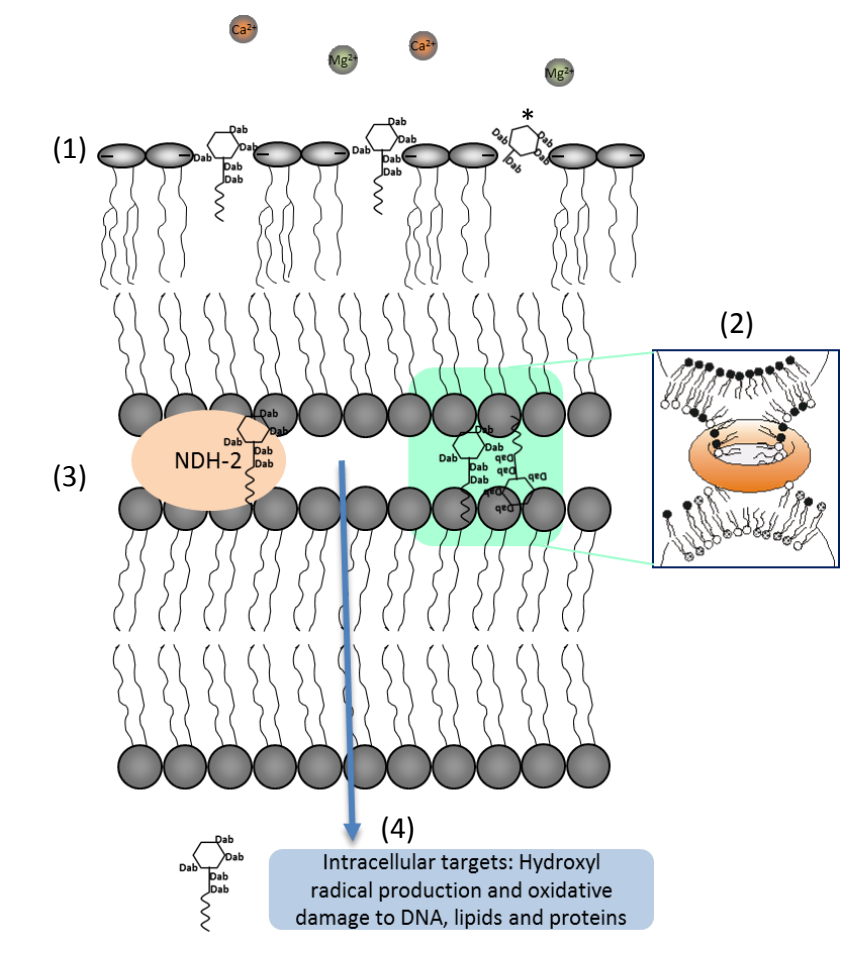

Figure 3: Representation of the putative mechanism of action of polymyxin on Gram-negative bacteria. (1) Displacement of $\mathrm{Ca}^{2+}$ and $\mathrm{Mg}^{2+}$ and binding to lipid $A$; (2) Self-promoted uptake to the periplasmic space and formation of OM-IM contacts and lipid exchange; (3) Inhibition of respiratory enzyme type II NADH-quinone oxidoreductase; (4) Entry into the cytoplasm and access intracellular targets. *Polymyxin B nonapeptide activity is limited to step (1).

Interestingly, polymyxin B nonapeptide has no inhibitory activity, in agreement with its reported inability to cross the cell membrane. NADH-2 inhibition has also been identified in other compounds including phenothiazines, quinolinyl pyrimidines and quinolones, but in all cases the mode of action remain unclear and in the case of polymyxins is considered a secondary mechanism of action..$^{93}$ Polymyxin $B$ and $E$ can inhibit alternative membrane bound respiratory enzymes nicotinamide adenine dinucleotide dehydrogenase and malate:quinone oxidoreductase in Mycobacterioum smegmatis. ${ }^{36}$

Some reports suggest that entry into the cytoplasm of the bacterial cell is not necessary for activity. For example, polymyxin B covalently attached to agarose beads has a good antimicrobial activity on $E$. coli and $P$. aeruginosa. ${ }^{94}$ It is proposed that perturbation of outer membrane structure by polymyxin-agarose indirectly affected the selective permeability of the inner membrane and inhibited respiration. However, the chemistry followed in this study did not provide selectivity to the anchoring point on the peptide, given that it includes multiple free amines, any of which could have reacted with the spacer arm bound to the agarose beads. A better example is a cysteinilated derivative of battacin, a close analog of polymyxin that retains its activity when covalently linked to a derivatized solid surface, being a promising agent as antibacterial surface coatings to 
prevent bacterial colonization and biofilm formation. ${ }^{95}$ The lipopeptide-coated surfaces caused significant damage to the cellular envelope of $P$. aeruginosa and $E$. coli upon contact and prevented surface biofilm colonization.

It should be stressed that the mechanism of action of polymyxins on Gram-negative bacteria based on OM and IM interactions is well documented. However, other mechanisms involving intracellular targets can also play a role. ${ }^{15,96}$ Recently, entry of polymyxin into the cytoplasm of Gram-negative bacterial cells has been demonstrated by time-lapse laser scanning confocal microscopy using a dansylated polymyxin $B$ that maintains the pharmacological properties of the natural antibiotic. ${ }^{97}$ In their work, the authors show that labelled polymyxin initially accumulated in the OM of $K$. pneumoniae, then it gradually penetrated the $\mathrm{OM}$ and accessed the IM, and only at high concentrations $(5 \times \mathrm{MIC})$ it became homogeneously distributed in the cytoplasm. The possibility of intracellular targets for polymyxins is not clear, but there are some studies that point in that direction. For example, the generation of hydroxyl radical production by the Fenton reaction leading to the formation of hydroxyl radicals through the reduction of hydrogen peroxide by ferrous ion $\left(\mathrm{Fe}^{2+}\right)$ has been observed in several Gram-negative species, including $A$. baumanii and $E$. coli, ${ }^{76,98}$ and $K$. pneumoniae. ${ }^{99}$ The production of reactive oxygen species (ROS) is concurrent with the rapid killing of these bacteria by both polymyxin $B$ and colistin, probably by oxidative damage in the bacterial DNA, proteins and lipid. ${ }^{100}$ In support of this intracellular mechanism of action, a study by Pournaras et al. ${ }^{101}$ shows that in a colistin-resistant isolate of $A$. baumanii there is a significant decrease in the expression of enzymes involved in oxidative stress response. An increase in expression of genes encoding superoxide dismutase enzymes after colistin treatment in A. baumanii also agrees with the idea of hydroxyl radicals being involved in colistin antibacterial activity. ${ }^{85}$

\section{Resistance to polymyxins}

The therapeutic rescue of polymyxins for their use in nosocomial infections has been followed by an emergence of acquired resistance among the most clinically relevant Gram-negative bacteria. Resistance to polymyxin is a complex subject that would require of another thorough review by itself. Hence, only a brief mention will be made here. Several recent reviews summarizing the mechanisms of resistance to polymyxins are also available. ${ }^{53,102-105}$ As seen before, the first molecular target of polymyxins in the bacterial surface is the LPS of the outer membrane. Since electrostatic interactions are established with anionic phosphate groups in lipid A, modification of those with positively charged groups such as phosphoetanolamine or 4-amino-4-deoxy-L-arabinose provides a mechanism of protection from the interaction with polycationic polymyxin and thus, of resistance. ${ }^{106}$ Changes in lipid A may include deacylation, hydroxylation and palmitoylation. Other mechanisms of resistance include the utilization of efflux pumps and capsule formation. Resistance is mainly adaptive (reversible) and regulated by two-component systems (e. g. PhoP/PhoQ and PmrA/PmrB) and can be triggered by environmental stimuli (low $\mathrm{Mg}^{2+}$ levels, sublethal concentrations of AMPs, for instance). Recently, resistance to colistin due to plasmid-mediated $\mathrm{mcr}-1$ gene has also been described. MCR-
1 is a phosphoethanolamine transferase enzyme (it adds phosphoethanolamine to lipid A). Resistance to polymyxins is certainly an added challenge to the development of new antibiotics against pan-drug resistant Gram-negative bacteria. ${ }^{107}$

\section{Combination with other antibiotics}

The main interest of polymyxin combinations with other antibiotics lies in the treatment of infections caused by resistant and multidrugresistant bacteria whose proliferation is becoming a serious social and economic problem worldwide and account for growing global morbidity and mortality. Multidrug-resistant pathogens are considered those that are resistant to three or more antibiotic classes. The worst are the extensively drug-resistant (XDR) ones, particularly those Gram-negative pathogens that are non-susceptible to all but one or two antibiotic classes. ${ }^{108}$ In this situation, when even carbapenems, a major last-line class of antibiotics to treat bacterial infections, are not useful polymyxins have been rescued and become last-resort agents against XDR $P$. aeruginosa, K. pneumoniae and $A$. baumannii.

The emergence of polymyxin-resistant strains and polymyxin heteroresistance (heterogeneity of response to antibiotics from bacterial cells within the same population) is questioning the utility of polymyxin monotherapies. Increasing the dose to maximize efficacy of the treatment is not viable since polymyxins are nephrotoxic and exhibit a narrow therapeutic index. An alternative option would be the administration of polymyxins in combination with other antibiotic agents or non-antibiotic compounds. ${ }^{109}$ The mechanism of action of polymyxins, that affects the integrity and enhance permeability of the outer membrane of Gram-negative bacteria, may help increase activity and accumulation ${ }^{110}$ within bacterial cell of other antibiotic classes.

Several studies have explored the synergistic activity of polymyxins with other antimicrobial agents against Gram-negative bacteria, particularly $P$. aeruginosa, K. pneumoniae and $A$. baumannii. Synergy may be assessed in vitro by three methods: time-kill studies, Etest and microdilution. The major interest of combinations is to show synergistic activity against resistant bacterial strains to at least one of the antibiotics, chiefly the one showing the highest MIC. One of the antibiotic classes most commonly used in combination with polymyxins is the carbapenem family. In a systematic review and meta-analysis, combination therapy following the time-kill method showed synergy rates of $44 \%$ (30 to 59\%) for K. pneumoniae, $50 \%$ (30 to $69 \%$ ) for $P$. aeruginosa and $77 \%$ (64 to $87 \%$ ) for $A$. baumannii. Of the carbapenems studied, doripenem showed high synergy rates for all three bacteria. Meropenem was more synergistic for $A$. baumannii and imipenem for $P$. aeruginosa. Etest and checkerboard assays generally yielded lower synergy rates than time-kill studies. ${ }^{111}$

In addition to carbapenems, combinations of polymyxins with amikacin, ${ }^{112}$ ceftazidime, ${ }^{113,114}$ ciprofloxacin, ${ }^{114}$ fosfomycin, ${ }^{115-117}$ gramicidin $^{118,119}$ or rifampicin ${ }^{120}$ have been described to confer additive bactericidal activity against several $P$. aeruginosa strains in vitro. Regarding K. pneumoniae, efforts have been mostly devoted to $K$. pneumoniae carbapenemase (KPC) and metallo- $\beta$-lactamase 
(MBL) producing strains. ${ }^{121}$ Synergistic activity has been observed in combinations with fosfomycin, ${ }^{115,122,123}$ vancomycin, ${ }^{124}$ rifampicin, ${ }^{125,126}$ tygecicline, ${ }^{126}$ chloramphenicol, ${ }^{127}$ plazomycin, ${ }^{128}$ and aztreonam. ${ }^{129}$

The combination therapy of polymixin with rifampicin is one of the most tested options for the treatment of MDR and XDR Gramnegative bacterial infections, and $A$. baumannii is no exception. ${ }^{130-134}$ A lot of attention has also received the synergistic studies of polymyxins with glycopeptides (teicoplanin, vancomycin, telavancin). ${ }^{124,135-141}$ Partial synergy has been observed with azithromycin. $^{142}$ Synergistic effectiveness of colistin with meropenem and sulbactam, ${ }^{143}$ minocycline ${ }^{144}$ and daptomycin, an anionic lipopeptide, has also been shown. ${ }^{145}$ Finally, the combination of lantibiotic nisin (normally used as a food preservative) with either colistin or polymyxin $B$ yielded a pronounced synergistic effect in $E$. coli, K. pneumoniae, P. putida and P. aeruginosa. ${ }^{146}$ However, no additive effect was found with nisin in binary combination with penicillin, erythromycin or chloramphenicol.

In summary, numerous studies have identified various polymyxin combinations presenting synergistic activity against sensitive and multi-drug resistant $P$. aeruginosa, K. pneumoniae and $A$. baumannii. Apparently, synergy was more evident in polymyxin-resistant strains, what augurs well for the combination therapy in front of polymyxin monotherapy. In addition, regrowth and emergence of resistance is consistently seen in polymyxin monotherapy. Given this situation, polymyxin combination therapy could reduce the possibility for selection of resistant subpopulations or the development of new resistance. Although most in vitro data endorse this view, clinical investigations of polymyxin combination therapy are in its infancy. So far, clinical data is apparently inconclusive in showing evident superiority of the cotherapy due to the small sample size studies, among other reasons..$^{53,147-149}$ To overcome this issue, well-designed clinical tests are urgently needed to give a clear answer. In this regard, two large clinical trials comparing colistin monotherapy and colistin combined with meropenem are currently in progress both in Europe and the US. ${ }^{150}$

\section{Synergy with antifungals}

Polymyxins are known to have a poor fungicidal activity (MIC $\geq 8$ $\mathrm{mg} / \mathrm{L}$ ). However, the synergistic antifungal properties of polymyxin B were studied as early as 1972 . Polymyxin was found to potentiate the activity of tetracycline in Candida albicans and Saccharomyces cerevisiae, even at low concentrations. Polymyxin B seemed to increase the permeability of the yeast cell membrane to tetracycline, which then inhibited protein synthesis and led to cell death. ${ }^{151}$ More recently, it has been demonstrated that the polymyxin $B$ combination with fluconazole or itraconazole was active at low concentrations against Aspergillus fumigatus, Rhizopus oryzae, Candida albicans and non-albicans Candida species. The combination at clinically relevant low concentrations was particularly potent against Cryptococcus neoformans, including strains resistant to fluconazole. ${ }^{152}$ Polymyxin $B$ has also been demonstrated to reduce the tissue fungal burden both in intravenous and inhalation models of murine cryptococcosis at a level comparable with that of fluconazole. ${ }^{38}$

Synergistic antifungal activity against $C$. albicans has also been reported when polymyxin $B$ was combined with amphotericin B, ketoconazole and miconazole. ${ }^{153,154}$ Similarly, colistin has also been found to act synergistically with amphotericin B against $R$. oryzae. ${ }^{155}$ Colistin, both as a single agent or in combination with voriconazole, caspofungin and amphotericin B, has also shown in vitro antifungal activity against filamentous ascomycetes occurring in cystic fibrosis patients and may offer new therapeutic options, especially for multidrug-resistant Scedosporium prolificans. ${ }^{156}$

In a recent study to assess the in vitro susceptibility of 25 clinical isolates of Fusarium to antifungal agents (amphotericin B, caspofungin, itraconazole and voriconazole) and antimicrobials (pentamidine, polymyxin B, tigecycline and tobramycin), the highest rates of synergism were observed when amphotericin $B$ or voriconazole were combined with tobramycin (80\% and $76 \%$, respectively), polymyxin B (76\% and $64 \%$ ) and pentamidine (72\% and $68 \%) .{ }^{157,158}$

Finally, caspofungin and echinocandin antifungals in combination with colistin have also been found to act synergistically against fluconazole-resistant and susceptible $C$. albicans and $C$. glabrata isolates. However, authors also state that the correlation with in vivo benefits may not be straightforward. ${ }^{159-161}$

\section{Antibiofilm activity}

A biofilm is an organized microbial ecosystem that consist of one or more microbial species imbedded in a self-produced matrix of extracellular polymeric substances composed by proteins, polysaccharides and DNA. Biofilms can develop on human body tissues and surfaces of medical devices. Antibiotic treatments against biofilms usually require of high doses administered for long periods of time. Since current available antibiotics have been developed to target planktonic bacteria, they often fail to fight persistent infections associated with biofilms. ${ }^{162}$

Polymyxins have been proven to be active against biofilms, both as a single agent or in combination with other antibiotics, particularly against $A$. baumannii and $P$. aeruginosa. ${ }^{163,164}$ However, neither colistin nor polymyxin B were found capable of preventing (p)ppGpp accumulation (alarmones guanosine 5'-diphosphate 3'-diphosphate, ppGpp, and guanosine $5^{\prime}$-triphosphate $3^{\prime}$-diphosphate, pppGpp) signaling nucleotides that regulate the stringent response in bacteria and are known to play a role in biofilm formation. ${ }^{165}$

Polymyxin was found to show an antibiofilm synergistic interaction with cyclic antimicrobial peptide gramicidin $\mathrm{S}$ toward 17 multidrugresistant $P$. aeruginosa and biofilms of $P$. aeruginosa strain PAO1. The concentration of polymyxin $B$ required to inhibit biofilm formation by $P$. aeruginosa PAO1 was $8 \mu \mathrm{g} / \mathrm{ml}$. Treatment in combination with gramicidin S required only $2 \mu \mathrm{g} / \mathrm{ml}$ (gramicidin S concentration got reduced from $32 \mu \mathrm{g} / \mathrm{ml}$ to $4 \mu \mathrm{g} / \mathrm{ml}$ ). The FIC (fractional inhibitory concentration) calculated from this decrease 
was 0.375 , which indicated a synergistic effect of this treatment. ${ }^{118}$ Inhibition of biofilm formation by $P$. aeruginosa (PA-01 strain) has also been found between nisin lantibiotic and either colistin or polymyxin B. As a control, none of these antimicrobials inhibited biofilm formation when used individually. ${ }^{146}$

Colistin and tobramycin, both alone or in combination exhibited bactericidal activity prior to biofilm attachment to endotracheal tubes, however no activity was observed once biofilm formed on such polyvinylchloride tubes. ${ }^{166}$ Polymyxin B proved to be $100 \%$ effective in vitro against a highly prevalent clone of multi-drug resistant $A$. baumannii, with a $92.9 \%$ of strains being biofilm producers (associated patient lethality of $28.2 \%$ in a Mexican Tertiary Care Hospital). However, no direct proof of polymyxin inhibiting biofilm formation in this clone was provided. ${ }^{167}$

Recently, colistin entrapped in nanoparticles of different materials [poly (lactide-co-glycolide, chitosan, poly(vinyl alcohol] have been proven to eradicate pre-formed $P$. aeruginosa biofilms. Nanoparticles of colistin/poly(vinyl alcohol) and colistin/chitosan could penetrate inside the biofilms, release colistin in situ, thus increasing the effectiveness of the treatments. ${ }^{168,169} \mathrm{~A}$ synergistic or additive effect between colistin and levofloxacin has been reported in vitro and in Galleria mellonella model against colsitin-susceptible A. baumannii strains, although not against colistin-resistant ones. ${ }^{170}$

\section{Toxicity}

Toxicity of polymyxins has been reported since the beginning of its use. Toxicity is dose dependent and reversible once the treatment is discontinued. However, the exact molecular mechanism of toxicity is not well-understood

In 1947, Stansly and coworkers already described the sulfomethylation of polymyxin to reduce acute toxicity, according to previous reports describing the significance of transforming a cationic drug into an anionic one:

$$
\mathrm{R}-\mathrm{NH}_{2}+\mathrm{HCHO}+\mathrm{NaHSO}_{3} \rightarrow \mathrm{R}-\mathrm{NH}-\mathrm{CH}_{2}-\mathrm{SO}_{3}-\mathrm{Na}^{+}+\mathrm{H}_{2} \mathrm{O}
$$

They also observed that this sulfomethylated form of polymyxin was free of producing painful irritation when administered subcutaneously or intramuscularly. ${ }^{47}$

The main adverse side effects of polymyxins are nephro- and neurotoxicity. ${ }^{171,172}$ The rate of colistin-associated neurological toxicity is approximately $7 \%$. The major manifestation of neurotoxic side effects after parenteral colistimethate administration is paresthesia ("pin and needles sensation"). Neuromuscular blockade can also occur during polymyxin therapy although no episodes have been reported in the literature in the last years. Ataxia, vertigo, confusion, dizziness, weakness, visual, speech disturbances, hallucinations and seizures are also possible during polymyxin therapy.

The major toxicological problem of polymyxins is nephrotoxicity. Both polymyxin B and colistin/colistimethate are known to produce adverse side effects in kidney. ${ }^{171,172}$ Nephrotoxicity rates typically range between $10 \%$ and $60 \%$. In the largest clinical study so far performed (258 patients) the rate of nephrotoxicity was found to be $10 \% \cdot{ }^{173}$ In a series of recent studies carried out in cohorts of 71 and 66 patients, acute kidney injury (AKI) rates associated to colistimethate administration were found to be $45-56 \%$ according to the Risk, Injury, Failure, Loss and End-stage (RIFLE) kidney disease criteria. ${ }^{174,175}$ In a study performed in Korea, AKI incidence was found to reach $54.6 \%$ in a cohort of 119 patients. ${ }^{176}$ In another study involving 92 patients comparing a high-dose of colistimethate treatment (9 MIU, million IU, followed by a maintenance dose of 4.5 $\mathrm{MIU} / 12 \mathrm{~h}$ ) versus the standard dose (6 MIU/day), a higher cure rate in the high-dose group was found ( 63 vs. $41.3 \%, p=0.04$ ) with no higher risk of nephrotoxicity (32.2 versus $26 \%$ ). ${ }^{177}$ In a smaller study involving 30 patients in Orlando (Florida, US), nephrotoxicity occurred in 3 out of 10 (33\%) of patients. An interesting point of this study was that excessive colistimethate dosing was frequent $(47 \%)$, often due to the higher body weight in obese patients (71\%), and was associated with higher rates of nephrotoxicity ( $80 \%$ versus $30 \%$ ). Overall, a close monitoring of the renal function in patients is mandatory to identify the optimal colistimethate dose from both efficacy and toxicity perspectives. ${ }^{178}$ The use of homogeneous criteria, such as the RIFLE one, when carrying out nephrotoxicity studies was also found to be necessary for the evaluation of acute kidney injury and allow for comparison of nephrotoxicity among reports.

The use of potentially nephroprotective agents has been advocated to ameliorate adverse side effects of polymyxins (ascorbic acid, vitamin $\mathrm{E}$, melatonin, lycopene, $\mathrm{N}$-acetylcysteine). In rodent in vivo models, some positive results showed that coadministration of antioxidants can protect against colistin-induced nephrotoxicity. ${ }^{179}$ In the clinic, administration of intravenous ascorbic acid has been studied as a potentially useful component to prevent nephrotoxicity because of its antioxidant properties. However, evidence of a protective effect is not conclusive according to two clinical studies. ${ }^{180,181}$

Since both polymyxins and colistimethate are nephrotoxic, a question rises regarding the relative toxicity of the free base in comparison with the methansulfonated derivative. Again, results are not conclusive. In a report by Oliveira in Brazil dealing with the treatment of infections (41 patients) caused by carbapenemresistant Acinetobacter spp, both polymyxins gave equivalent results with regard to efficacy and toxicity. ${ }^{177}$ In a systematic review and meta-analysis, unadjusted nephrotoxicity was more common in patients treated with colistin than with polymyin B, but according to the RIFLE criteria, there was no difference regarding risk, injury or failure between colistin and polymyxin B. ${ }^{183}$ Two other studies though, reported that nephrotoxicity rates were lower with polymyxin B than colistimethate. ${ }^{171,184,185}$ Also, polymyxin B seems to have some advantage over colistimethate regarding rapid target concentration attainment and antibacterial activity. ${ }^{186}$

\subsection{Mechanism of nephrotoxicity}

The mechanism of polymyxin-induced nephrotoxicity is not clear and numerous efforts are carried out to uncover it. ${ }^{187,188}$ The structureactivity data on polymyxin analogs seem to indicate that toxicity is 
related to the amphipathic nature of the molecule, due to the presence of hydrophobic residues (fatty acid, amino acids 6 and 7) and particularly due to the presence of charged Dab side chains at physiological $\mathrm{pH}$. Polymyxins accumulate within proximal tubular epithelial cells (PTEC) of the kidney via endocytosis at the multiligand receptor megalin. Megalin is highly expressed on the luminal surface of renal proximal tubules. It is a negatively charged protein involved in the binding and endocytosis of polybasic molecules such as aprotinin, cytochrome cor aminoglycosides. ${ }^{189,190}$ The mechanism of nephrotoxicity of polymyxins has been proposed to be similar to that described for aminoglycosides. ${ }^{191,192}$

Polymyxin-induced nephrotoxicity has been associated with acute tubular necrosis in kidneys and rise of creatinine levels in blood. Serum creatinine increments after parenteral administration of colistimethate are dose- and duration-dependent as proven in rats and humans. Phamacokinetic data indicate that polymyxins are extensively reabsorbed by active transport processes in the renal tubules after filtration at the glomerulus (from tubular urine back into blood). ${ }^{193}$ This major tubular reabsorption may induce an accumulation of the drug in the tubular cells and this could be, at least in part, a potential cause for nephrotoxicity. Accumulation of polymyxin induces apoptosis in kidney tubular cells ${ }^{194}$ and may be the result of mitochondrial damage and/or release of reactive oxygen species. ${ }^{187}$ The urinary recovery of polymyxin $\mathrm{B}$ and colistin is less than $1 \%$, as proven in humans and rats, respectively. Thus, it is evident that nonrenal elimination is the predominant clearance pathway for both polymyxins. However, the urinary recovery of sodium colistimethate can be higher than $60 \%$ (as seen in assays in rats, dogs and humans). The explanation may come from the different ionic nature of both polymyxins (polycations) and colistimethate (a polyanion). Urinary concentrations of colistin after administration of colistimethate can be relatively high as a result of hydrolysis within the urinary tract from colistimethate that is extensively renally excreted (and not reabsorbed). Partially sulphomethylated derivatives of colistin are also excreted before the full hydrolysis and are not reabsorbed. ${ }^{195-197}$

Recently, a detailed study by Mass Spectrometry Imaging (MSI) helped to reveal the distribution of polymyxin B1, colistin, and the less nephrotoxic truncated derivative, polymyxin B nonapeptide within rat kidney. ${ }^{198}$ The bioanalysis showed that polymyxin B1 and colistin preferentially accumulated in the renal cortical region while polymyxin B nonapeptide, a less nephrotoxic compound, was more uniformily distributed throughout the kidney. Ratios of relative proportions of kidney cortex:medulla at seven days were 5.8 to 1

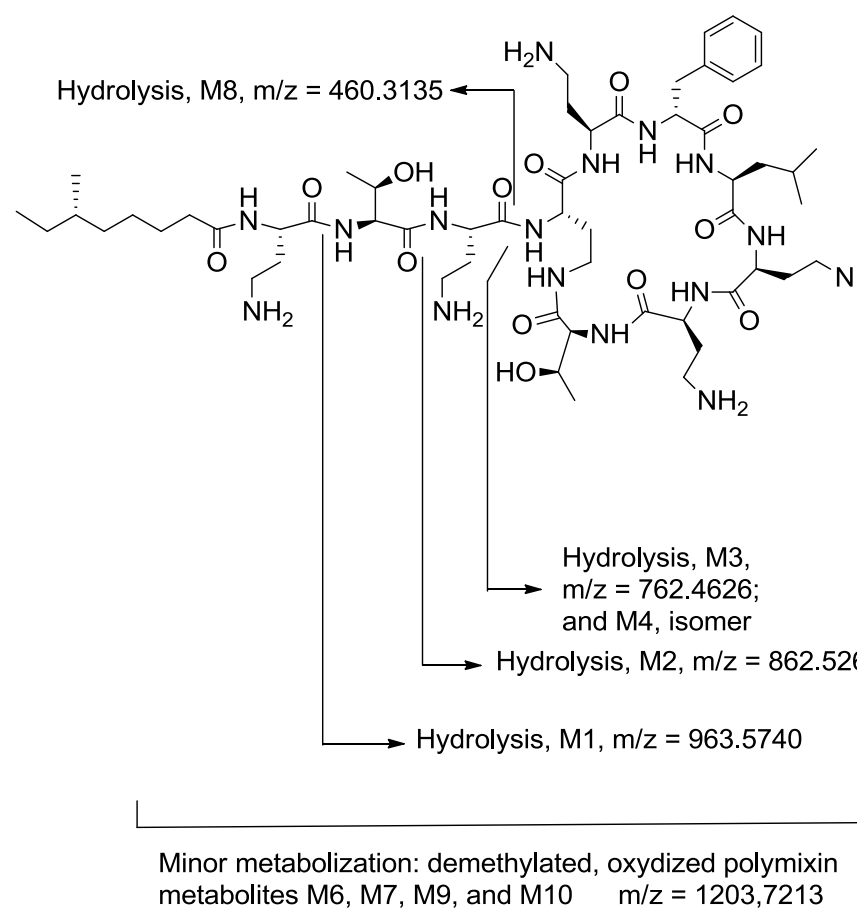

Figure 4: Polymyxin B1 metabolites found in renal cortex in addition to polymyxin B1 itself. Metabolites were identified by LC/MS/MS (Liquid Chromatography, Mass Spectrometry) according to Nilsson and coworkers (M5, unknown structure). Arrows indicate peptide bonds metabolized by hydrolysis. ${ }^{198}$

for colistin and 25.4 to 1 for polymyxin B. For polymyxin nonapeptide, the ratio was 2.9 to 1 . These results correlate with recent immunohistochemical and correlative microscopy studies, which also demonstrated that polymyxins accumulate within renal tubular cells of the renal cortex. ${ }^{199-202}$ In addition, metabolites that accumulate with a similar distribution as the parent polimixins have also been identified by high resolution MS (Figure 4).

Both tissue homogenates and urine samples were analyzed. Ten polymyxin metabolites were identified within the tissue homogenates, six of which were also found in the urine sample. No quantification of the relative abundances of the different metabolites was provided, though. Nevertheless, it is interesting to see that both non-metabolized polymyxin and colistin accumulate in renal cortex and their image intensity increases during the seven days of the experiment. Some metabolites imply minor modification (oxidation, demethylation) of the parent polymyxin, with small reductions of molecular weight. Other metabolites comprise the sequential hydrolysis of the linear lipotripeptide moiety without affecting the cyclic heptapeptide structure. Regarding colistin metabolization, similar byproducts were found. However, one of the metabolites detected involved the partial hydrolysis of cyclic structure in this case (colistin metabolite M5, not shown). ${ }^{198}$ Altogether, this study suggests that in spite of the fact that the majority of kidney's drug metabolizing and detoxification enzymes are located in the proximal tubule, ${ }^{203}$ a significant proportion of polymyxin molecules remain non-metabolized in renal cortex, what seems to indicate that polymyxins are apparently quite stable 
molecules. In fact, polymyxins accumulate and are reabsorbed in proximal tubules, as we have seen before, and are mainly eliminated by non-renal clearance mechanisms.

\subsection{Biomarkers of nephrotoxicity}

Monitoring of drug-induced kidney injuries is an important issue during drug development. Hence, validation of kidney injury biomarkers is necessary for the clinical application and regulatory issues. In acute kidney injury according to the RIFLE criteria, the determination of plasma and serum creatinine levels remains as a standard of diagnosis. Creatinine is a useful biomarker of kidney damage in patients suffering of stable chronic renal diseases, but it is not convenient in the case of acute kidney disease. More than half of kidney function is lost before AKI is detected by an increase of serum creatinine levels as they are detected 24-48 hours following the initial kidney insult. Colistimethate treatments are often discontinued when minor increments in creatinine levels are detected because of the risk of negative outcomes, even if patients are responding to the antibiotic therapy. Hence, there is a need of novel kidney injury urinary biomarkers that can detect AKI much earlier in order to avoid such decision time lag. Several biomarkers are a subject of study to allow for an early and site-specific prediction of AKI. These indicators can detect initial kidney injury in 4-6 hours. Biomarkers for tubular damage include Kidney Injury Molecule-1 (KIM-1), $\alpha$-Glutathione S-Transferase $(\alpha-G S T), \pi$-Glutathione STransferase $(\pi-G S T)$ and NGAL (Neutrophil Gelatinase Associated Lipocalin). ${ }^{197,204,205}$

KIM-1 is a type I trans-membrane structural glycoprotein present on epithelial cells of the renal proximal tubule. KIM-1 is undetectable in healthy tissue but highly upregulated on the apical surface of proximal tubule epithelial cells after renal injury, i. e. in response to toxic and ischemic injury. The ectodomain shedding of KIM-1 into urine makes it an early and specific biomarker for AKI.

$\alpha-$ GST and $\pi-$ GST are small cytosolic isoenzymes and members of the GST superfamily. They are primarily involved in cellular detoxification metabolic reactions since they catalyze the conjugation of the reduced form of glutathione (L- $\gamma$-glutamyl-Lcysteinyl-glycine) to xenobiotic substrates. They can form up to $2 \%$ of the total cytosolic soluble protein content in the cytoplasm. $\alpha$-GST and $\pi$-GST are immediately released into the urine upon lysis of epithelial cells of the proximal and distal tubule, respectively, converting them in a rapid and sensitive biomarker of AKI. Simultaneous detection of both GST proteins permits discrimination between proximal and distal tubular damage.

NGAL is small extracellular glycoprotein that is characterized by the ability to bind small hydrophobic molecules. NGAL is rapidly upregulated and thus, an early biomarker for the detection of AKI onset in various clinical settings including cardiothoracic surgery, intensive care-unit, and nephropathy.

In a study carried out in rats, KIM-1 and $\alpha$-GST proved to be the most sensitive biomarkers to polymyxin-induced AKI, outperforming current nephrotoxicity standards of care (creatinine and blood urea nitrogen) proving that they are not suitable for detecting nephrotoxicity of polymyxin in this in vivo model. ${ }^{197}$ NGAL and $\pi-G S T$ also proved to be useful indicators according to same study. In another in vivo study performed simultaneously in rat, dog and monkey, similar results were obtained for the rat model, with NGAL and KIM-1 capable of detecting AKI (GST biomarkers were not tested). However, in the dog and monkey models, both standard (creatinine and blood urea nitrogen) and more recent biomarkers (NGAL and KIM-1) showed a rapid onset of response. ${ }^{204}$ In this case, though, the added value of urinary NGAL and urinary KIM-1 biomarkers was their selectivity in the localization of the injury within the kidney (proximal and/or distal tubules versus the glomerulus) and can help to discriminate the development of acute kidney injury onset. $^{205}$

\section{Design and development of new polymyxins}

The growing incidence of bacterial resistance in hospitals and healthcare settings, the dry pipeline of new antibiotics, and the fact that polymyxin B and colistin have become last line antibiotics to treat highly drug resistant infections in spite of their toxicity liabilities has led to several worldwide research initiatives in recent years to design and develop new polymyxin analogs. The goals are to improve activity, reduce adverse side effects, mostly nephrotoxicity, and understand the relationship of activity and toxicity with the chemical structure of polymyxins.

First efforts aiming at these objectives started in the 1970s. However, the difficulty in access to reliable peptide synthesis schemes for the preparation of cyclic peptide analogs and the limited knowledge of polymyxin pharmacology reduced the structural and chemical space of explored analogs. ${ }^{14,15,206-210}$ Thus, compounds were generated by acylation or alkylation of Dab residues, or substitution of the $\mathrm{N}$ terminal fatty acid tail sometimes following semisynthetic approaches from truncated versions of polymyxin such as the nonapeptide (PBNP). The view that emerges from this initial background is that amphipathicity in polymyxins is crucial for activity, which includes the charged Dab residues on the one hand and the fatty acid tail and the conserved hydrophobic residues in position 6 and 7 on the other.

Another trend that can be observed relates to the fact that in general, activity in polymyxins runs parallel to toxicity, i. e. more active compounds tend to be more toxic to mammalian cells. Hence, new approaches should be sought to break this activity/toxicity correlation in polymyxins. In the last decade or so, several academic and private teams worldwide got involved in the design and development of novel polymyxin analogs to overcome the drawbacks posed by the natural compounds, that is to say, improve safety and efficacy.

\subsection{Synthetic preparation of polymyxin analogs}

Preparation of novel polymyxin analogs is performed following two main approaches: semisynthesis and total chemical synthesis. The first strategy, semisynthesis, starts from the natural polymyxin product, usually obtained by fermentation procedures. ${ }^{211}$ Although this is a readily accessible and affordable approach, it is curtailed by the few possibilities of modifying the cyclic heptapeptide core and 
normally analogs are reduced to truncation or substitutions in the linear lipotripeptide moiety by enzymatic treatment. Enzymatic hydrolysis of the linear segment of polymyxin gives access to truncated analogs of polymyxin, such as the deacylated decapeptide by means of a polymyxin acylase treatment or to the well-known PBNP by treatment with papain or plant proteinase ficin. ${ }^{202-203}$ Treatment with bromelain enzyme yields polymyxin octapeptide (polymyxin 3-10) while hydrolysis with Nagarse furnishes the heptapeptide (polymyxin 4-10, heptacycle devoid of the N-teminal lipotripeptide). ${ }^{210-211}$ Further elaboration of the polymyxin fragments is possible by means of several selective protection schemes (Boc, tert-butoxycarbonyl, Fmoc, 9-fluorenylmethoxycarbonyl) and strategies as discussed below for each particular case.

Total chemical synthesis of polymyxins generally involves the solidphase methodology using different polymeric supports and protecting groups such as Fmoc, Boc, tBu (tert-butyl), Dde (1-(4,4dimethyl-2,6-dioxocyclohex-1-ylidene)ethyl), z (benzyloxycarbonyl) or Bzl (benzyl). Below, efforts by private companies and universities devoted to the design and development of polymixins are summarized.

\subsection{CB-182,804 analog}

This was the first synthetic polymyxin B derivative that entered a clinical trial. Originally developed by BioSource Pharm and licensed to Cubist Pharmaceuticals (now part of Merck), it had its N-terminal fatty acyl moiety substituted by an aromatic urea, a 2-chlorophenylaminocarbonyl unit (Figure 5).

The analog CB-182,804 was prepared by semisynthesis from natural polymixin obtained by fermentation. Side chain Dab amino groups were protected with the Sulfmoc group $\left(\mathrm{HSO}_{3}-\mathrm{Fmoc}\right)$ by reaction with 9-(2-sulfo)fluorenylmethoxycarbonyl-N-hydroxysuccinimide. The resulting penta-Sulfmoc protected polymyxin was treated with a deacylase enzyme (from Actinoplanes ulahensis NRRL 12052) to remove the fatty acid tail and the resulting $\mathrm{N}$-terminal free amino group was reacted with 2-chlorophenylisocyanate to yield the o-chlorophenyl urea. Finally, the Sulfmoc protecting groups were removed with piperidine in methanol to obtain the expected polymyxin derivative. ${ }^{212}$

In vitro activity of CB-182,804 showed MIC90 (minimal concentration to inhibit growth of $90 \%$ of bacteria strains) values for $E$. coli, $K$. pneumoniae and $P$. aeruginosa around $2-4 \mu \mathrm{g} \cdot \mathrm{mL}^{-1}$, similar or slightly higher (less potent) than polymyxin $\mathrm{B} /$ colistin. ${ }^{212,213}$ It was also demonstrated to be substantially less toxic in vitro since an $\mathrm{EC}_{50}$ of more than $1000 \mu \mathrm{g} \cdot \mathrm{mL}^{-1}$ was obtained in rat kidney proximal tubule cell cultures whereas $\mathrm{EC}_{50}$ was $318 \mu \mathrm{g} \cdot \mathrm{mL}^{-1}$ for polymyxin $\mathrm{B}$.

CB-182,804 was tested in eight rodent infection models for efficacy against five Gram-negative pathogens. The efficacy of CB-182,804, for instance, compared favorably to that of polymyxin B, colistin, ciprofloxacin, or imipenem-cilastatin in an in vivo model of $P$. aeruginosa lung infection in neutropenic mice.

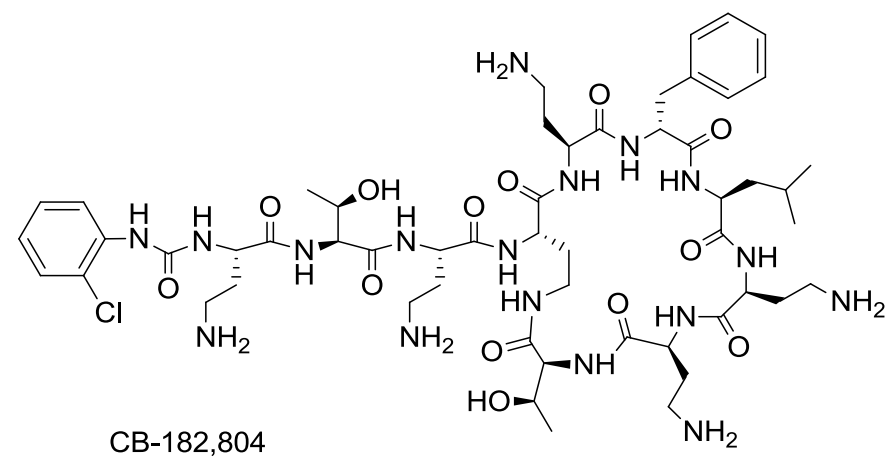

Figure 5: Chemical structure of analog CB-182,804

Similarly, in an A. baumannii thigh infection model in neutropenic mice, it also compared favourably to polymyxin B, colistin or imipenem-cilastatin. Pharmacokinetic features showed differences with respect to polymyxin $B$, particularly, decreased serum protein binding, and increased plasma clearance and volume of distribution.

Finally, CB-182,804 was tested in cynomolgus monkeys for renal toxicity in a seven-day study. CB-182,804 showed lower nephrotoxicity than polymyxin $B$. Histopathological changes were characterized by minimal to mild degeneration/necrosis $(6.6$ $\mathrm{mg} / \mathrm{Kg} /$ day dose). ${ }^{212}$

There was a mild associated increase in blood urea nitrogen and serum creatinine at higher dose with an increase in severity of histological kidney changes $(9.9 \mathrm{mg} / \mathrm{Kg} /$ day $)$. However, these kidney findings were markedly less than the ones found for polymyxin $B$ when administered at equivalent antimicrobial doses. CB-182,804 progressed into a phase I clinical trial (safety and pharmacokinetics in healthy humans) in February 2009 but in September 2010, it was discontinued. Results have apparently not been published.

\subsection{Pfizer 5x analog}

A series of biaryl and heterobiaryl N-terminal substituted derivatives of polymyxin have been described by Magee and coworkers in Pfizer. ${ }^{214}$ Position 3 that usually contains a Dab amino acid in natural polymyxins was substituted by Dap (L-1,3-diaminopropanoic acid) in the analogs (Figure 6). This substitution conferred a higher antimicrobial activity and reduced cytotoxicity in human PTEC cells. Compounds were prepared by solid phase chemical synthesis on a 2-chlorotrityl chloride resin following a Fmoc/tBu/Z/Dde scheme of protection and macrocyclization in solution at high dilution conditions $(0.002 \mathrm{M})$. The heterobiaryl moiety was introduced by acylation reaction of 6-oxo-1-phenyl-1,6-dihydropyridine-3carboxylic acid. Antimicrobial potency of $5 x$ was similar to the one of polymyxin $\mathrm{B}$, as judged by $\mathrm{MIC}_{50}$ and $\mathrm{MIC}_{90}$ (equal or half the value) against susceptible and resistant strains of $P$. aeruginosa, $A$. baumannii, E. coli and K. pneumoniae. 


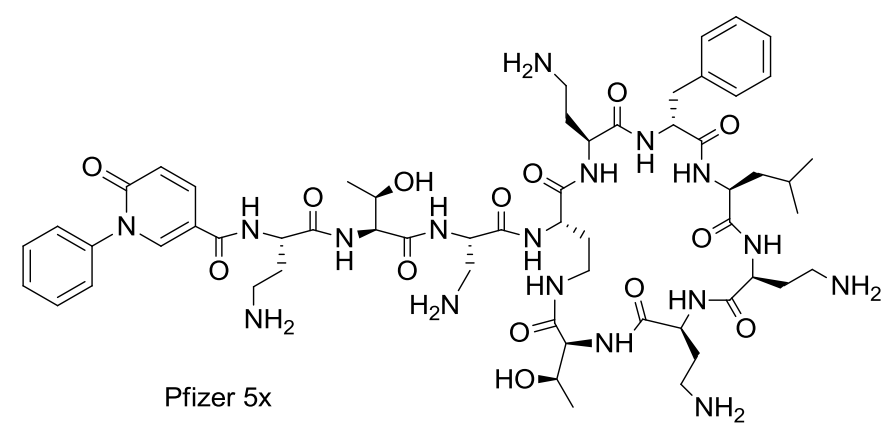

Figure 6: Chemical structure of Pfixer 5x analog

Compound $5 x$ showed improved cytotoxicity in human PTEC cell line $\left(\mathrm{TC}_{50}>100 \mu \mathrm{M}\right)$ in comparison with polymyxin $\mathrm{B}\left(\mathrm{TC}_{50}>22 \mu \mathrm{M}\right)$. A extensive comparative in vivo safety study in rat and dog models was performed. In a seven-day exploratory toxicological study, $5 x$ showed no incidence of necrotic kidney lesions at $4 \mathrm{mg} / \mathrm{Kg} /$ day dose while polymyxin $B$ yielded necrotic kidney lesions in every animal although graded to be minimal. At twofold dose ( $8 \mathrm{mg} / \mathrm{Kg} /$ day $), 5 x$ produced no kidney lesions whereas polymyxin $B$ was not even tolerated. However, the dog model proved to be more sensitive to this seven-day exploratory toxicological study. Although at a low dose of $5 \mathrm{mg} / \mathrm{Kg} /$ day was well tolerated, minimal nephrotoxicity signs were already observed in all eight animals tested. At higher doses (11 and $20 \mathrm{mg} / \mathrm{Kg} /$ day) histopathology revealed moderate to marked nephrotoxicity signs. Although the severity of renal lesions was higher for polymyxin $B$, the authors conclude that there was no significant safety margin in dog and further preclinical development was not pursued. This result also demonstrates that the broad in vitro difference in cytotoxicity observed in the PTEC assay in favor of $5 x$ versus polymyxin $B$ did not correlate with the in vivo safety test in dog. Thus, authors conclude that further work needed to be carried out to develop in vitro assays that are able to predict nephrotoxicity in preclinical models, particularly non-rodent ones. ${ }^{214}$

\subsection{Monash FADDI analogs}

The Monash Institute of Pharmaceutical Sciences has also addressed the design of new polymyxin analogs focusing mainly in the hydrophobic domains of the molecule, residues 6 and 7 in the heptapeptide cycle and the fatty acid tail. Compounds were prepared by total chemical synthesis on solid phase. A Fmoc/tBu/Dde scheme of protection on a trityl resin was used. Macrocyclization took place in solution at high dilution conditions. Design of the analogs incorporated new hydrophobic moieties such as octylglycine (L-2-aminodecanoic acid) and biphenylalanine that resulted in a substantial improvement of in vitro potency against polymyxin resistant isolates. ${ }^{215}$

FADDI-002 and FADDI-003 (Figure 7) yielded MIC of 2-4 $\mu \mathrm{g} \cdot \mathrm{mL}^{-1}$ against polymyxin-resistant strains of $P$. aeruginosa $(32$ to $>32$ $\mu \mathrm{g} \cdot \mathrm{mL}^{-1}$ for polymyxin B) and A. baumannii (2-16 $\mu \mathrm{g} \cdot \mathrm{mL}^{-1}$ in front of 8-128 $\mu \mathrm{g} \cdot \mathrm{mL}^{-1}$ for polymyxin B). Against polymyxin sensitive strains, though, FADDI analogs were slightly less potent compared to polymyxin and colistin (MIC 1-4 $\mu \mathrm{g} \cdot \mathrm{mL}^{-1}$ compared to 0.5-2 $\mu \mathrm{g} \cdot \mathrm{mL}^{-1}$ ). Activity against Gram-positive bacteria was also reported and resistant strains of Staphylococcus aureus and Enterococcus faecium rendered MIC of 4-16 $\mu \mathrm{g} \cdot \mathrm{mL}^{-1}$.
FADDI-002 R $=\mathrm{CH}_{3}\left(\mathrm{CH}_{2}\right)_{6^{-}}, \quad \mathrm{R}_{3}=\mathrm{NH}_{2} \mathrm{CH}_{2} \mathrm{CH}_{2^{-}}$ FADDI-003 R= 4-Ph-Ph-,$\quad \mathrm{R}_{3}=\mathrm{NH}_{2} \mathrm{CH}_{2} \mathrm{CH}_{2}-$ FADDI-100 R $=\mathrm{CH}_{3}\left(\mathrm{CH}_{2}\right)_{6}-, \mathrm{R}_{3}=\mathrm{NH}_{2} \mathrm{CH}_{2} \mathrm{CH}_{2^{-}}$ FADDI-287 R= $\mathrm{CH}_{3}\left(\mathrm{CH}_{2}\right)_{6}-, \mathrm{R}_{3}=\mathrm{NH}_{2} \mathrm{CH}_{2}^{-}$

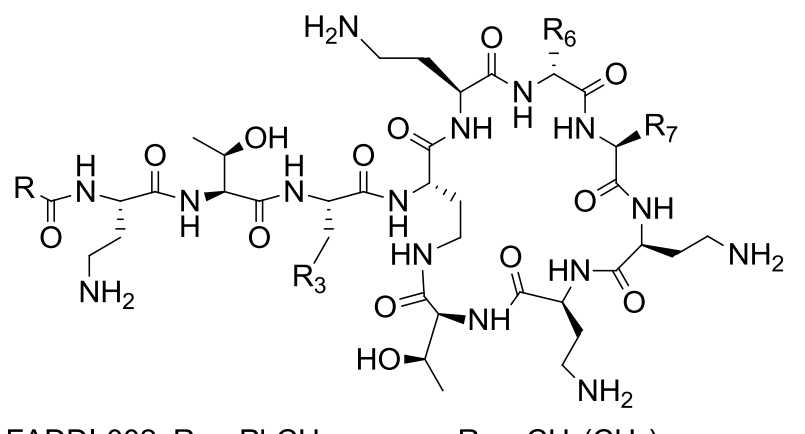

$$
\begin{array}{ll}
\text { FADDI-002: } \mathrm{R}_{6}=\mathrm{PhCH}_{2^{-}}, & \mathrm{R}_{7}=\mathrm{CH}_{3}\left(\mathrm{CH}_{2}\right)_{7^{-}} \\
\text {FADDI-003: } \mathrm{R}_{6}=\mathrm{PhCH}_{2^{-}}, & \mathrm{R}_{7}=\mathrm{CH}_{3}\left(\mathrm{CH}_{2}\right)_{7^{-}} \\
\text {FADDI-100: } \mathrm{R}_{6}=\left(\mathrm{CH}_{3}\right)_{2} \mathrm{CHCH}_{2^{-}}, \mathrm{R}_{7}=\mathrm{CH}_{3}(\mathrm{OH}) \mathrm{CH}^{-} \\
\text {FADDI-287: } \mathrm{R}_{6}=\left(\mathrm{CH}_{3}\right)_{2} \mathrm{CHCH}_{2^{-}}, \mathrm{R}_{7}=\mathrm{CH}_{3} \mathrm{CH}_{2^{-}}
\end{array}
$$

Figure 7: Chemical structure of Monash FADDI analogs

In an in vivo mouse lung infection model, FADDI-002 demonstrated better efficacy than colistin against a polymyxin resistant clinical isolate of $P$. aeruginosa. Regarding safety and tolerability, no adverse effects were detected when a bolus dose of FADDI-002 was administered in rats (intravenous, $0.75 \mathrm{mg} / \mathrm{kg}$ ) and mice (subcutaneous, $40 \mathrm{mg} / \mathrm{kg}$ ). These in vivo preliminary results suggested that the FADDI series had at least a comparable tolerability to polymyxin in rodents. Further exploration led to substitution of position 7 with a threonine (analog FADDI-100), reminiscent of polymyxin M. FADDI-100 rendered a reduction of nephrotoxicity but when tested for potency in a collection of $250 P$. aeruginosa isolates from Rempex-The Medicines Company, a $\mathrm{MIC}_{90}$ of $32 \mu \mathrm{g} \cdot \mathrm{mL}^{-1}$ was obtained. ${ }^{216}$ Finally, FADDI-287, a polymyxin E3 analog with position 7 substituted by acid L-2-aminobutanoic (Abu) and Dap in position 3 (like analog $5 x$ reviewed before), provided high potency ( $\mathrm{MIC}_{90}=1$ $\mu \mathrm{g} . \mathrm{mL}^{-1}$ in the same collection of $P$. aeruginosa isolates; $\mathrm{MIC}_{90}=0.5$ $\mu \mathrm{g} \cdot \mathrm{mL}^{-1}$ against 210 A. baumannii isolates). In vivo kidney histopathology in mouse model (dose at $12 \mathrm{mg}$ base/ $\mathrm{Kg}$, subcutaneously, every $2 h \times 6$ ) showed much lower level of nephrotoxicity (mild acute tubular damage with tubular dilation) than polymyxin B (severe acute tubular damage and cortical necrosis). Efficacy test in mice were also encouraging. ${ }^{216}$

\subsection{Queensland analogs}

Cooper's group at the University of Queensland has been involved in the design and preparation of polymyxins and octapeptins. In an article by Gallardo-Godoy and coworkers, they performed a systematic activity-cytotoxicity study involving the preparation of 32 analogs probing eight of the amino acid positions in polymyxin (Figure 8). ${ }^{217}$ Compounds were prepared by solid-phase chemical synthesis following a Fmoc/tBu/allyl scheme of protection on DHP polystyrene resin (3,4-Dihydro-2H-pyran-2-yl-methoxymethyl polystyrene) with side chain anchoring of the C-terminal threonine. Selective Dab modification was also achieved with the use of ivDde (1-(4,4-dimethyl-2,6-dioxocyclohex-1-ylidene)isovaleryl) protecting group when necessary. 


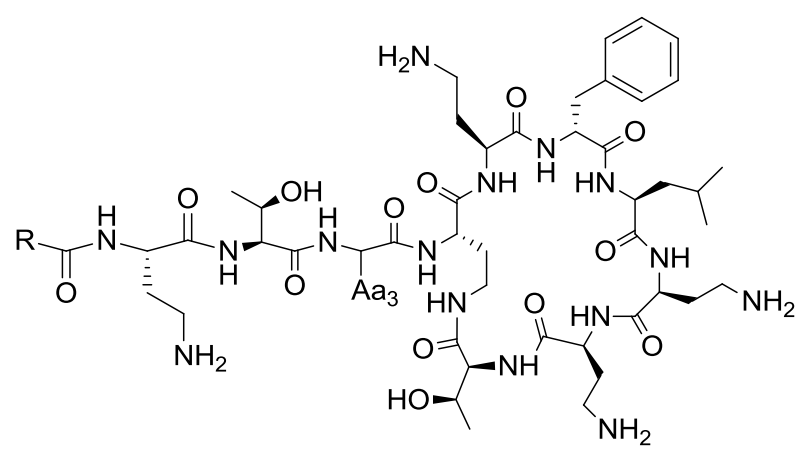

Queensland analog 38, Aa3= Gly, $\mathrm{R}=\mathrm{CH}_{3}\left(\mathrm{CH}_{2}\right)_{6}{ }^{-}$

Queensland analog 10, Aa3=Dab, R= 4-Ph-Ph-

Queensland analog 14, Aa3=Dab, R= 4-PhO-Ph-

Figure 8: Chemical structure of University of Queensland analogs ( $\mathrm{Aa}$ corresponds to the side chain of the indicated amino acid).

MIC results confirmed that more lipophilic side chains at positions 6/7 improved activity against polymyxin-resistant strains but increased cytotoxicity in mammalian cells. Substitution of the Dab residues rendered different behavior depending on the position. Dab-5 and Dab-9 could not be replaced without severely affecting potency, while Dab-8, Dab-3 (substituted by Gly, Thr, D-Dab or D-Ser, the last two reminiscent of polymyxins $A, D, S$ or $P$ ) and Dab-1 tolerated some substitutions.

Another interesting result of the study was the nephrotoxicity predictive potential of in vitro cytotoxicity tests, an issue that was also discussed at Pfizer's work with analog 5x. ${ }^{214}$ Polymyxin B and colistin cytotoxicity were tested using cell lines HepG2 (hepatocyte carcinoma cells) and HEK293 (embryonic kidney cells). Results showed an apparent lack of toxicity $\left(\mathrm{CC}_{50}>300 \mu \mathrm{M}\right)$ whereas in primary renal cell assay measuring LDH (Lactate Dehydrogenase) and GGT (Gamma Glutamyl Transferase) release, toxicity was clearly evidenced $\left(\mathrm{CC}_{50}\right.$ of $\left.23-177 \mu \mathrm{g} / \mathrm{mL}\right) .{ }^{217}$ An opposite behavior was observed with analog 14 of the series that yielded some toxicity readout in cell lines $(287-296 \mu \mathrm{M})$ while in primary cultures gave a $\mathrm{CC}_{50}$ of $>128 \mu \mathrm{g} / \mathrm{mL}$.

\subsection{Northern Antibiotics analogs}

The team of Vaara at Northern Antibiotics in Helsinki has described a series of polymyxin analogs carrying only the three Dab residues. Compounds are obtained by substituting one or two of the Dab residues by D-Thr, D-Ser or Abu (2-aminobutanoic acid) amino acids, shortening the length of the linear tripeptide moiety of natural polymyxin, or both. The reason for this design lies in the fact that reducing the cationic character by eliminating some of positive charges in the molecule may also lead to a reduction in nephrotoxicity (Figure 9). Compounds of this family have direct antimicrobial activity (NAB739 NAB815) or sensitizing activity with other antibiotics (NAB741 and NAB7061). Analogs were synthesized following conventional solid phase chemical methods using a Fmoc/Bzl/Boc strategy of protection. Cyclization was carried out in solution. ${ }^{16}$ Analog NAB739 is based on polymyxin B but the Nterminal lipotripeptide has been substituted by an octanoyl-Thr-DSer segment. NAB739 exhibited good antimicrobial potency against Enterobacteriaceae comparable to the one of polymyxin $\mathrm{B}$.

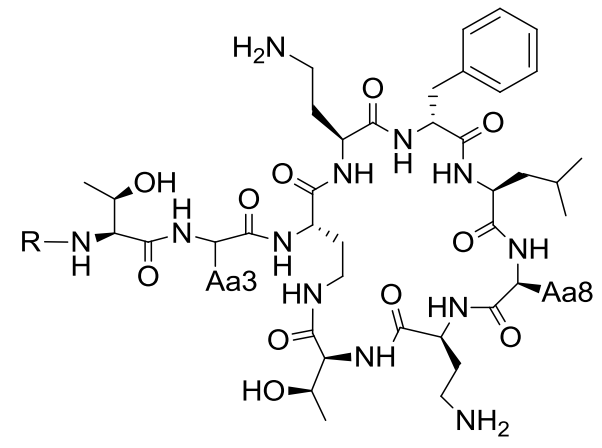

NAB739: $\quad \mathrm{Aa} 3=\mathrm{D}-\mathrm{Ser}, \mathrm{Aa} 8=\mathrm{Dab}, \mathrm{R}=\mathrm{CH}_{3}\left(\mathrm{CH}_{2}\right)_{6}$-CO-

NAB815: $\mathrm{Aa} 3=\mathrm{D}-\mathrm{Thr}, \mathrm{Aa} 8=\mathrm{Abu}, \mathrm{R}=\mathrm{CH}_{3}\left(\mathrm{CH}_{2}\right)_{6}-\mathrm{CONH}-\mathrm{Dab}$

NAB741 Aa3=D-Ser, Aa8=Dab, $\mathrm{R}=\mathrm{CH}_{3}-\mathrm{CO}-$

NAB7061: $\mathrm{Aa} 3=\mathrm{Abu}, \quad \mathrm{Aa} 8=\mathrm{Dab}, \mathrm{R}=\mathrm{CH}_{3}\left(\mathrm{CH}_{2}\right)_{6}$-CO-

Figure 9: Chemical structure of Northern Antibiotics analogs (Aa corresponds to the side chain of the indicated amino acid).

Against E. coli, a concentration of $1 \mu \mathrm{g} / \mathrm{mL}$ of NAB739 inhibited growth in $74.5 \%$ of the strains while polymyxin B inhibited $84.3 \%$ of the strains in the same conditions. Against $K$. pneumoniae, the values of $\mathrm{MIC}_{90}$ for NAB739 and polymyxin B were 2 and $1 \mu \mathrm{g} / \mathrm{mL}$, respectively. However, NAB739 was not so active against $A$. baumannii ( $\mathrm{MIC}_{90}$ was $8 \mu \mathrm{g} / \mathrm{mL}$, four-fold that of polymyxin B), $P$. aeruginosa ( $\mathrm{MIC}_{90}$ was $16 \mu \mathrm{g} / \mathrm{mL}$, eight-fold) and polymyxin-resistant strains. ${ }^{218,219}$ In addition to antibacterial activity, NAB739 displayed sensitizing activity at subinhibitory concentrations, facilitating the penetration of other antibiotics into bacterial cells. Against $A$. baumannii and at a concentration of $0.5 \mu \mathrm{g} / \mathrm{mL}$, NAB739 had synergistic activity and reduced the MIC of rifampicin (from 4-12 to $0.05-0.1 \mu \mathrm{g} / \mathrm{mL}$ ), clarithromycin (from $6-8$ to $0.5 \mu \mathrm{g} / \mathrm{mL}$ ) and vancomycin (from 256 to $3 \mu \mathrm{g} / \mathrm{mL}$ ). ${ }^{220-222}$ Cytotoxicity of NAB739 proved to be 26 -fold less toxic than polymyxin B in HK-2 (human renal proximal tubular) cells with a $\mathrm{CC}_{50}$ of 337 and $13 \mu \mathrm{g} / \mathrm{mL}$, respectively (colistin's was $45 \mu \mathrm{g} / \mathrm{mL}$ ). In permeabilized (electroporated) LLC-PK1 (porcine renal proximal tubular) cells, polymyxin $B$ induced necrosis at $0.016 \mathrm{mM}(\mathrm{ca} 20 \mu \mathrm{g} / \mathrm{mL})$, some 8-fold lower than that for NAB739. NAB739 was found to be effective in treating $E$. coli peritoneal infection in mice (dosed at $1 \mathrm{mg} / \mathrm{Kg}$ twice). ${ }^{223}$ Recently a new analog $\mathrm{NAB} 815$ has been reported, presenting $\mathrm{MIC}_{90}$ values 2 -fold less active against $E$. coli and $K$. pneumoniae and 2-fold more active against $A$. baumannii than NAB739 (both overall less active than polymyxin B). However, in vivo tests have shown that both NAB815 and NAB739 are better tolerated than polymyxin $B$ in cynomolgus monkeys. In fact, a very significant portion of the dose of NAB815 and NAB739 is excreted into urine within $8 \mathrm{~h}$ after an intravenous infusion. ${ }^{224}$ Further studies will reveal their potential use in the clinic.

Sensitizers, although sometimes devoid of intrinsic antimicrobial activity, retain the ability to permeabilize the outer membrane of Gram-negative bacteria. Polymyxin nonapeptide, a truncated version of polymyxin, is a long time kown paradigm example: it is able to increase susceptibility of $E$. coli and other Enterobacteriacea to some antibiotics, particularly lipophilic and amphiphilic ones, up to $100-$ fold times, at even low concentrations (1-3 $\mu \mathrm{g} / \mathrm{mL})$. In addition, since it lacks the N-terminal fatty tail, it is less nephrotoxic. ${ }^{221}$ NAB741 and NAB7061 are analogs of polymyxin B and NAB739, but their Nterminal lipopeptide segments are acetyl-Thr-DSer and octanoyl-ThrAbu, respectively, thus carrying only three positive charges. Both 
compounds are devoid of direct antibacterial activity but are able to potentiate the 10 - to 2000 -fold the activity of hydrophobic antibiotics (e.g. macrolides, rifampicin) or large amphiphilic antibiotics (e.g. vancomycin) against Enterobacteriaceae and $A$. baumannii.

The synergistic activity of NAB7061 has been also proven in vivo, in a mice model of $E$. coli peritoneal infection. Similarly, the combination of NAB7061 (dose $5 \mathrm{mg} / \mathrm{kg}$ ) and erythromycin $(10 \mathrm{mg} / \mathrm{kg}$ ) was also effective. ${ }^{220}$ NAB741 has recently proceeded into preclinical studies, renamed as SPR741 due to an in-license agreement with Spero Therapeutics. ${ }^{225}$ SPR741 showed low nephrotoxicity in vivo in cynomolgus monkey and rat models. Only minimal tubular degeneration was observed at a dose of $60 \mathrm{mg} / \mathrm{Kg}$.day in monkey. 226 SPR741 will enter phase I clinical trial (double-blind, placebocontrolled, ascending dose, multi-cohort trial evaluating safety, tolerability and pharmacokinetics) as a potentiator compound. ${ }^{227}$

\subsection{Cantab analogs}

Cantab Anti-Infectives has generated a series of polymyxin analogs focusing on the modification of the N-terminal fragment (Figure 10). Actually, the preparation of compounds was performed from either polymyxin nonapeptide (tetra-Boc protected) obtained from natural polymyxin $B$ by enzymatic treatment with papain or from polymyxin heptapeptide obtained from the penta-Boc protected polymyxin by treatment with savinase in acetonitrile-water mixture. ${ }^{228-231}$ Carboxylic acids to be coupled to the corresponding peptides were obtained from commercial sources or prepared in house. Some analogs (example 50 in Brown's patent) ${ }^{229}$ were also prepared by conventional Fmoc solid-phase chemistry and cyclized in solution. Amino acyl moieties in some analogs had the aminoalkyl side chain of natural Dab 1 embedded in piperazine (analog CA824) or piperidine (CA 900) rings. $\mathrm{MIC}_{90}$ of the three selected analogs CA900, CA824 and CA1049 gave similar or slightly better values than polymyxin B against typical Gram-negatives such as $E$. coli $(0.125$ vs $0.25 \mathrm{\mu g} / \mathrm{mL}$ for polymyxin B), A. baumannii $(0.25-2 \mathrm{vs} 1 \mu \mathrm{g} / \mathrm{mL}), P$. aeruginosa (1 vs $8 \mu \mathrm{g} / \mathrm{mL}$ ) and $K$. pneumoniae $(0.25$ vs $0.5 \mu \mathrm{g} / \mathrm{mL}$ ). CA900 gave particularly good activity against $A$. baumannii, with a $\mathrm{MIC}_{90}$ of $0.125 \mu \mathrm{g} / \mathrm{mL}$ compared to the one of polymyxin $(1 \mu \mathrm{g} / \mathrm{mL})$. All three analogs were less cytotoxic in HK-2 cell cultures ( $\mathrm{TC}_{50}$ of 64 , 148 and $167 \mu \mathrm{g} / \mathrm{mL}$ for CA 1049, 824 and 900, respectively) than polymyxin $\left(\mathrm{TC}_{50}\right.$ of $\left.15 \mu \mathrm{g} / \mathrm{mL}\right){ }^{232}$

In vivo efficacy was carried out with CA824 analog. In neutropenic mouse lung infection models, the antibacterial activity of CA824 proved to be superior to polymyxin B against $A$. baumannii NCTC 13301 and $P$. aeruginosa ATCC 27853 while in a mouse thigh infection model, the antibacterial activity of CA824 against the same A. baumannii strain was comparable. ${ }^{233}$ In January 2017, Spero Therapeutics acquired the assets developed by Cantab AntiInfectives to the owner Pro Bono Bio Ltd to bring the program through pre-clinical and clinical stages of development. ${ }^{234}$

\subsection{Scaffold modified polymyxins}

Throughout this review we have seen that antimicrobial potency in polymyxins tends to correlate with toxicity. Both antimicrobial activity and toxicity side effects in mammalian cells seem to be associated to the amphipathic nature of the molecule (hydrophobic and polycationic).

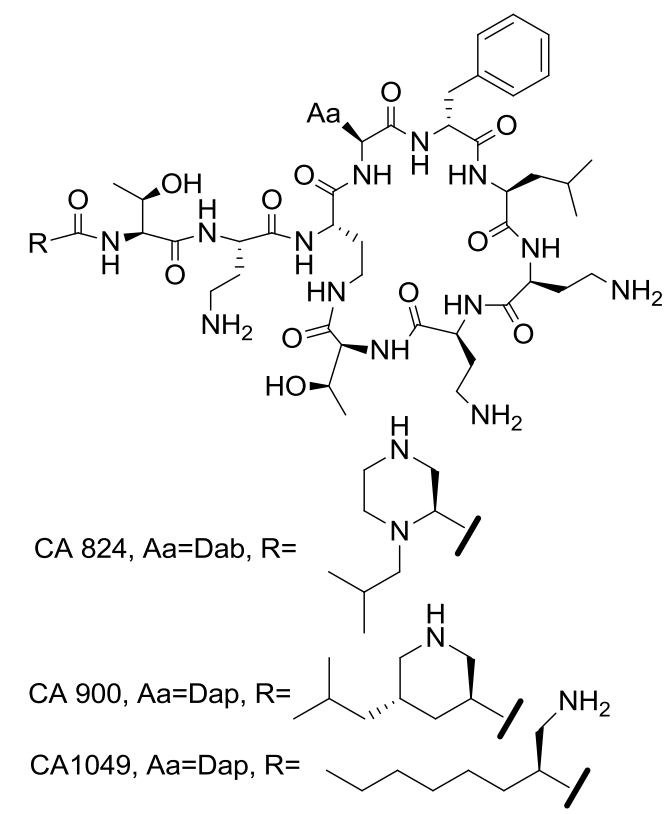

Figure 10: Chemical structures of Cantab analogs

A reasonable approach to break this parallel activity could be the modification of the backbone of the structure, a fact that is also compatible with the substitution of the amino acids of the sequence or the fatty acid to modify the periphery of the molecule. This idea would link to what it is known as soft drug design, which essentially means integrating metabolism considerations into the drug design process. ${ }^{235}$ With this purpose, different chemical units or bonds are introduced into the scaffold to help metabolism to deactivate and detoxify the molecule once it has exerted its desired antimicrobial activity.

In section 10.1., we have seen that polymyxin B and colistin are quite stable molecules. They seem not to be fully metabolized or detoxified in the kidney and accumulate in the renal cortex despite the fact that the majority of kidney's drug metabolizing and detoxification enzymes are located in the proximal tubule (see below). For instance, B- esterases, peptidases and oxidorreductases are localized relatively in high amounts in proximal tubule kidney cells.

Two examples of polymyxin analogs have been recently described in the literature that contain modifications in the peptide backbone designed to aid in the metabolization and prevent accumulation in kidneys, and hence, potentially reduce toxicity: ester-containing (i. e. depsipeptide) analogs of polymyxin (MicuRx and Barcelona) ${ }^{236,237}$ and disulfide cyclized analogs (Barcelona). ${ }^{91,237}$

\subsection{MicuRx analogs}

MicuRx has described analogs that incorporate ester, carbamate and phosphate/phosphonate/phosphoramide type of bonds within the $\mathrm{N}$-terminal part of polymyxin (Figure 11). ${ }^{236}$ The introduction of esters within polymyxin has been described before. ${ }^{229,238}$ Compounds were prepared by semisynthesis from polymyxin nonapeptide and adding conveniently protected $\mathrm{Fmoc} / \mathrm{tBu}$ amino acids, such as Boc-protected homoisoserine (L-2-hydroxy-4aminobutanoic acid), Fmoc-Dab(Boc)-OH or other acids, by means of standard coupling techniques and reagents. 


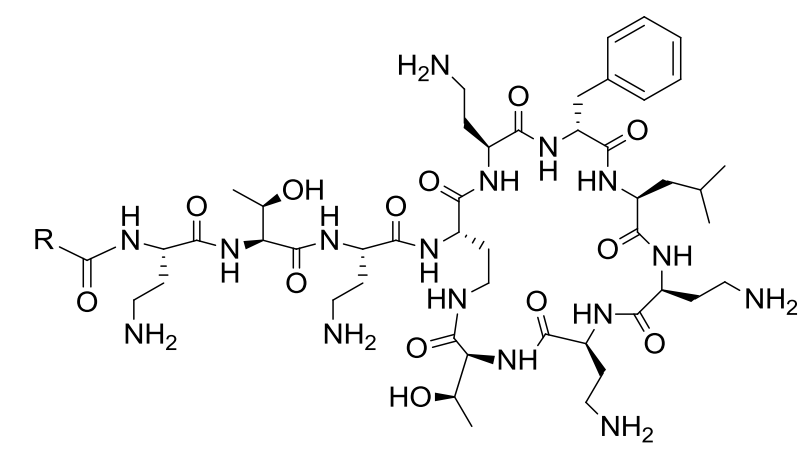

MicuRx
Example 12, $\mathrm{R}=$
MicuRx
Example 18, $\mathrm{R}=$

Figure 11: Chemical structure of MicuRx analogs and the hydrolysis products rendered by esterases

Some fifty polymyxin analogs were prepared. Analogs 12 and 18 offered antibacterial activity comparable to polymyxin B and had an ester bond embedded in the fatty acid moiety. They both yielded MIC of $2 \mu \mathrm{g} / \mathrm{mL}$ against $E$. coli (vs $2-4 \mu \mathrm{g} / \mathrm{mL}$ for polymyxin B), 2 and 4 $\mu \mathrm{g} / \mathrm{mL}$, respectively against $P$. aeruginosa (vs $2-4 \mu \mathrm{g} / \mathrm{mL}$ ) and 2 and $4 \mu \mathrm{g} / \mathrm{mL}$, respectively against $K$. pneumoniae (vs $2-4 \mu \mathrm{g} / \mathrm{mL}$ ). Cytotoxicity of both analogs in human HK-2 cells was lower $\left(\mathrm{IC}_{50}>\right.$ $200 \mu \mathrm{M})$ than that of polymyxin $\mathrm{B}\left(\mathrm{IC}_{50}=82 \mu \mathrm{M}\right){ }^{236}$

In vivo efficacy of analogs 12 and 18 in E. coli septicemia, $P$. aeruginosa thigh infection, and $P$. aeruginosa lung infection mouse models proved to be comparable to the efficacy results obtained for polymyxin B. A reduction of in vivo nephrotoxicity in rat model was observed for both in comparison with polymyxin B according to the low levels of urine biomarker NGAL (see section 11 before). Finally, stability in human plasma demonstrated that $66-67 \%$ of the original ester compounds 12 and 18 remained non-hydrolized after $1 \mathrm{~h}$ (at 4 h, $31 \%$ and $18 \%) .236$

\subsection{Barcelona analogs}

The University of Barcelona has described analogs of polymyxin where the amide bond between the $\mathrm{\gamma}$-amino group of Dab4 and the C-terminal carboxy group of Thr10 has been replaced with a disulfide bond (Figure 12). ${ }^{91}$ This replacement is isosteric and implies the substituition of those amino acids with cysteines with an appropriate configuration of the $\alpha$-carbon (L-cysteine in position 4 and D-cysteine in 10). The disulfide link may provide polymyxin analogs with sufficient stability to reach the infectious target in vivo. However, in an eventual accumulation and uptake by renal cells, the disulfide bond may be broken thus opening up the cyclic heptapeptide due to the reducing intracellular environment (reduced glutathione and oxidorreductases) that could facilitate peptide proteolysis and potentially lower renal toxicity. In this respect, detoxification of xenobiotics in kidney is carried out by metabolizing enzymes that are involved, for instance, in the conjugation of glutathione, glucuronic acid, or sulfate. These enzymes, including cytochrome P-450, are mainly localized in kidney's proximal tubules.

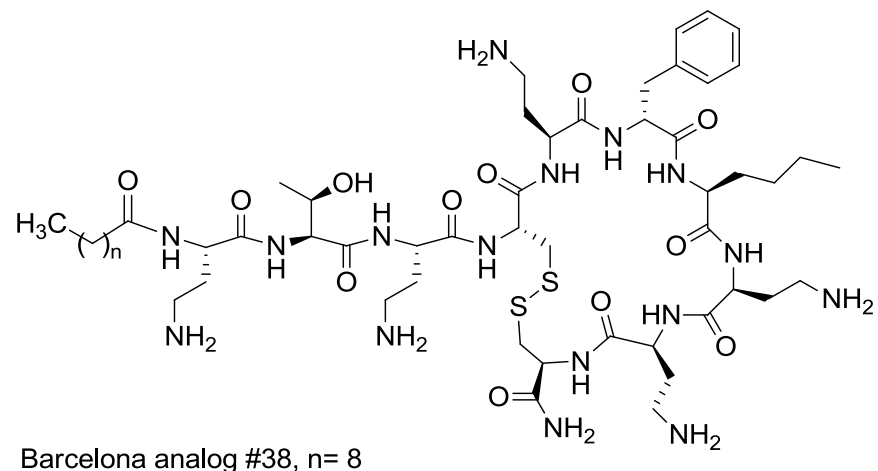

Barcelona analog \#39, $n=10$

Figure 12: Chemical structure of Barcelona disulfide analogs

In particular, proximal tubular cells exhibit a high activity in glutathione redox cycle enzymes, such as glutathione disulfide reductase, GSH peroxidases, and GSH S-transferase. The intracellular concentration of glutathione in proximal tubular cells is around 2-5 $\mathrm{mM}$ whereas in the lumen is at least two orders of magnitude lower. In blood, GSH concentration is around $20 \mu \mathrm{M} .{ }^{203,239,240}$

Furthermore, in an in vivo study in rat dealing with the metabolism and retention of octreotide (a marketed disulfide cyclic peptide also containing two D-amino acids) ${ }^{91}$ in kidney and liver, metabolites of radiolabeled octreotide were shown to be decyclized (linear) products by reduction of the disulfide bond to cysteine and fragmented by hydrolysis of the peptide bonds. ${ }^{241}$

All this data supports the use of the disulfide bond as a tool to modulate the stability of the polymyxin scaffold, facilitate metabolization and reduce accumulation in kidney. In fact, the use of disulfide bonds in drugs should not be seen as exceptional. There are several peptide drugs in the market containing disulfide bonds, such as octreotide, lanreotide or vapreotide (analogs of somatostatin that consist of a disulfide cyclic octapeptide), insulin, linaclotide (for inflammatory bowel syndrome), ziconotide (treatment of pain), pramlintide (for type II diabetes), atosiban (inhibitor of the hormones oxytocin and vasopressin), neseritide (for acute decompensated congestive heart failure), romidepsin (anticancer agent used in cutaneous T-cell lymphoma), and peginesatide (treatment of anemia associated with chronic kidney disease).

Preparation of the analogs was carried out by $\mathrm{Fmoc} / \mathrm{tBu}$ solid phase chemical synthesis. Cyclization by means of disulfide bond formation was performed at high dilution conditions in plain water and dimethylsulfoxide solvent, with no other reagents. Antibacterial activity comparable to polymyxin B was achieved, including resistant and multi-drug resistant strains. Analogs 38 and 39 yielded both MIC of 2 and $4 \mu \mathrm{g} / \mathrm{mL}$, respectively against $E$. coli (vs $1 \mu \mathrm{g} / \mathrm{mL}$ for polymyxin B), $1 \mu \mathrm{g} / \mathrm{mL}$ against $P$. aeruginosa (vs $2 \mu \mathrm{g} / \mathrm{mL}$, polymyxin B). Against resistant strains, Analogs 38 and 39 yielded both MIC of 0.5 and $0.5-1 \mu \mathrm{g} / \mathrm{mL}$, respectively against $E$. coli (vs $0.25-0.5 \mu \mathrm{g} / \mathrm{mL}$ for polymyxin $B$ ) and $0.5-4 \mu \mathrm{g} / \mathrm{mL}$ against $P$. aeruginosa (vs $1-2$ $\mu \mathrm{g} / \mathrm{mL}$, polymyxin B)..$^{91}$ Whatsmore, analog 39 was also active against Gram-positive bacteria S. aureus and Enterococcus faecalis, rendering comparable MIC to those of daptomycin and vancomycin. 
Analog 38 has also demonstrated synergistic and antibiofilm activities with imipenem in a imipenen-resistant strain of $P$. aeruginosa. ${ }^{242}$ Finally, an in vivo acute toxicity test by subcutaneous administration performed on CD-1 mice with analog 38, the LD50 obtained $(283 \mathrm{mg} / \mathrm{kg})$ was clearly superior to the one reported for polymyxin $B(59.5 \mathrm{mg} / \mathrm{kg}) .^{91}$ Further in vivo tests (pharmacokinetic studies, full toxicity and efficacy) are ongoing and will be reported in due course.

\section{Conclusions and Perspectives}

The emergence of multi-drug resistant Gram-negative bacteria will need of all efforts (antimicrobial chemical design, medicinal chemistry, microbiology, pharmacology, development, clinical studies, rational use of antibiotics) to control this new health challenge.

The research and development of alternative antimicrobial agents is a plausible option to tackle the resistance problem and the "antibiotic discovery void" since the late 1980s. Unfortunately, in the field of polymyxins, only a single candidate, CB-182,804 has progressed to a clinical phase but was finally discontinued. Recently, a new polymyxin molecule, SPR741, has been announced to proceed to clinical phases albeit as a potentiator, not as an antimicrobial per $s e .{ }^{227}$ Hence, much effort and new approaches are still needed.

The design, discovery and development of new chemical entities, particularly those with new scaffolds or acting by new mechanism of action will certainly play a fundamental role. Furthermore, advances in the understanding at the molecular level of the mechanism of polymyxin toxicity will provide new insights on how to design new molecules with better therapeutic indexes. In this sense, the incorporation of metabolism considerations into the drug design process is a promising new line. New challenges will rise, though, as these new chemical units need to keep a balance between stability to reach the target and exert the desired antimicrobial activity in vivo and later facilitate metabolization and detoxification of the molecule thus preventing unwanted side effects.

In the short run, natural polymyxin $\mathrm{B} /$ colistin and colistimethate will continue to be used as last-line therapeutic option due to the scarcity of antibiotics against MDR Gram-negative bacteria. Nevertheless, recent pharmacological progress and understanding of the mechanism of action and toxicity in polymyxins provides clinicians with valuable information for optimizing their use in patients (dosage, combinations, nephrotoxicity, biomarkers). ${ }^{243}$ Still a large amount of work is necessary for instance to clarify the advantage of antibiotic combinations with polymyxins. In this sense, clinical trials to compare colistin monotherapy and the combination with meropenem are in progress. ${ }^{150}$

We hope that the next generation of polymyxins will become satisfactory therapeutic tools for the treatment of infections caused by multi-drug resistant bacteria.

\section{Acknowledgements}

We thank the support of the University of Barcelona, Fundació Bosch i Gimpera, Xarxa de Referència en Biotecnologia (XRB, Generalitat de Catalunya) and the European Institute of Innovation and Technology (EIT Health). The authors are members of the ENABLE (European
Gram-Negative Antibacterial Engine) consortium (IMI-ND4BB, http://www.imi.europa.eu/content/enable).

\section{References}

1 R. G. Benedict and A. F. Langlykke, J. Bact. 1947, 54, 24

2 P. G. Stansly, R. G. Shepherd and H. J. White, Johns Hopk. Hosp. Bull. 1947, 81, 43

3 G. C. Ainsworth, A. M. Brown and G. Brownlee, Nature, 1947, 160, 263

4 J. A. Orwa, C. Govaerts, R. Busson, E. Roets, A. Van Schepdael and J. Hoogmartens, J. Chromatogr. A, 2001, 912, 369.

5 D. Decolin, P. Leroy, A. Nicolas and P. Archimbault, J Chromatogr Sci., 1997, 35, 557

6 J. A. Orwa, C. Govaerts, R. Busson, E. Roets, A. Van Schepdael and J. Hoogmartens, J Antibiot (Tokyo), 2001, 54, 595

7 J. He, S. Gao, M. Hu, D. S.-L. Chow and V. H. Tam, J Antimicrob. Chemother. 2013, 68, 1104

8 M. E. Falagas, K. N. Fragoulis, , S. K. Kasiakou, G. J. Sermaidis, and, A. Michalopoulos, Int. J. Antimicrob. Agents 2005, 26, 504

9 D. N. Gilbert, R.J. Guidos, H. W. Boucher, G.H. Talbot, B. Spellberg, J. E. Edwards Jr, W. M. Scheld, J. S. Bradley, J. G. Bartlett, Clin. Infect. Dis. 2010, 50, 1081

10 CDC webpage: https://www.cdc.gov/drugresistance/about.html

11 F. Rabanal and Y. Cajal, In: New Weapons to Control Bacterial Growth, pp. 433-452, Springer International Publishing Switzerland 2016, T.G. Villa and M. Viñas (eds.)

12 J. M Sierra, E. Fusté, F. Rabanal, T. Vinuesa and M. Viñas, Expert Opinion on Biological Therapy, 2017, 6, 663-76

13 WHO press release: http://www.who.int/mediacentre/news/releases/2017/bact eria-antibiotics-needed/en/

14 T. Velkov, P. E. Thompson, R. L. Nation and J. Li, J. Med. Chem. 2010, 53, 1898.

15 T. Velkov, K. D.Roberts, R. L. Nation, P. E. Thompson, J. Li, Future Microbiol. 2013, 8, 711

16 M. Vaara, J. Antimicrob. Chemother. 2013, 68, 1213

17 P. Brown and M. J. Dawson, J. Antibiot., 2017, 70, 386

18 W. Raza, W. Yang and Q-R. Shen, Journal of Plant Pathology 2008, 90,419

19 G. Brownlee, S. R. M. Bushby and E. I. Short Brit. J. Pharmacol. $1952,7,170$

20 Y. Koyama, A. Kurosawa, A. Tuchiya and K. Takahashi, J. Antibiotics (Tokyo) Ser.B., 1950, 3, 457

$21 \mathrm{~K}$. Hayashi and T. Suzuki, Kyoto University Research information Repository, 1965, http://repository.kulib.kyotou.ac.jp/dspace/bitstream/2433/76067/1/chd043_3_259.pdf

22 D. R. Storm, K. S. Rosenthal and P. E Swanson Ann. Rev. Biochem. 1977, 46, 723

23 N. I. Martin, H. Hu, M. M. Moake, J. J. Churey, R. Whittal, R. W. Worobo and J. C. Vederas, J. Biol. Chem. 2003, 278, 13124

24 B. Niu, J. Vater, C. Rueckert, J. Blom, M. Lehmann, J.-J. Ru, X.H. Chen, Q. Wang and R. Borriss BMC Microbiology, 2013, 13, 137

25 Y. Kimura, E. Murai, M. Fujisawa, T. Tatsuki and F. Nobue, J Antibiot. 1969, 22, 449

26 J. Shoji, H. Hinoo, Y. Wakisaka, K. Koizumi, M. Mayam and S. Matsuura J. Antibiot., 1977, 30, 1029

27 J. Shoji, T. Kato, H. Hinoo, 1977. J. Antibiot., 1977, 30, 1035

28 J. Shoji, T. Kato, H. Hinoo, 1977. J. Antibiot., 1977, 30, 1042

29 J. Shoji, T. Kato, H. Hinoo, 1977. J. Antibiot., 1977, 30, 427

30 W. L. Parker, M. L. Rathnum, L. D. Dean, M. W. Nimeck, W. E. Brown and E. Meyers, J. Antibiot. 1977, 30, 767

31 S. Terabe, R. Konaka and J. Shoji. J. Chromat. 1979, 173, 313

32 S. A. Cochrane and J. C. Vederas, Medicinal Res. Rev., 2016, 36, 4 
33 M. Shaheen, J. Li, A. C. Ross, J. C. Vederas, and S.E. Jensen, Chem. Biol. 2011, 18, 1640

34 T. Velkov, K. D. Roberts and J. Li, Nat. Prod. Rep., 2017,34, 295

35 M.E. Falagas and S.K. Kasiakou, Clin. Infect. Dis., 2005, 40, 1333

36 T. Mogi, Y. Murase, M. Mori, K. Shiomi, S. Omura, M.P. Paranagama and K. Kita, J. Biochem. 2009, 146, 491-499.

37 N. Rastogi, M. C. Potar AND H. L. David, Ann Inst Pasteur Microbiol. 1986, 137, 45

38 B. Zhai and X. Lin, Int. J. Antimicrob Agents, 2013;41:250-254

39 J. He, K. R. Ledesma, D. A. Figueroa, T.-P. Lim, D. S. Chow and V. H. Tam. Int. J. Antimicrobial Agents, 2010, 35, 308

40 V. H. Tam, H. Cao, K. R. Ledesma and M. Hu, Antimicrob. Agents Chemother. 2011, 55,4490

41 K. D. Roberts, M. A. K. Azad, J. Wang, A. S. Horne, P. E. Thompson, R. L. Nation, T. Velkov and J. Li, ACS Infect. Dis. 2015, 1, 568

42 Z. Kassamali, R. A. Prince, L. H. Danziger, J. C. Rotschafer, P. R. Rhomberg and R. N. Jones Antimicrob. Agents Chemother. 2015, 59, 7823

43 P. Manchandani, Y. Dubrovskaya, S. Gao and V. H. Tam Antimicrob. Agents Chemother. 2016, 60, 6980

44 S. Sivanesan, K .Roberts, J. Wang, S.-E. Chea, P. E. Thompson, J. Li, R. L. Nation, and T. Velkov J. Nat. Prod., 2017, 80, 225

$45 \mathrm{~A}$. Abdelraouf, J. He, K. R. Ledesma, M. Hu and V. H. Tam, Antimicrob Agents Chemother , 2012, 56, 5724

46 M. E. Falagas, K. Z. Vardakas, http://www.antimicrobe.org/d05.asp

47 M. Barnett, S. R. Bushby and S. Wilkinson, Br. J. Pharmacol. Chemother., 1964,23, 552

48 J. Li, R.W. Milne, R.L. Nation, J.D. Turnidge, T.C. Smeaton and K. Coulthard, J. Antimicrob. Chemother., 2004, 53, 837

49 Bergen, P.J.; Li, J.; Rayner, C.R.; Nation, R.L. Colistin methanesulfonate is an inactive prodrug of colistin against Pseudomonas aeruginosa. Antimicrob. Agents Chemother., 2006, 50, 1953-1958.

50 J. Li, R.W. Milne, R.L. Nation, J.D. Turnidge, and K. Coulthard, Antimicrob. Agents Chemother., 2003, 47, 1364

51 S. Marchand, I. Lamarche, P. Gobin and W. Couet, J Antimicrob Chemother, 2010, 65, 1753

52 R. L. Nation, T. Velkov and J. Li, Clin. Infect. Dis., 2014, 59, 88

53 S. Biswas, J.M. Brunel, J.C. Dubus, M. Reynaud-Gaubert and J.M. Rolain, Expert Rev. Anti.Infect.Ther. 2012, 10, 917

54 European Medicines Agency review of polymyxin-based medicines:

http://www.ema.europa.eu/ema/index.jsp?curl=pages/news and events/news/2014/10/news detail 002194.jsp\&mid= WC0b01ac058004d5c1

55 H.I. Zgurskaya, C.A. López and S. Gnanakaran, ACS Infect. Dis., 2015, 1, 512-522.

56 H. Nikaido, Microbiol. Mol. Biol. Rev., 2003, 67, 593-656.

57 R.M. Epand and R.F. Epand, Biochim. Biophys. Acta, 2009, 1788, 289-294.

58 K. Nishino, F.F. Hsu, J. Turk, M.J. Cromie, M.M. Wösten and E.A. Groisman, Molecular Microbiology, 2006, 61, 645-654.

59 C.R.H. Raetz and C. Whitfield, Annual Review of Biochemistry, 2002, 71, 635-700.

60 M. Schindler and M.J. Osborn, Biochemistry, 1979, 18, 44254430.

61 M.E. Evans, D.J. Feola and R.P. Rapp, The Annals of Pharmacotherapy, 1999, 33, 960-967.

62 R.E. Hancock and D.S. Chapple, Antimicrob. Agents Chemother., 1999, 43, 1317-1323.

63 R.E. Hancock, J Med. Microbiol., 1997, 46, 1-3.

64 R.E. Hancock and R. Lehrer, Trends Biotechnol., 1998, 16, 8288.

65 M. Vaara and T. Vaara, Nature, 1983, 303, 526-528.
66 S.E. Cheah, M.D. Johnson, Y. Zhu, B.T. Tsuji, A. Forrest, J.B. Bulitta, J.D. Boyce, R.L. Nation and J. Li, Sci. Reports, 2016, 6 , 26233.

67 M.D. Bruch, Y. Cajal, J.T. Koh and M.K. Jain, J. Am. Chem. Soc., 1999, 121, 11993-12004.

68 P. Pristovsek and J. Kidric, J. Med. Chem. 1999, 42, 4604-4613.

69 J.J. Meredith, A. Dufour and M.D. Bruch, J. Phys. Chem. B, 2009, 113, 544-551.

70 S. Bhattacharjya, V.I. Mathan and P. Balaram, Biopolymers, 1997, 41, 251-265.

71 J.T. Oh, Y. Cajal, P.S. Dhurjati, T.K. Van Dyk and M.K. Jain, Biochim Biophys Acta, 1998, 1415, 235-245.

72 L. Zhang, P. Dhillon, H. Yan, S. Farmer and R.E.W. Hancock, Antimicrob. Agents Chemother., 2000, 44, 3317-3321.

73 N.P. Mortensen, J.D. Fowlkes, C.J. Sullivan, D.P. Allison, N.B. Larsen, S. Molin and M.J. Doktycz, Langmuir, 2009, 25, 3728 3733.

74 R. Daugelavicius, E. Bakiene and D.H. Bamford, Antimicrob. Agents Chemother., 2000, 44, 2969-2978.

75 K.A. Brogden, Nat. Rev Microbiol, 2005, 3, 238-250.

76 Z. Yu, W. Qin, J. Lin, S. Fang and J. Qiu, BiomedRes.Int. 2015, $1-11$.

77 J.T. Oh, T.K. Van Dyk, Y. Cajal, P.S. Dhurjati, M. Sasser and M.K. Jain, Biochem. Biophys. Res. Commun., 1998, 246, 619-623.

78 M.M. Domingues, M.L. Bianconi, L.R.S. Barbosa, P.S. Santiago, M. Tabak, M.A.R.B. Castanho, R. Itri and N.C. Santos, Biochim. Biophys. Acta, 2013, 1828, 2419-2427.

79 J.T. Oh, Y. Cajal, E.M. Skowronska, S. Belkin, J. Chen, T.K. Van Dyk, M. Sasser and M.K. Jain, Biochim. Biophys. Acta, 2000 1463, 43-54.

80 Y. Cajal, J. Rogers, O. Berg and M.K. Jain, Biochemistry, 1996, 35, 299-308

81 Y. Cajal, J. Ghanta, K. Easwaran, A. Surolia and M.K. Jain, Biochemistry, 1996, 35, 5684-5695

82 A. Clausell, M.A. Busquets, M. Pujol, M.A. Alsina and Y. Cajal, Biopolymers, 2004, 75, 480-490.

83 H.H. Yim and M. Villarejo, J. Bacteriol., 1992, 174, 3637-3644

84 A. Liechty, J. Chen and M.K. Jain, Biochim Biophys Acta, 2000 1463, 55-64.

85 R. Henry, B. Crane, D. Powell, L.D. Deveson, Z. Li, J. Aranda, P. Harrison, R.L. Nation, B. Adler, M. Harper, J.D. Boyce and J. Li, J. Antimicrob. Chemother., 2015, 70, 1303-1313.

86 A. Clausell, F. Rabanal, M. Garcia-Subirats, M.A. Alsina and Y Cajal, J. Phys. Chem. B, 2006, 110, 4465- 4471.

87 A. Clausell, F. Rabanal, M. Garcia-Subirats, M. A. Alsina and Y. Cajal, Luminescence, 2005, 20, 117-123.

88 A. Clausell, M. Garcia-Subirats, M. Pujol, M.A. Busquets, F. Rabanal and Y. Cajal, J. Phys. Chem. B, 2007, 111, 551-563.

89 A. Grau-Campistany, A. Manresa, M. Pujol, F. Rabanal and Y. Cajal, Biochem. Biophys. Acta, 2016, 1858, 333-343.

90 A. Grau-Campistany, M.Pujol, A.M. Marqués, A. Manresa, F. Rabanal and Y. Cajal, Colloids and Surfaces A, 2015, 480, 307317.

91 F. Rabanal, A. Grau-Campistany, X. Vila-Farrés, J. GonzalezLinares, M. Borràs, J. Vila, A. Manresa and Y. Cajal. Sci. Rep. 2015, 5, 1-11.

92 Z. Z. Deris ZZ, J. Akter, S. Sivanesan, K.D. Roberts, P.E. Thompson, R.L. Nation, J. Li and T. Velkov, The Journal of Antibiotics, 2014, 67, 147-151.

93 J.N. Blaza, H.R. Bridges, D. Aragão, E.A. Dunn, A. Heikal, G.M. Cook, Y. Nakatani and J. Hirst, Sci. Rep., 2017, 7, 40165-40176.

94 D.C. LaPorte, K.S. Rosenthal and D.S. Storm, Biochemistry, 1977, 16, 1642-1648.

95 G.H. De Zoysa and V. Sarojini, ACS Appl. Mater. Interfaces, 2017, 9, 1373-1383.

96 M. Rhouma, F. Beaudry, W. Thériault and A. Letellier, Front. Microbiol., 2016, 7, 1789. 
97 Z.Z. Deris, J.D. Swarbrick, K.D. Roberts, M.A. Azad, J. Akter A.S. Horne, R.L. Nation, K.L. Rogers, P.E. Thompson, T. Velkov and J. Li, Bioconjug. Chem., 2014, 25, 750-760.

98 T.R. Sampson, X. , M.R. Schroeder, C.S. Kraft, E.M. Burd and D.S. Weiss, Antimicrob. Agents Chemother., 2012, 56, 5642-5649.

99 P.I. Pereira Ramos, M.G. Flores Custódio, G. Quispe Saji, T. Cardoso, G. Lucchetti da Silva, G. Braun, W.M.B.S. Martins, R. Girardello, A.T. Ribeiro de Vasconcelos, E. Fernández, A.C. Gales and M.F. Nicolás, BMC Genomics, 2016, 17, 447-462.

100 J.A. Imlay, Nat. Rev. Microbiol., 2013, 11, 443-454.

101 S. Pournaras, A. Poulou, K. Dafopoulou, Y.N. Chabane, I. Kristo, D. Makris, J. Hardouin, P. Cosette, A. Tsakris and E. Dé, Antimicrob Agents Chemother.,2014, 58, 828-32.

102 A. O. Olaitan, S. Morand and J. M. Rolain, Frontiers in Microbiology 2014, 5, 643.

103 J. M. A. Blair, M. A. Webber, A. J. Baylay, D. O. Ogbolu and L. J. V. Piddock. Nature Microbiology, 2015, 13, 43

104 L. Poirel, A. Jayol, and P. Nordmann. Clin. Microbiol. Rev. 2017, 30, 557-596

105 K. Jeannot, A. Bolard, and P. Plésiat Int. J. Antimicrobial Agents 2017, 49, 526-535

106 M. Vaara, T. Vaara, M. Jensen, I. Helander, M. Nurminen, E. Th. Rietschel, P.H. Mäkelä, FEBS Lett., 1981, 129, 145-149

107 Y.-Y. Liu, Y. Wang, t. M. Walsh; L-X. Yi et al. Lancet Infectious Diseases, 2016, 16, 161-168

108 A. P Magiorakos, A. Srinivasan, R. B. Carey, Y. Carmeli, M. E. Falagas, C. G. Giske, et al. Clin Microbiol Infect. 2012, 18, 268-81.

109 E. K. Schneider, F. Reyes-Ortega, T. Velkov and J. Li Essays in Biochem. 2017, 61 115-125

110 M. F. Richter, B. S. Drown, A. P. Riley, A. Garcia, T. Shirai, R. L. Svec and P. J. Hergenrother, Nature, 2017, published online 10 May 2017, doi:10.1038/nature22308

111 O. Zusman, t, Avni, I. Leibovici, A. Adler, I. Friberg, t. Stergiopoulou T, Y. Carmeli, P. Mical Antimicrob. Agents Chemother., 2013, 57, 5104-5111

112 J. He, k. Kaniga, A. S. Lynch, R. K. Flamm, T. A. , Diagn. Microbiol. Infect. Dis., 2012, 74, 417-419

113 B. W. Gunderson, K. H. Ibrahim, L. B. Hovde, T. L. Fromm, M. D. Reed and J. C. Rotschafer, Antimicrob. Agents Chemother. 2003, 47, 905-9

114 B. B. D'Souza, S. R. Padmaraj, P. D. Rekha, R. C. Tellis, S. Prabhu and P. Pothen, Microb. Drug Resist, 2014, 20, 550-4

115 G. Samonis, S. Maraki, D. E. Karageorgopoulos, E. K. Vouloumanou and M. E. Falagas ME. Eur. J. Clin. Microbiol. Infect. Dis., 2012, 31, 695-701

116 G. A. Pankuch, H. Seifer, P. C. and Appelbaum., Diagn. Microbiol. Infect. Dis., 2010 , 67, 191-7

117 X Di, R. Wang, B. Liu, Z. Xin, N. Wentao, W. Jin, L Beibei, C. Yun and L. Youning, J. Antibiot. (Tokyo), 2015, 68, 551-555

118 M. Berditsch, T. Jäger, N. Strempel, T. Schwartz, J. Overhage, A. S. Ulrich, Antimicrob Agents Chemother. 2015, 59, 5288.

119 M. Berditsch, H.Lux, O. Babii, S. Afonin and A. S. Ulrich Pharmaceuticals 2016, 9, 56

120 C. Tascini, G. Gemignani, S. Ferranti, E. Tagliaferri, A. Leonildi, A. Lucarini and F. Menichetti, J Chemother, 2004, 16, 282-287

121 R. Sharma, S. Patel, C. Abboud, J. Diep, N. S. Ly, J. M. Pogue K. S. Kaye. J. Li, G. G. Rao, Int. J. Antimicrobial Agents, 2017, 49, 224-232.

122 M. Souli, I. Galani, S. Boukovalas, m. G. Gourgoulis , Z. Chryssouli, K. Kanellakopoulou, T. Panagea and H. Giamarellou, Antimicrob. Agents Chemother., 2011, 55, 2395-7

123 M. S. Albur, A. Noel, K. Bowker and A. McGowan Int. J. Antimicrobial Agents, 2015, 46, 560-567
124 C. Vidaillac, L. Benichou and R. E. Duval, , Antimicrob. Agents Chemother., 2012, 56, 4856-61.

125 G. A. Pankey and D. S. Ashcraft, Diagn. Microbiol. Infect. Dis., 2011, 70, 561-4.

126 P. Galbani, D. Lombardo, R. E. Lewis, M. Mercuri, S. Bonora, M. P. Landini and S. Ambretti, J. Antimicrob. Chemother., 2014, 69, 1856-65

127 N. Abdul Rahim, S. E. Cheah, M. D. Johnson, H. Yu, h. E. Sidjabat, J. Boyce, et al. J. Antimicrob. Chemother., 2015, 70, 2589

128 I. Rodríguez-Avial, I. Pena, J. J. Picazo, C. Rodríguez-Avial and E. Culebras. Int.J. Antimicrobial Agents, 2015, 46, 616-21

129 T. Tängdén, R. A. Hickman, P. Forsberg, P. Lagerbäck, C. G. Giske and O. Cars, Antimicrobial Agents Chemother., 2014, 58, 1757-1762.

130 M. Bassetti, E. Repetto, E. Righi et al. J. Antimicrob. Chemother., 2008, 61,417-420

131 S. Motaouakkil, B. Charra, A. Hachimi, H. Nejmi, A. Benslama, N. Elmdaghri, H. Belabbes and M.Benbachir M. J. Infect., 2006, 53, 274-278

132 G. M. Hogg, J. G. Barr and C. H. Webb. J. Antimicrob. Chemother., 1998, 41, 494-495

133 H. J. Lee, P. J. Bergen, J. B. Bulitta, B. Tsuji, A. Forrest, R. L. Nation, et al. Antimicrob Agents Chemother. 2013, 57, 3738-45.

134 N. Petrosillo, E. Ioannidou and M. E. Falagas. Clin. Microbiol.Infect., 2008, 14, 816-827

135 D. W. Wareham, N. C. Gordon and M. Hornsey., J Antimicrob. Chemother. 2011, 66, 1047-51.

136 N. C. Gordon, K. Png and D. W. Wareham, Antimicrob. Agents Chemother. 2010, 54, 5316-22.

137 M. Hornsey and D. W. Wareham, Antimicrob. Agents Chemother. 2011, 55, 3534-7

138 M. Hornsey, C. Longshaw, L. Phee and D. W. Wareham, Antimicrob. Agents Chemother. 2012, 56, 3080-5.

139 M. Hornsey, L. Phee, C. Longshaw and D. W. Wareham, Int J Antimicrob Agents, 2013, 41, 285-7.

140 W. Ni, X. Shao, X. Di, J. Cuiand R. Y. Wang, Int. J. Antimicrob. Agents. 2015, 45, 8-18

141 B. Fan, J. Guan, X. Wang and Y. Cong, PLoS ONE. 2016, 11, e0157757

142 F. Timurkaynak, F. Can, O. K. Azap, M. Demirbilek, H. Arslan and S. O. Karaman. Int. J. Antimicrob. Agents, 2006, 27, 224-228

143 C. H. Lee, Y. F. Tang YF, L. H. Su LH, C. C. Chien, CC, J. W. Liu, Microb. Drug Resis.t, 2008, 14, 233-237

144 D. R. Bowers, H. Cao, J. Zhou, K. R. Ledesma, D. Sun, O. Lomovskaya, et al. Antimicrob Agents Chemother. 2015, 59, 2720-5.

145 H. Yang, G. Chen, L. Hu, Y. Liu,, J. Cheng, H. Li , Y. Ye and J. Li, Int. J. Antimicrob. Agents, 2015, 45, 188-191

146 D. Field, N. Seisling, P. D. Cotter, R. P. Ross, C. Hill, Frontiers in Microbiol.. 2016, 7, 1713.

147 W. Liang, X. .F Liu, J. Huang, D. M. Zhu, j. Li and J. Zhang, BMC Infect. Dis. 12011, 11, 109

148 J. R. Lenhard, R. L. Nation, and B. T. Tsuji, 2016, 48, 607-613.

149 X. Zhang, F. Guo, H. Shao, X. Zheng, Journal of Infection 2017, 74, 118-130

150 Trial for the Treatment of Extensively Drug-Resistant Gram-negative https://clinicaltrials.gov/ct2/show/NCT01597973 https://clinicaltrials.gov/ct2/show/NCT01732250

151 S. N. Schwartz, G. Medoff, G. S. Kobayashi, C. N. Kwan, and D. Schlessinger Antimicrob. Agents Chemother., 1972, 2, 3640

152 B. Zhai , H. Zhou, L. Yang, J. Zhang, K. Jung, C.-Z. Giam, X. Xiang and X. Lin J. Antimicrob. Chemother., 2010, 65, 931-938 153 N.A. Moneib, J. Chemother., 1995, 7, 525-529. 
154 S. Pietschmann, K. Hoffmann, M. Voget and U. Pison. Veterinary Res. Comm.. 2009, 33, 489-505.

155 R. Ben-Ami, R. E. Lewis, J. Tarrand, K. Leventakos and D. P. Kontoyiannis, Antimicrob. Agents Chemother. 2010, 54, 484490.

156 H. Schemuth, S. Dittmer, S., M. Lackner, L. Sedlacek, A Hamprecht, E. Steinmann, J. Buer, P. M. Rath, J. Steinmann, Mycoses, 2013, 56, 297-303.

157 T. P. Venturini, L. Rossato, F. Chassot , J. Tairine Keller, F. Baldissera Piasentin, J. Morais Santurio, S. Hartz Alves, J. Med. Microbiol, .2016, 65, 770-4.

158 L-H Hsu, H-F Wang, P-L Sun, F-R Hu, and Y-L Chen_Int. J. Antimicrobial Agents, 2017, in press

159 E. K. Adams, D. S. Ashcraft and G. A. Pankey, Am. J. Med. Sci. 2016, 35, 265-70.

160 G. Pankey, D. Ashcraft, H. Kahn and A. Ismail, Antimicrob. .Agents Chemother. 2014, 58, 5795-5800

161 U. Zeidler, M.E. Bougnoux, A. Lupan, A. et al. J Antimicrob Chemother. 2013, 68, 1285-1296

162 S. M. Ribeiro, M. R. Felício, E. Vilas Boas, S. Gonçalves , F. F. Costa , R. Perumal Samy, N. C. Santos and O. L. Franco, Pharmacology \& Therapeutics , 2016,160 133-144

163 R. Gopal, Y. G. Kim, J. H. Lee, S. K. Lee, J. D. Chae, B. K. Son , C. H. Seo and Y. Park, Antimicrob. Agents Chemother., 2014, 58, $1622-1629$

164 J. Lora-Tamayo, O. Murillo, P.J. Bergen, R.L. Nation, A. Poudyal, X. Luo, H.Y. Yu, J. Ariza, and J. Li, J. Antimicrob. Chemother. 2014, 69, 2434-2442

165 C. de la Fuente-Núñez, F. Reffuveille, E. F. Haney, S. K. Straus and R. E. Hancock. PLoS Pathog. 2014,10, e1004152

166 K. Tarquinio, K. Confreda, J. Shurko and K. LaPlante, Antimicrobial Agents and Chemotherapy. 2014, 58, 17231729.

167 R.Rosales-Reyes, M.D. Alcántara-Curiel, M.D. JarilloQuijada, C. Gayosso-Vázquez, M. Morfin-Otero, E. RodríguezNoriega and J. Santos-Preciado, Chemotherapy, 2015-2016, 61, 8-14

168 I. d'Angelo, B. Casciaro, A. Miro, F Quaglia, M. L. Mangoni and F. Ungaro, Colloids Surf. B 2015, 135, 717-725.

169 C. de la Fuente-Núñez, M. H. Cardoso, C. E. de Souza, O. L., Franco and R. E. W. Hancock, Biochim. Biophys. Acta, 2016, 1858, 1061-1069

170 W. Wei, H. Yang, L. Hu, Y. Ye and J. Li - Journal of Microbiology, Immunology and Infection, 2015, xx, 1e10, [Epub ahead of print]

171 D. S. Akajagbor, S. L. Wilson, K. D. Shere-Wolfe, P. Dakum, M. E. Charurat and B. L. Gilliam, Clin Infect Dis. 2013, 57, 13003.

172 M. E. Falagas and S. K. Kasiakou Critical Care. 2006, 10(1):R27. doi:10.1186/cc3995.

173 M. E. Falagas, P. I. Rafailidis, E. loannidou, V. G. Alexiou, D. K. Matthaiou, D. E. Karageorgopoulos, A. Kapaskelis, D. Nikita, A. Michalopoulos, Int. J. Antimicrob. Agents 2010, 35, 194199

174 J. A. Kwon, J. E. Lee, W. Huh, K. R. Peck, Y. G. Kim, D. J. Kim, H. Y. Oh. Int. J. Antimicrob. Agents 2010, 35, 473-477

175 J. D. Hartzell, R. Neff, J. Ake, R. Howard, S. Olson, K. Paolino , M. Vishnepolsky, A. Weintrob and G. Wortmann, Clin. Infect. Dis. 2009, 48, 1724-1728

176 H. Ko, M. Jeon, E. Choo, E. Lee, T. Kim, J. B. Jun and H. W. Gil, Nephron. Clin. Pract. 2011, 117, c284-c288

177 A. Trifi, S. Abdellatif, F. Daly, K. Mahjoub, R. Nasri, M Oueslati, R. Mannai, M. Bouzidi and S. Ben Lakhal, Chemotherapy, 2016, 61, 190-6.

178 C. A. DeRyke, A. J. Crawford, N. Uddinand M. R. Wallace, Antimicrob. Agents Chemother., 2010, 54, 4503-4505
179 C. Dai, S. Tang, S. Deng, S. Zhang, Y. Zhou, T. Velkov, J. Li, and X. Xiao. Antimicrob Agents Chemother., 2015, 59, 579585

180 L. Dalfino, F. Puntillo, M. J. Ondok, A. Mosca, R. Monno, S. Coppolecchia, M. L. Spada, F. Bruno and N. Brienza, Clin Infect Dis. 2015, 61, 1771-7

181 R. Sirijatuphat, S. Limmahakhun, V. Sirivatanauksorn, R. L. Nation, J. Li and V. Thamlikitkul. Antimicrob Agents Chemother. 2015, 59, 3224-3232

182 M. S. Oliveira, G. V. Prado, S. F. Costa, R. S. Grinbaum and A. S. Levin AS. Polymyxin B and colistimethate are comparable as to efficacy and renal toxicity. Diagn. Microbiol. Infect. Dis. 2009, 65,431-4

183 K. Z. Vardakas and M.E. Falagas. Int. J. Antimicrob. Agents, 2017, 49, 233-238

184 K. Phe, Y. Lee, P. M. McDaneld, N. Prasad, T. Yin, D. A. Figueroa, W. L. Musick, J. M. Cottreau, M. Hu and V. H. Tam Antimicrob. Agents Chemother. 2014, 58, 2740-6.

185 A. P. Zavascki and R. L. Nation, Antimicrob. Agents Chemother., 2017, 61, pii: e02319-16

186 S.-E. Cheah, J. Li, B.T. Tsuji, A. Forrest, J. B. Bulitta and R. L. Nation Antimicrob. Agents Chemother. 2016 vol. 60 no. 7 3921-3933

187 MdF Fernandes Vattimo, M. Watanabe, C. Dezoti da Fonseca , L. Barros de Moura Neiva , E. Andrade Pessoa and F. Teixeira Borges, Plos One 2016, 11, e0161057

188 L. S. McCoy, K. D. Roberts, R. L. Nation, P. E. Thompson, T. Velkov, J. Li, and Y. Tor, Chembiochem, 2013, 14, 2083-2086.

189 T. Suzuki, H. Yamaguchi, J. Ogura, M. Kobayashi, T. Yamada and K. Iseki, Antimicrob. Agents Chemother., 2013, 57, 6319-6324.

190 S. K. Moestrup, S. Cui, H. Vorum, C. Bregengård, S. E. Bjørn, K. Norris, J. Gliemann and E. I. Christensen, J. Clin. Invest. 1995, 96, 1404-1413.

191 J. M. Lopez-Novoa, Y. Quiros, L. Vicente, A. I. Morales, and F. J. Lopez-Hernandez, Kidney Int. 2011, 79, 33-45.

192 P. Manchandani, J. Zhou, J. T. Babic, K. R. Ledesma, L. D. Truong and V. H. Tam, Antimicrob. Agents Chemother. 2017 AAC-02391. Posted Online 17 January 2017

193 A. P. Zavascki, L. Z. Goldani, G. Y. Cao, S. V. Superti, L. Lutz , A. L. Barth, F. Ramos, M. M. Boniatti, R. L. Nation and J. Li, Clin. Infect. Dis. 2008, 47, 1298-1304

194 M. A. K. Azad, b. A. Finnin, A. Poudyal, K. Davis, J. Li, p. A. Hill, R. L. Nation, T. Velkov and J. Li, Antimicrob. Agents Chemother., 2013, 57, 4329-4335

195 J. Li, K. Coulthard, R. Milne, R.L. Nation, S. Conway, D. Peckham, C. Etheringtonand J. Turnidge, J. Antimicrob. Chemother., 2003,52, 987-992.

196 Li, J.; Milne, R.W.; Nation, R.L.; Turnidge, J.D.; Smeaton, T.C.; Coulthard, K. J. Antimicrob. Chemother., 2004, 53, 837840

197 N. D. Keirstead, M. P. Wagoner, P. Bentley, M. Blais, C. Brown, L. Cheatham, P. Ciaccio, Y. Dragan, D. Ferguson, J. Fikes, M. Galvin, A. Gupta, M. Hale, N. Johnson, W. Luo, F. McGrath, M. Pietras, S. Price, A. G. Sathe, j. C. Sasaki, D. Snow , R. L. Walsky and G. Kern, Toxicol Sci. 2014, 137, 278-91

198 A. Nilsson, R. J. A. Goodwin, J. G. Swales, R. Gallagher, H. Shankaran, A. Sathe, S. Pradeepan, A.Xue, N. Keirstead, J. C. Sasaki, P. E. Andren and A. Gupta, Chem. Res. Toxicol. 2015, 28, 1823-1830

199 T. Velkov, B. Yun, E. K. Schneider, M. A. K. Azad, O. Dolezal, F. C. Morris, R. L. Nation, J. Wang, K. Chen, H. H. Yu, L. Wang, P. E. Thompson, K. D. Roberts and J. Li, ACS Infectious Diseases 2016, 2, 341-351

200 M. A. K. Azad, K. D. Roberts, H. H. Yu, B. Lu, A. V. Schofield, S. A. James, D. L. Howard, R. L. Nation, K. Rogers, M. D. de Jonge, P. E. Thompson, J. Fu, T. Velkov, and J. Li, Anal. Chem. 2015, 87, 1590-1595. 
201 B. Yun, M. A. K. Azad, C. J. Nowell, , R. L. Nation, P. E. Thompson, K. D. Roberts, T. Velkov and J. Li, Antimicrob. Agents Chemother. 2015, 59, 7489-7496.

202 B. Yun, M. A. K. Azad, J. Wang, R. L. Nation, P. E. Thompson, K. D. Roberts, T. Velkov and J. Li, J. Antimicrob. Chemother. 2015, 70, 827-829.

203 E. A Lock and C. J. Reed, Toxicologic Pathology, 1998, 26, $18-25$

204 D. Burt, S. J. Crowell, D. C. Ackley, T. V. Magee and J. Aubrecht, Drug Chemical Toxicol., 2014, 37, 204-212,

205 J. V. Bonventre, V. S. Vaidya, R. Schmouder R, P. Feig and F. Dieterle, Nat Biotechnol 2010, 28, 436-440.

206 J. Alsina , F. Rabanal , E. Giralt and F. Albericio, Tetrahedron Lett., 1994, 35, 9633

207 J. Alsina , F. Rabanal, C. Chiva, E. Giralt and F. Albericio, Tetrahedron 1998, 54, 10125

208 S. Chihara, M. Yahata, T. Tobita and Y. Koyama, Agric. Biol. Chem. 1974, 38, 521-529

209 S. Chihara, T. Tobita, M. Yahata, A. Ito and Y. Koyama, Agric. Biol. Chem. 37, 2455-63 (1973).

210 T. Suzuki, K. Hayashi and K. Fujikawa, J. Biochem, 1963, 54, 412

211 Y. Kimura, H. Matsunaga, M. Vaara, J Antibiot (Tokyo). 1992, 45, 742-9

212 R. A. Leese patent aplication W02010075416

213 J. Quale, N. Shah, P. Kelly, E. Babu, M. Backer, G. RosasGarcia, J. Salamera, A. George, S. Bratu, D. Landman, Microb. Drug Resist. 2012, 18, 132-136

214 T. V. Magee, M. F. Brown, J. T. Starr, D. C. Ackley et al.. J. Med. Chem., 2013, 56, 5079-5093

215 T. Velkov, K. D. Roberts, R. L. Nation, J. Wang, P. E. Thompson, and J. Li, ACS Chem. Biol. 2014, 9, 1172-1177

$2162^{\text {nd }}$ International Conference on Polymyxins, September, 22-24, 2015 San Diego, California, USA http://www.isap.org/images/polymyxinconference2015/Day-2-slides-session-8-2.pdf

217 A. Gallardo-Godoy, C. Muldoon, B. Becker, A. G. Elliott, L. H. Lash, J. X. Huang, J. X. Huang, M. S. Butler, R. Pelingon, A. M. Kavanagh, S. Ramu, W. Phetsang, M. A. T. Blaskovich and M. A. Cooper, J. Medicinal Chem., 2016, 59, 1068-1077

218 M. Vaara, J. Fox, G. Loidl, O. Siikanen, J. Apajalahti, F. Hansen, N. Frimodt-Møller, J. Nagai, M. Takano and T. Vaara Antimicrob. Agents Chemother. 2008, 52, 3229-36.

219 M. Vaara, H. S. Sader, P. R. Rhomberg, R. N. Jones and T. Vaara, J. Antimicrob Chemother. 2013, 68, 636-9.

220 M. Vaara, O. Siikanen, J. Apajalahti, N. Frimodt-Møller, and T. Vaara, J. Antimicrob Chemother. 2010, 65, 942-5

221 M. Vaara, Microbiol. Rev. 1992, 56, 395-411.

222 M. Vaara and T. Vaara, patent application WO2016/113470

223 C. Vingsbo Lundberg, T. Vaara, N. Frimodt-M $\varnothing$ ller and M Vaara, J. Antimicrob Chemother. 2010, 65, 981-5.

224 M. Vaara, T. Vaara and J. M. Tyrrell, Peptides, 2017, 91, 812

225 D. Erickson, Start Up, 2015 (online journal) https://sperotherapeutics.com/wpcontent/uploads/2016/02 /StartUP_Spero_Therapeutics_SU1512.pdf https://scrip.phärmamedtechbi.com/SC092696/SperoTherapeutics--Remodeling-Antibiotics

226 S. Coleman, M. Bleavins, T. Lister, M. Vaara, and T. R. Jr Parr, The Assessment of SPR741 for Nephrotoxicity in Cynomolgus Monkeys and Sprague-Dawley rats. Poster 523 (ASM Microbe, Boston, MA, USA, 2016).

227 Spero press release:

https://sperotherapeutics.com/news/press-releases/sperotherapeutics-initiates-first-clinical-study-lead-potentiator- candidate-treatment-multidrug-resistant-gram-negativeinfections/

228 P. Brown, M. Dawson, M. Simonovic, S. Boakes and E. Duperchy, patent application WO2014/188178

229 P. Brown, M. Dawson, M. Simonovic, S. Boakes and E. Duperchy, S. J. Stanway, A. Wilson and S. F. Moss, patent application WO2015/135976

230 H. O'Dowd, B. Kim, P. Margolis, W. Wang, C. Wu, S. L. Lopez and J. Blais, Tetrahedron Lett. 2007, 48, 2003-2005.

231 P. Brown, S. Boakes, E. Duperchy, M. Simonovic, O. Abdulle, N. Divall, S. J. Stanway, A. Wilson, S. F. Moss and M. J. Dawson. Poster F-739, 55th Interscience Conference on Antimicrobial Agents and Chemotherapy (San Diego, CA, USA, 2015).

232 N. P. Wiederhold, E. Duperchy, P. Brown, L. J. Payne and M. J. Dawson Poster F-734, 55th Interscience Conference on Antimicrobial Agents and Chemotherapy (San Diego, CA, USA, 2015).

233 S. Boakes, J. H. Jorgensen, S. Boakes, M. S. Collins, M. McElmeel, M. T. Cushion, T. F. Patterson, Poster F-735, 55th Interscience Conference on Antimicrobial Agents and Chemotherapy (San Diego, CA, USA, 2015)

234 Spero press release 2017

https://sperotherapeutics.com/news/press-releases/sperotherapeutics-acquires-next-generation-antibacterialcandidates-pro-bono-bio-treatment-multidrug-resistantgram-negative-infections/

235 N. Bodor and P. Buchwald, Med. Res. Rev. 2000, 20, 58101

236 M. F. Gordeev, J. Liu, X. Wang, Z. Yuan, patent aplication WO 2016/100578

237 F. Rabanal, patent application PCT/EP2016/079066

238 D. C Hobbs, patent US 3,450,687, 1969

239 L. H. Lash, Toxicol. Appl. Pharmacol. 2005, 204, 329- 342

240 J. W. Lohr, G. R. Willsky and M. A. Acara. Pharmacol. Rev. 1998, 50, 107-142

241 L. A. Bass, M. V. Lanahan, J. R. Duncan, J. L. Erion, A Srinivasan, M. A. Schmidt and C. J. Anderson, Bioconjugate Chem., 1998, 9, 192-200

242 H. Rudilla, E. Fusté, Y. Cajal, F. Rabanal, T. Vinuesa and M. Viñas, Molecules, 2016, 21, 1223

243 I. Karaiskos, M. Souli, I. Galani, and H. Giamarellou, Expert Opinion on Drug Metabolism \& Toxicology, 2017, 13, http://dx.doi.org/10.1080/17425255.2017.1230200

\section{Formatting - please delete this box prior to submission}

- Graphics, including tables, will be located at the top or bottom of the column following their first citation in the text during production (unless they are equations, which appear in the flow of the text). They can be single column or double column as appropriate and require appropriate captions.

- Text is not wrapped around any of the graphics.

- During production, sufficient space will be inserted around graphics for clarity of reading; a horizontal bar will also be used to separate all inserted graphics, tables and their captions from the text:

- Please consult the Styles menu for recommended formatting for all text, including footnotes, references, tables, images and captions. 


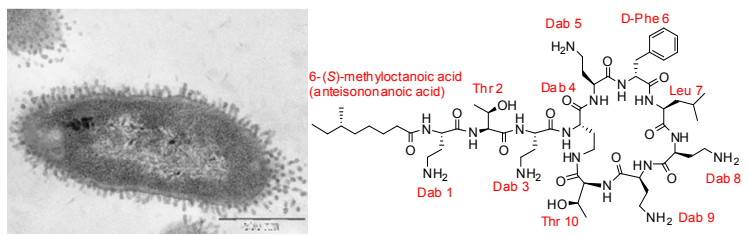

Polymyxins: background and future perspectives. Introducing metabolism and detoxification considerations into the design of new polymyxins to reduce nephrotoxicity 